\title{
Mechanics of suspended cells probed by dual optical traps in a confocal microscope
}

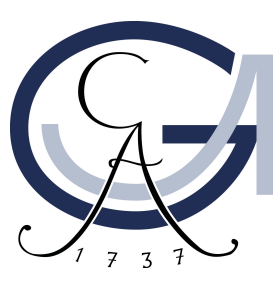

by Florian Schlosser 



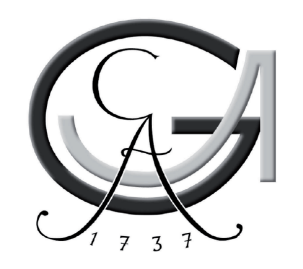

\title{
Mechanics of suspended cells probed by dual optical traps in a confocal microscope
}

\author{
Dissertation \\ for the award of the degree \\ „Doctor rerum naturalium“ \\ of the Georg-August-Universität Göttingen
}

within the doctoral program

Physics of Biological and Complex Systems

of the Göttingen Graduate School for Neurosciences, Biophysics, and

Molecular Biosciences (GGNB)

of the Georg-August University School of Science (GAUSS)

submitted by

Florian Schlosser

from Hannover, Germany

Göttingen, June $12^{\text {th }}, 2015$ 
Members of the thesis committee:

Dr. Florian Rehfeldt ( $1^{\text {st }}$ reviewer and supervisor)

Drittes Physikalisches Institut

Georg-August Universität Göttingen

Prof. Dr. Sarah Köster ( $2^{\text {nd }}$ reviewer)

Institut für Röntgenphysik

Georg-August Universität Göttingen

Prof. Dr. Christoph F. Schmidt (co-supervisor)

Drittes Physikalisches Institut

Georg-August Universität Göttingen

Prof. Dr. Andreas Janshoff

Institut für physikalische Chemie

Georg-August Universität Göttingen

Additional members of the examination committee:

Dr. Claus Heussinger

Institut für Theorethische Physik

Georg-August Universität Göttingen

Dr. Iwan Schaap

Drittes Physikalisches Institut

Georg-August Universität Göttingen

Date of the oral examination: July $15^{\text {th }}, 2015$ 


\section{Affidavit}

I herewith declare that I have produced this dissertation without the prohibited assistance of third parties and without making use of aids other than those specified; notions taken over directly or indirectly from other sources have been identified as such. This dissertation has not previously been presented in identical or similar form to any other German or foreign examination board.

The thesis work was conducted from June 2011 to May 2015 under the supervision of Dr. Florian Rehfeldt and Prof. Dr. Christoph F. Schmidt at the Third Institute of Physics - Biophysics.

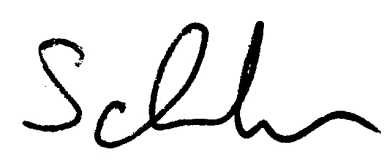

Florian Schlosser

Göttingen, June $12^{\text {th }}, 2015$ 



\section{Summary}

Cells are sensitive to mechanical cues from their environment and at the same time generate and transmit forces to their surroundings. In this thesis, we quantitatively measured forces and elastic spring constants of suspended cells. We used a dual optical trap to attach micrometer-sized fibronectin-coated beads to opposite sides of a rounded cell and detected the position fluctuations of the beads with high temporal and spatial resolution. Using a force-feedback mechanism and an acoustooptical deflector we could apply constant or oscillatory external forces to the cell. We found that the elastic response and force generation of cells are strongly governed by their acto-myosin cortex. Cell stiffness decreased substantially with both myosin inhibition by blebbistatin and serum-starvation, but not with microtubule depolymerization by nocodazole. Experiments using giant unilamellar vesicles as a model system showed that the contribution of the lipid envelope to the elastic response of cells is negligible. Force fluctuation experiments showed that cortical forces generated by non-muscle myosin II (NMM II) are present in the range from 0.1 to $10 \mathrm{~Hz}$. While blebbistatin treatment and transfer to serum-free medium strongly reduced the force fluctuations, microtubule depolymerization did not affect them.

To be able to track changes in the acto-myosin cytoskeleton, we built a novel setup incorporating a dual optical trap into a Leica SP5 X confocal microscope. We showed that it is possible to detect the force fluctuations that a LifeAct-RFP and non-muscle myosin II-GFP co-transfected cell transmits to two attached beads, and image its acto-myosin cytoskeleton at the same time for up to one hour. The observed filamentous actin network of fibroblast cells in suspension is highly diverse, ranging from cells with only a flat actin cortex to cells that have actin bundles spanning through their whole interior cytosol. We found that cardiac fibroblasts in control medium have a $40 \%$ thicker actin cortex than cardiac fibroblasts treated with $100 \mu \mathrm{M}$ blebbistatin.

We further investigated active and passive mechanical properties of cells used in engineered heart muscle. We found that primary fibroblasts originating from heart, skin and gingiva have comparable spring constants. Cardiac fibroblasts proved to have much higher force fluctuations than the other primary fibroblasts. The amplitude of those force fluctuations is even more strongly relying on force generation of myosin II motors compared to NIH 3T3 cells indicated by a roughly fifty-fold reduction after blebbistatin treatment. We could also prove that it is possible to use the dual optical trap setup to measure contraction forces and beating frequency of cardiomyocyte cells. 



\section{Table of contents}

$\begin{array}{lll}\text { Chapter } 1 & \text { Introduction } & 1\end{array}$

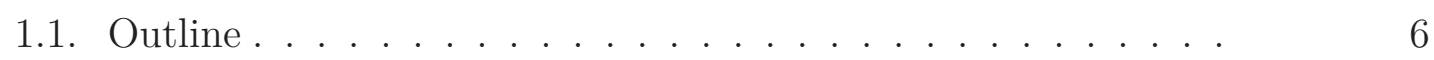

$\begin{array}{lll}\text { Chapter } 2 & \text { Materials and methods } & 7\end{array}$

2.1. Optical trapping ... . . . . . . . . . . . . . . . . . . . . 7

2.2. Force-feedback mode . . . . . . . . . . . . . . . 15

2.3. Measuring cellular force fluctuations . . . . . . . . . . . 18

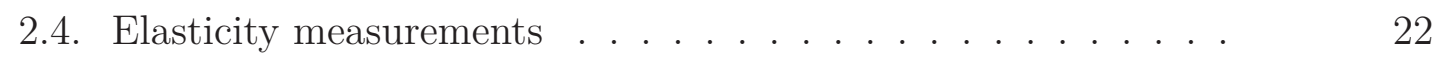

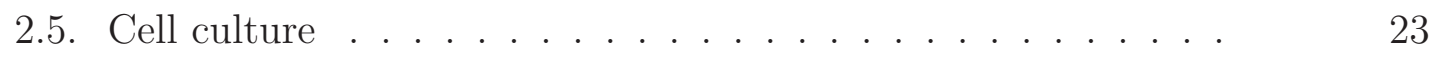

2.6. Vesicle preparation ... . . . . . . . . . . . 24

Chapter 3 Force fluctuations of suspended cells $\quad \mathbf{2 5}$

3.1. Introduction . . . . . . . . . . . . . . . . 25

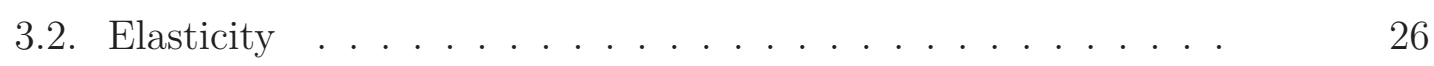

3.3. Force fluctuations . . . . . . . . . . . . . . . 28

3.4. Bead size . . . . . . . . . . . . . . . . . 34

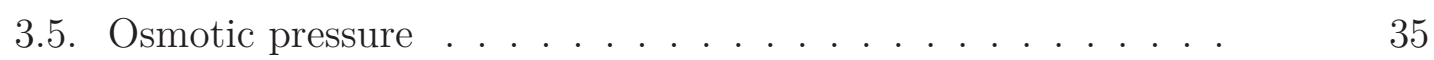

3.6. Discussion . . . . . . . . . . . . . . . . . 36

$\begin{array}{lll}\text { Chapter } 4 \text { Construction of a confocal trap } & 39\end{array}$

4.1. Confocal trap . . . . . . . . . . . . . . . . . . 40

4.2. Characterization of the trap . . . . . . . . . . . . 43

4.3. Fibroblasts in the confocal trap ............. . . . . . . . . . 46

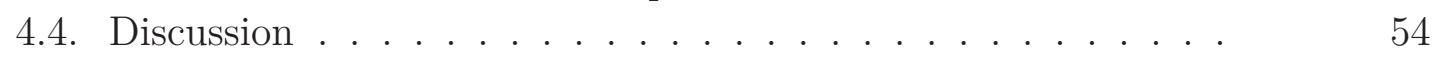

Chapter 5 Mechanical properties of cells in engineered heart $\begin{array}{ll}\text { muscle } & 57\end{array}$

5.1. Engineered heart muscle . . . . . . . . . . . . . . . 57

5.2. Force fluctuations and elasticity of primary fibroblasts . . . . 60

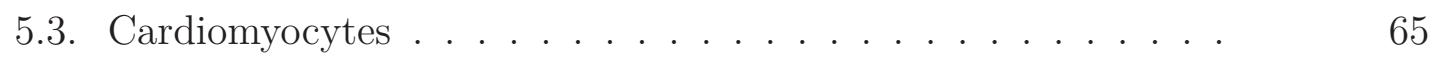

5.4. Discussion . . . . . . . . . . . . . . . . . . . 67

$\begin{array}{lll}\text { Chapter } 6 & \text { Conclusions and outlook } & 69\end{array}$ 
$\begin{array}{ll}\text { A Biochemical protocols } & 73\end{array}$

A.1. Coating of beads . . . . . . . . . . . . . . . . . . . 73

A.2. Preparation of vesicles . . . . . . . . . . . . . . . . . 74

A.3. DDS coating of coverslips . . . . . . . . . . . . 75

A.4. Fibroblast cell culture . . . . . . . . . . . . . . 75

A.5. Detachment of cardiomyocytes . . . . . . . . . . . . . 76

A.6. Procedure for extracellular microrheology . . . . . . . . . . . 77

$\begin{array}{ll}\text { List of references } & 79\end{array}$

$\begin{array}{ll}\text { Acknowledgments } & 91\end{array}$

$\begin{array}{ll}\text { Curriculum Vitae } & 93\end{array}$ 


\section{\begin{tabular}{l|l} 
& \\
Introduction & 1
\end{tabular}}

The mechanical properties of cells and their ability to sense and exert mechanical signals play a major role many functions. Change in the mechanical environment of cells or externally applied forces have been shown to alter cell migration [1], cell growth [2] or even stem cell differentiation [3]. The response of cells to external forces can be seen as a two step process. First a mechanical response [4, 5] as a deformation of the cellular structures and secondly a biochemical response [6, 7] that in the end would lead to a phenotypic change. To understand the biochemical signaling of the cell, one has to understand the mechanical response first.

The most prominent structure in the cell that have been found to play a role in mechanical stability and force generation is known as the cellular cytoskeleton (figure 1.1). The three major components of the cytoskeleton are proteins that assemble into filamentous structures and form networks that span the whole cellular interior: actin, microtubules and intermediate filaments. Furthermore there are numerous proteins that are associated with these network structures and help them to crosslink themselves, to interact with each other, to contract or expand and to link to the extracellular matrix. The network that is able to generate contractile forces in cells is the actin network interacting with myosin molecular motors as force generating elements, the so called acto-myosin network. Networks of microtubules and intermediate filaments do not generate contractile forces besides cell division, where the mitotic spindle composed of microtubules drives DNA separation.

Figure 1.1: Fluorescence image of NIH 3T3 fibroblasts adherent on fibronectin-coated glass slide. Fixed with formaldehyde and stained for actin (phalloidin-TRITC, red), microtubule (green) and nucleus (Hoechst, blue).

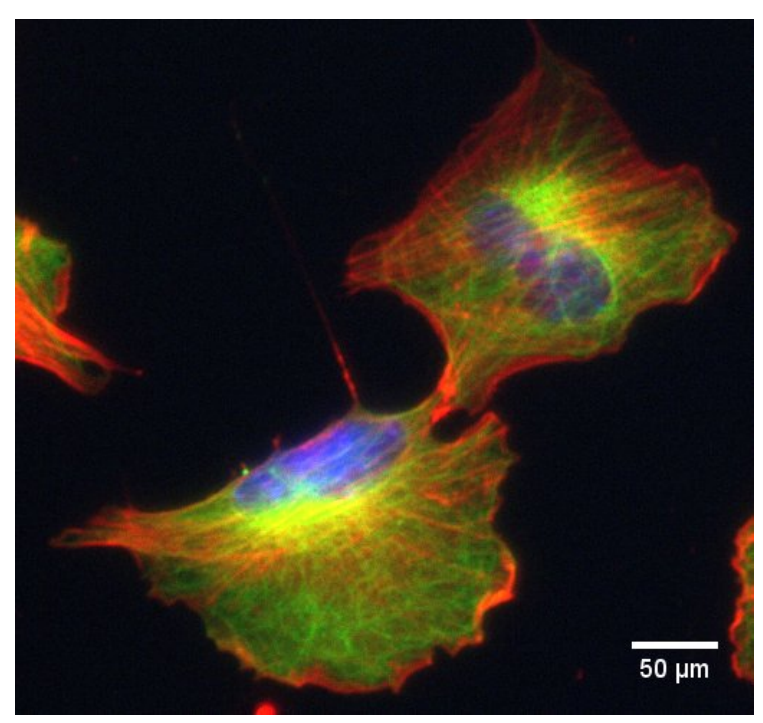




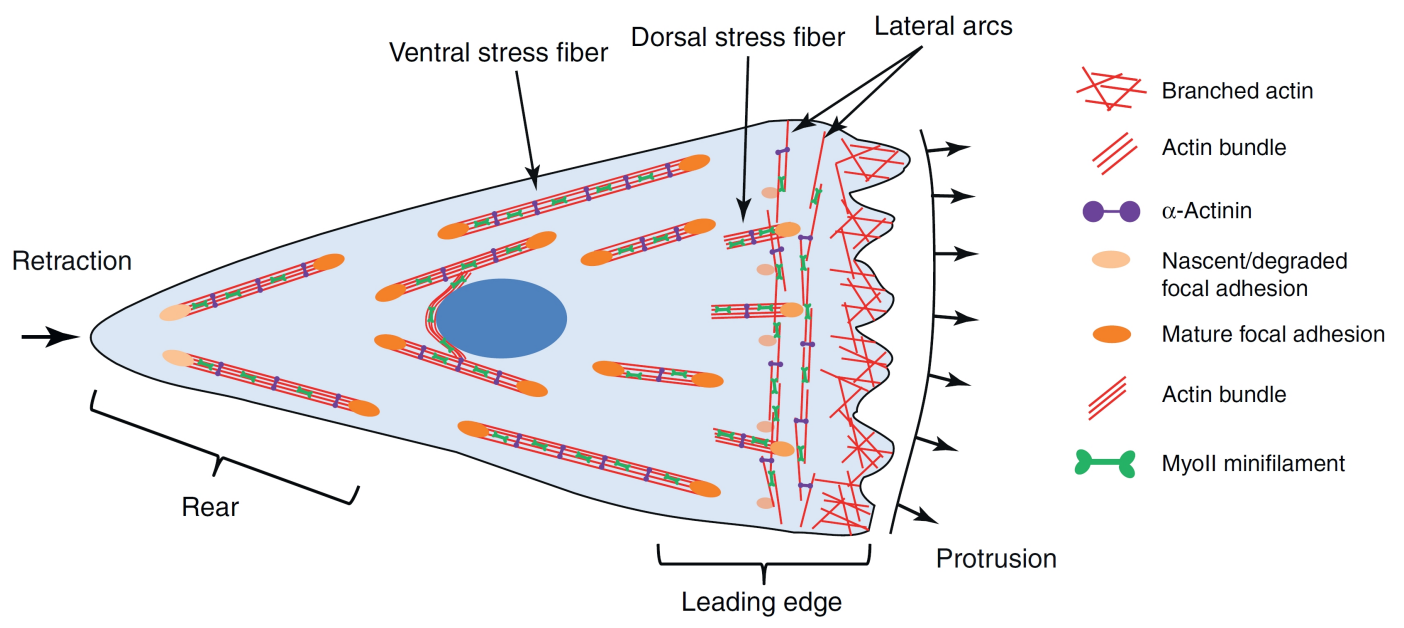

Figure 1.2: Different families of stress fibers in a migrating fibroblast on a $2 \mathrm{D}$ adhesive substrate. Adapted from [8]

In migrating cells, like fibroblasts, the acto-myosin network is the key player in all force-related actions. Actin polymerization forces drive protrusions on the leading edge to move forward. In the retracting rear end of the cells, where high contractile forces are needed, actin, non-muscle myosin II (NMM) and crosslinks bundle into so-called stress fibers [9] (figure 1.2) to destroy the adhesion contacts.

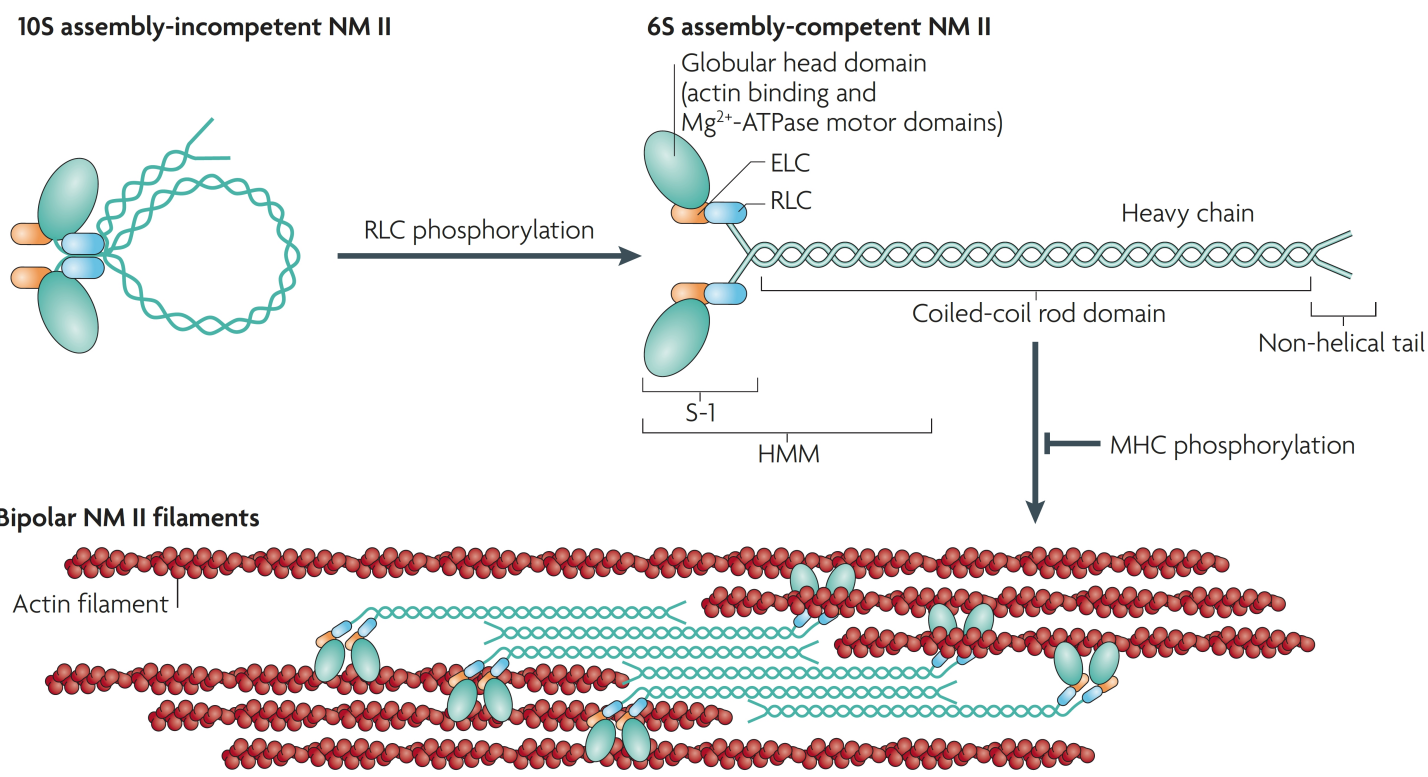

Figure 1.3: The subunit and domain structure of non-muscle myosin II, which forms a dimer. The actin binding domain is located in the head. Essential light chain (ELC) and regulatory light chain (RLC) link the head to the heavy chain. RLC phosphorylation makes the structure assembly-competent. NMM molecules assemble into bipolar filaments through interactions between their rod domains. They bind to actin filaments with their head and ATPase activity of the head enables conformational changes that move the actin filaments in an anti-parallel manner. Adapted from [10].

Generation of high contractile forces is mainly due to interactions of myosin motors with actin filaments in the stress fibers. Phosphorylated NMM proteins selfassociate into bipolar minifilaments (figure 1.3). These minifilaments are able to 


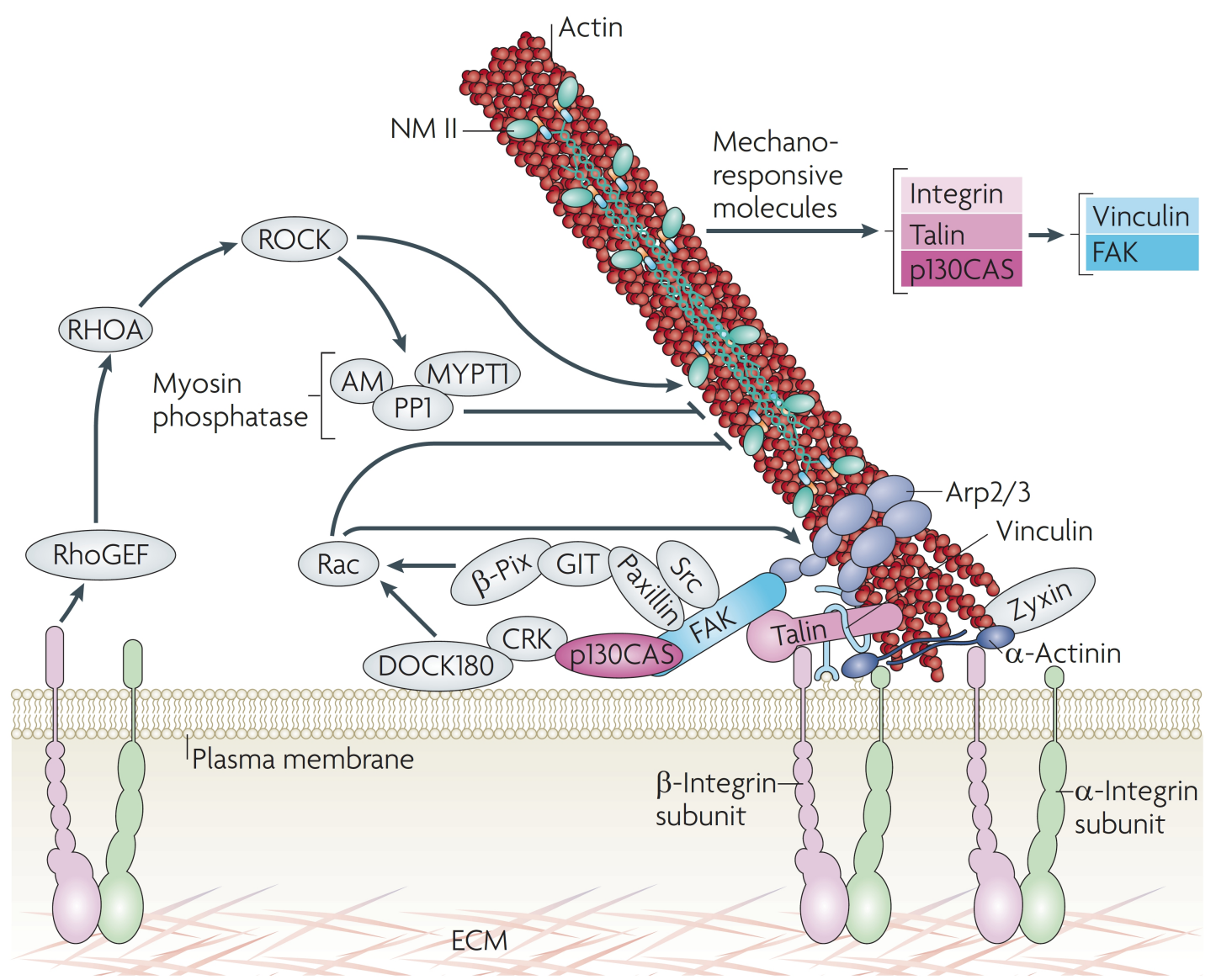

Figure 1.4: NMM in integrin-mediated adhesion. Integrins that are bound to the extracellular matrix (ECM) are linked to the actin cytoskeleton through an active linkage that is formed by multiple molecules called focal adhesion. Representative pathways and associations are shown. Adapted from [10].

crosslink actin and, by conformational changes due to ATPase activity, move along the actin filament in an anti-parallel manner sliding them towards each other. With this movement, they generate tension in the actin cytoskeleton. To transmit these forces to the surrounding, stress fibers are anchored at protein complexes where they are coupled to proteins that are embedded in the plasma membrane of the cell and can connect to the extracellular matrix (ECM) (figure 1.4). A detailed description of the role of non-muscle myosin II in the generation of contractile forces can be found in [10].

To measure cellular forces, lots of different techniques have been established including traction force microscopy $[12,13,14]$ and growing cells on elastic micropillars [15]. Various techniques to measure the mechanical properties have been established, mainly focusing of rheological measurements of cell properties ranging over different frequency ranges. A collection of methods are shown in figure 1.5 and a more detailed description of those techniques and the interpretation of rheological measurements can be found in reference [11].

However, all of these mechanisms for generation of high contractile forces and all of the described techniques have in common that they are done on cells that adhere to a flat $2 \mathrm{D}$ surface, a rather unphysiological situation. In their natural environment, 

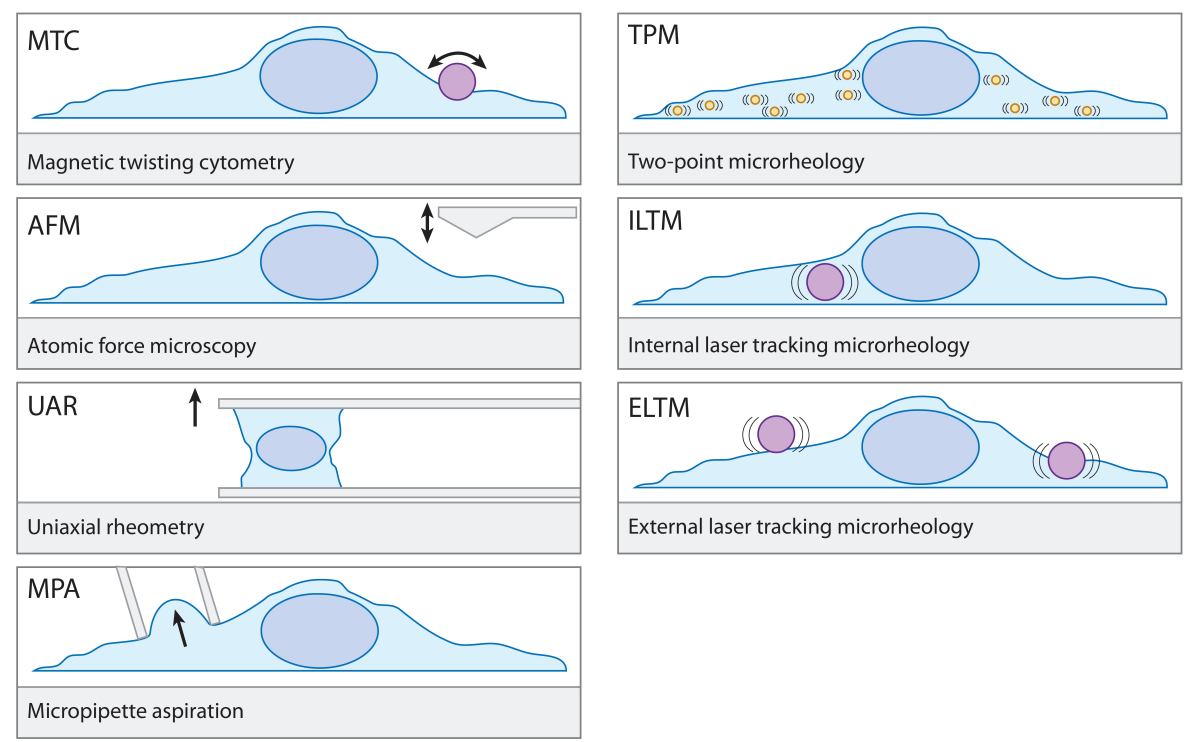

Figure 1.5: Schematic comparison of common cell rheology methods. Adapted from [11]

cells are embedded in a 3D tissue. Their nearest surroundings either consist of other cells or a viscoelastic network. One option to overcome the $2 \mathrm{D}$ limitation is presented in this thesis. We use a dual optical trap to hold cells in suspension with two attached beads (figure 1.6). This has the opportunity, in contrast to most of the described methods above, to measure cellular forces and probe the rheological properties of the cell over a wide frequency range at the same time. In this configuration, the cell

Figure 1.6: Schematic of the measurement procedure used in this thesis. Two fibronectin-coated beads are attached to opposite sides of a rounded cell. A dual optical trap is used to hold the beads in suspension and measure the displacement fluctuations that the cell transmits to the beads. (Schematic by Florian Rehfeldt).

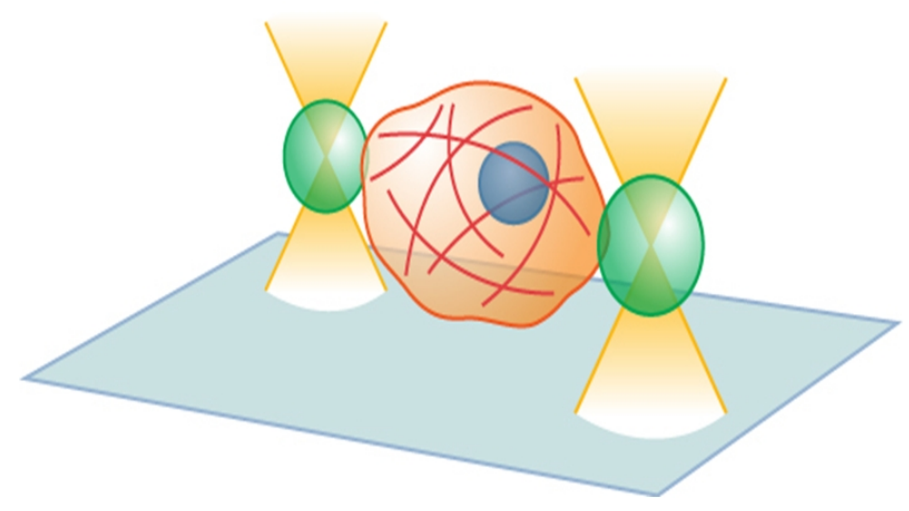

has a rounded up geometry close to its native appearance, where most of the actin is located in the cell cortex, a thin, crosslinked network lying immediately beneath the plasma membrane of the cells. The acto-myosin cortex is an essential but still poorly understood actin network. It is crucial for cells to have proper control of the mechanical properties of the cortex. The cortex is the main player determining the stiffness of the cell surface and allows it to resist mechanical stresses and opposes intracellular osmotic pressure [16]. Turnover of proteins involved in the cortex and myosin generated contraction leads to a dynamic remodeling of the network on the order of seconds. The mechanical stability on the one hand and the remodeling potential on the other hand leads to a dynamic plasticity of the cortex, which allows the cell to rapidly change its shape, move and exert forces. Figure 1.7 shows the main components of the cortex and their interplay. 
A
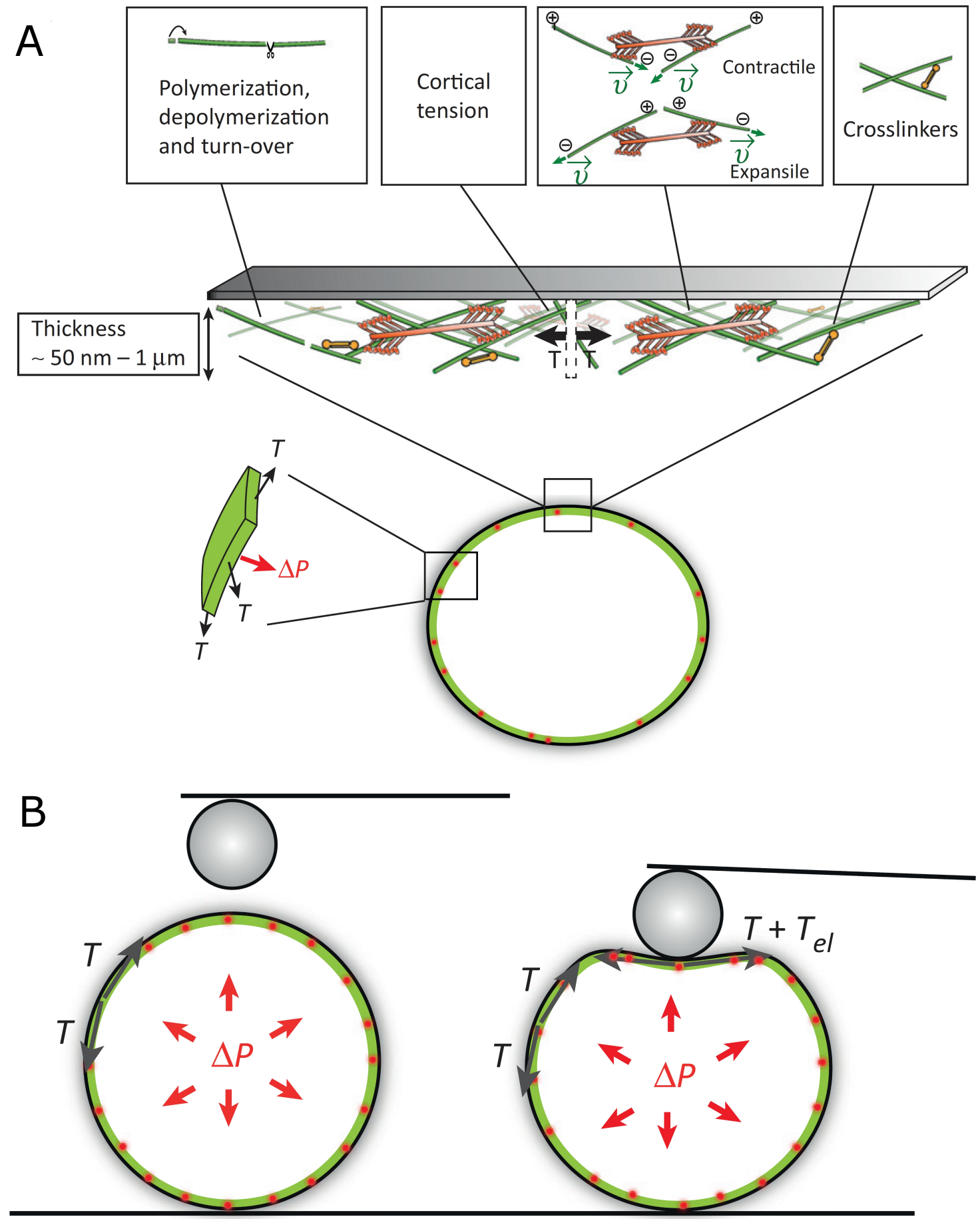

Figure 1.7: Cortex tension and composition. A) Actin filaments assemble in a thin network connected to the cell membrane. Myosin motors assemble into minifilaments and exert forces in the network, giving rise to cortical tension $T$. Due to the cell curvature, cortical tension also leads to a hydrostatic pressure $P$ in the cytoplasm. B) Schematic of an indentation experiment. The force exerted by the cell on the bead can depend both on the tension of the cortical network and on its elastic deformation. Adapted from [17] 
Non muscle myosin II is the main source of contractile forces. However, in contrast to adherent cells, myosin motors are not arranged in highly ordered stress fibers. The origin of contractile forces in the isotropic cortical network is not clear. The cell stiffness is a combination of the elastic modulus of the cortex, cortex tension and cell volume (figure 1.7B). It is also known that both networks of microtubules and intermediate filaments interact with the acto-myosin cortical network $[18,19]$. However, their contribution to active and passive mechanical properties of the cortex or their function in regulatory processes is not clear. Furthermore, the role of the interaction between the cortex and the lipid composition of the plasma membrane is poorly understood.

The data presented in this thesis will help to better understand the mechanical properties of rounded cells in suspension. It dissects contributions of different cytoskeletal filaments to force generation and elasticity of the cells and also points out differences between cells of various origins.

\subsection{Outline}

To measure the elastic properties and force generation of cells, we used a dual optical trap. An introduction into optical trapping, use and culture of the cells and the basic principles of the measurement is given in chapter 2. The force clamp, which was necessary to apply constant forces to the suspended cells, is also described in this chapter.

Chapter 3 describes the results of the optical trapping experiments. Elastic properties of the cells were investigated using external forces. Force fluctuations that the cell generates were measured in a constant position and a constant force mode. The chapter is based on the publication:

Florian Schlosser, Florian Rehfeldt, and Christoph F. Schmidt, "Force fluctuations in three-dimensional suspended fibroblasts", Philosophical Transactions B of the Royal Society, 370, 20140028, 2014.

In chapter 4 , the construction of a confocal trap coupled into a confocal microscope is explained and characterized. We use this setup to measure cellular force fluctuations and image the acto-myosin cytoskeleton of the suspended cell at the same time. Preliminary experiments investigating the assembly of focal adhesions in the 3D suspended cells are presented.

Chapter 5 is about the investigation of mechanical properties of cells used in engineered heart muscle. After an introduction into assembly and use of engineered heart muscle, the mechanical properties of primary fibroblasts originating from different tissue are analyzed and compared. In the end, measurements of beating cardiomyocytes that were suspended in the optical trap are presented. 


\title{
Materials and methods 2
}

\author{
Optical trapping $\mid 2.1$
}

\begin{tabular}{l|l} 
Trapping theory & 2.1 .1
\end{tabular}

Since the discovery of optical traps almost 30 years ago, when Arthur Ashkin and co-workers found that dielectric objects could be trapped and manipulated by laser light $[20,21,22]$, they have become an important tool in biophysics. They can be used to manipulate the right range of particle size (between a few nanometers and up to 10 micrometers) and can achieve force sensitivity up to 100 piconewtons that is relevant for biological systems, thus allowing physical characterizations of these systems. In most experiments, the probe particles were attached specifically or unspecifically to biological proteins of interest. Then either the force that the protein is generating can be measured or an external force can be applied to the protein. The principle of optical trapping is based on momentum transfer from laser light to the trapped object. Photons, like all moving particles, carry momentum $p$ which can be transferred to dielectric particles upon reflection or refraction. This radiation pressure is negligible for macroscopic particles but plays an important role in the $\mu \mathrm{m}$ range, where most of the biological relevant objects are present. Forces of the refracted light have to be higher than or at least balance forces of the scattered light to create stable trapping.

\section{Ray-optics regime}

In the geometric-optics limit, where Mie-scattering dominates $(d \gg \lambda)$, microspheres can be seen as small lenses. The momentum transfer of light refracted by these spheres of a refractive index $n$ higher than that of the surrounding medium $n_{m}$ can be calculated. The momentum change of the light due to the refraction is generating an opposing momentum transfer on the sphere according to Newton's second law. The gradient of a Gaussian profile of a laser beam is therefore always pushing the sphere towards regions of higher intensity, i.e. the force is directed to the laser focus. Stable trapping is possible if the gradient force from refracted rays is greater 


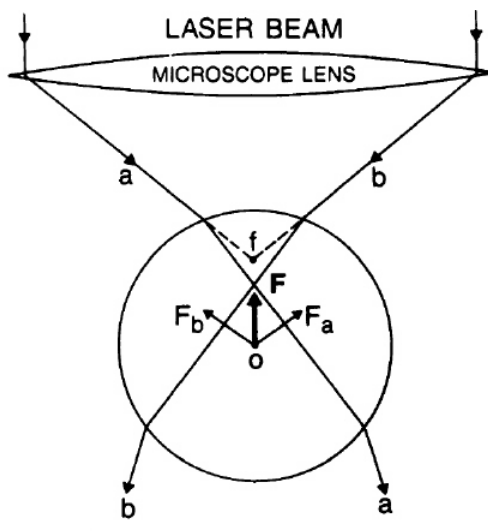

A

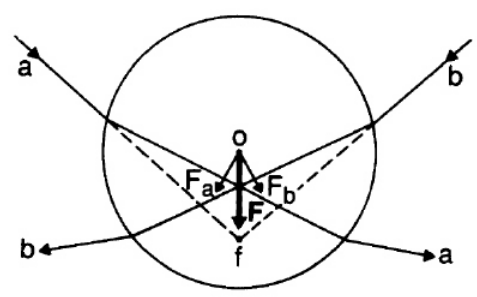

B

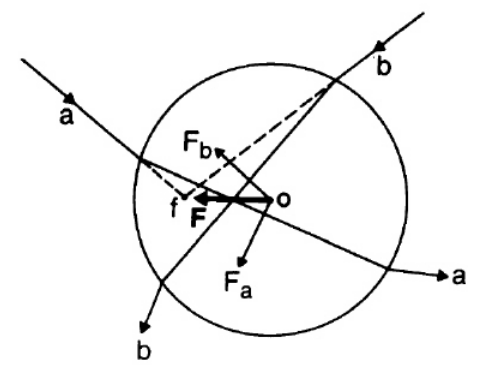

C

Figure 2.1: Diagram of refractive forces in ray-optics regime for typical beams a and $b$ and their resulting forces $F_{A}$ and $F_{B}$ for a sphere above $(\mathbf{A})$ and below $(\mathbf{B})$ the focal point $f$ of an microscope objective. (C) is showing a sphere displaced to the right of the beam focus. The resulting force $F$ is always pointing towards the focus. Figure adapted from [23]

than the scattering force. A description of the force on a sphere above, below and laterally displaced from the laser focus is shown in figure 2.1. Ashkin quantitatively calculated the trapping force in [23] with the Fresnel coefficients for reflection and transmission $R$ and $T$ including internal reflections in the sphere and polarization effects. The scattering force $F_{\text {scat }}$ and gradient force $F_{\text {grad }}$ are

$$
\begin{aligned}
\hat{F}_{\text {scat }} & =\frac{n_{m} P}{c}\left[1+R \cos (2 \Theta)-T^{2} \frac{\cos (2 \Theta-2 \Phi)+R \cos (2 \Theta)}{1+R^{2}+2 R \cos (2 \Phi)}\right] \hat{k} \\
& \equiv \frac{n_{1} P Q_{s}}{c} \hat{k}
\end{aligned}
$$

and

$$
\begin{aligned}
\hat{F}_{\text {grad }} & =\frac{n_{m} P}{c}\left[R \sin (2 \Theta)-T^{2} \frac{\sin (2 \Theta-2 \Phi)+r \sin (2 \Theta)}{1+R^{2}+2 R \cos (2 \Phi)}\right] \hat{i} \\
& \equiv \frac{n_{1} P Q_{g}}{c} \hat{i}
\end{aligned}
$$

with $P$ the power of the single light ray, $c$ as the speed of light, $\Theta$ and $\Phi$ angle of incidence and refraction and $\hat{k}$ and $\hat{i}$ as unit vectors parallel and perpendicular to the direction of the incident ray. $Q_{s}$ and $Q_{g}$ are the quality factors of the scattering and gradient force. Adding both forces and integrating over all rays leads to the total force exerted on the particle. The integration is shown for a variety of beam profiles in [23]. To get stable trapping, one needs to increase the gradient force over the scattering force. One can see that a maximum gradient force with respect to the scattering force is obtained for higher angle rays $\left(\approx 60^{\circ}\right)$. One method to increase the trapping force uses overfilling the objectives back aperture and therefore relatively adding more high power rays at large angles. Alternatively, one can use "donut" shaped laser profiles [24], or blocking the center beam with obstructions or phase mask [25]. 


\section{Rayleigh regime}

In the regime for particles much smaller than the laser wavelength $(d \ll \lambda)$ the electric dipole moment induced by the laser light is considered. Again, the force can be decomposed into a scattering and a gradient component. The scattering force is given by

$$
F_{\text {scat }}=\frac{n_{m}}{c}\langle S\rangle \sigma
$$

where

$$
\sigma=\frac{8}{3} \pi(k r)^{4} r^{2}\left(\frac{m^{2}-1}{m^{2}+2}\right)^{2}
$$

is the effective scattering cross section of the particle with radius $r, S=E \times B$ is the Poynting vector, $n$ is the refractive index of the particle, $n_{m}$ is the refractive index of the surrounding medium, $k=2 \pi n_{m} / \lambda$ is the wave number of the incident light and $m=n / n_{m}$ is the relative index. The scattering force again points in the direction of light propagation and is dependent on the energy flux. The gradient force is given by

$$
F_{\text {grad }}=\frac{\alpha}{2} \nabla\left\langle E^{2}\right\rangle
$$

where

$$
\alpha=n_{m}^{2} r^{3}\left(\frac{m^{2}-1}{m^{2}+2}\right)^{2}
$$

is the polarizability of the particle is the Lorentz force acting on the particle and is directed towards the intensity maximum of the trap. Again, stable trapping is achieved by maximizing the gradient force in relation to the scattering force, which means increasing the polarizability of the particle or increasing the energy gradient, therefore one can define a Q-factor, which is defined as

$$
Q=\frac{F_{\text {grad }}}{F_{\text {scat }}}=\frac{3 \sqrt{3}}{64 \pi^{5}} \frac{n_{2}^{2}}{\left(\frac{m^{2}-1}{m^{2}+2}\right)} \frac{\lambda^{5}}{r^{3} \omega_{0}^{2}}
$$

with

$$
\omega_{0}=\frac{\lambda}{\pi N A}
$$

the width of a Gaussian beam. One can see that a high numerical aperture $N A$ is needed for table optical trapping with $Q \gg 1$. One can also see that the Q-factor scales with $1 / r^{3}$, which makes the trap more stable for small particles.

In the experimental reality, however, normally particles with a diameter of the order of a micron and laser wavelengths with $\lambda=1064 \mathrm{~nm}$ are used, so $r \approx \lambda$. In this intermediate case, diffraction effects need to be taken into account and a full calculation of the momentum transfer from the laser light to the trapped object 
needs to be considered. Studies that take all these effects into account have started to appear in the last years, more details can be found in [26, 27, 28, 29].

\subsubsection{Back-focal plane interferometry}

One of the outstanding properties of optical traps is the ability to not only trap and move a particle but also to measure the position of the particle relative to the trapping laser focus. The most convenient way to measure forces is an interferometric approach. Forces that are exerted on the particle can be recorded with very high spatial (sub nm) and temporal $(100 \mathrm{kHz})$ resolution. The laser light which is used to trap a particle is deflected, a necessary property to create a stable trapping regime. This means that trapping and deflection of the beam are dependent on each other. The interferometric pattern, which arises from a superposition of the trapping laser and the part that is scattered by the trapped particle can be used in back-focal plane detection to acquire the position of the trapped particle. Gittes and Schmidt derived a formula for the first order angular far field distribution of intensity for a Rayleigh particle that is laterally displaced from the focus of a Gaussian beam perpendicular to the optical axis by a distance $x$ to be [30]:

$$
\frac{I(x)-I(0)}{I_{t o t}}=\frac{2 k^{3} \alpha}{\pi r^{2}} \exp \left(-\frac{x^{2}}{\omega_{0}^{2}}\right) \cdot \sin (k x \sin \theta \cos \phi) \exp \left(-k^{2} \omega_{0}^{2} \theta^{2} / 4\right)
$$

with intensity $I$ under the angles $\theta$ and $\phi, \omega_{0}$ the width of the Gaussian beam, $k$ the wave vector and $\alpha$ the polarizability. This calculation was expanded in [31] to include the axial direction of the trap, therefore allowing positional detection in three dimensions.

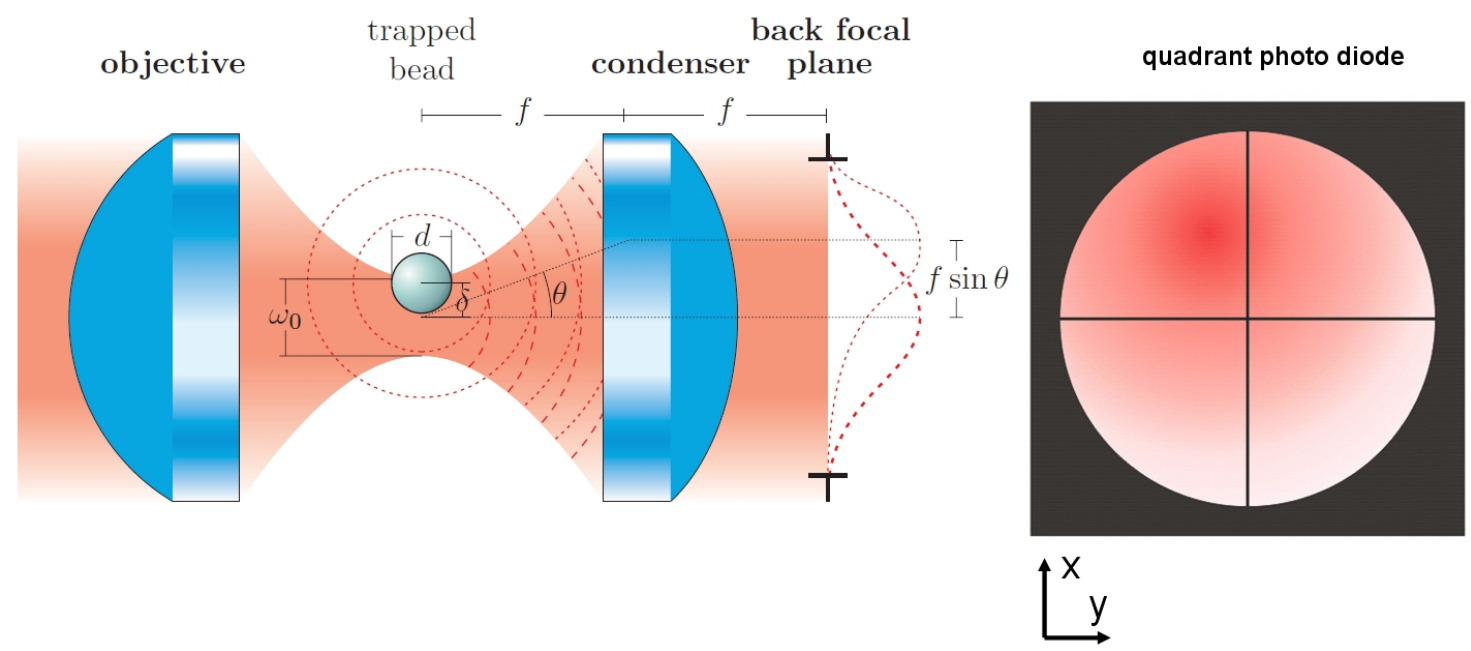

Figure 2.2: Back focal plane interferometry. Scattered and reflected light from a lateral displaced particle with diameter $d$ from the trap center by distance $\delta$ is collected by the condenser. This leads to a shift of the interference peak of $f \sin \Theta$ in the back-focal plane. Adapted from [30]. 
In the experimental setup, a condenser collects the light of the trapping laser after it passes the sample. The angular distribution of the light is translated into a spatial distribution of the intensity in the back focal plane (BFP) of the condenser (figure 2.2). This interference pattern is only dependent on the relative position of the trapped particle in the laser focus. The interference pattern in the BFP is imaged onto a quadrant photo diode (QPD) aligned with the $\mathrm{x}$ - and y-axis. The signals from the quadrants are summed pairwise and the differential signal for the $\mathrm{x}$ - and y-directions are calculated and normalized by the sum signal of the whole QPD. The detector response of the QPD is given by

$$
\Delta I=\frac{I_{+}-I_{-}}{I_{+}+I_{-}}=\frac{16 k \alpha}{\sqrt{\pi} \omega_{0}^{2}} \exp \left(-2 x^{2} / \omega_{0}^{2}\right) \int_{0}^{x / \omega_{0}} \exp \left(y^{2}\right) d y
$$

The last part is Dawson's integral and can be calculated numerically [32]. For small displacements $(\approx \pm 200 \mathrm{~nm})$, the QPD response can be approximated to be linear without crosstalk between the $\mathrm{x}$ - and $\mathrm{y}$-channels.

Calibration of optical traps

To use the optical trap for force measurements, it has to be calibrated. One can use the influence of Brownian motion on a particle with a known size in a fluid with known viscosity. Since the movement of a particle in an optical trap is not free, one needs to combine the thermal and optical force and the Langevin equation as the equation of motion for a small particle in a harmonic potential is as follows:

$$
F(t)=m \frac{d^{2} x(t)}{d t^{2}}+\gamma \frac{d x(t)}{d t}+k x(t)
$$

Here, $F(t)$ is the random thermal force which adds up to zero over time, $\gamma$ is the friction coefficient of the medium and $m$ the mass of the particle. Since the Reynolds number is very low for micrometer sized objects $\left(\operatorname{Re} \approx 10^{-4}\right.$ ), one can neglect inertial forces. One can then calculate the autocorrelation of this force

$$
\left\langle F(t) F\left(t+t^{\prime}\right)\right\rangle_{t}=C \cdot \delta\left(t^{\prime}\right)
$$

where $C \in \Re$ is a real constant, and the Fourier transform $\mathcal{F}$ of the autocorrelation, the power spectrum

$$
|F(\omega)|^{2}=C \cdot \mathcal{F}[\delta(t)]=C
$$

Using equation 2.13, one can get the power spectrum of the trapped particle, which is a Lorentz function [33]

$$
P S D(\omega)=\frac{1}{\tau}|x(\omega)|^{2}=\frac{C \tau^{-1} \gamma^{-2}}{\omega^{2}+(\kappa / \gamma)^{2}}=\frac{A}{\omega^{2}+\omega_{c}^{2}}
$$


With the critical frequency (or corner frequency) $\omega_{c}=\kappa / \gamma$, which splits the power spectrum in two regimes and the total duration $\tau$ of the signal $x(t)$. For $\omega \ll \omega_{c}$, the power spectrum is roughly constant

$$
P S D(\omega) \stackrel{\omega \ll \omega_{c}}{\approx} \frac{A}{\omega_{c}}=: S_{0}
$$

For frequencies $\omega \gg \omega_{c}$ the power spectrum follows $1 / \omega^{2}$ the same behavior that a freely diffusing object would have. This means that on short timescales the trapped object is not influenced by the optical trap. In figure 2.3, a typical powerspectrum of a trapped particle is shown.

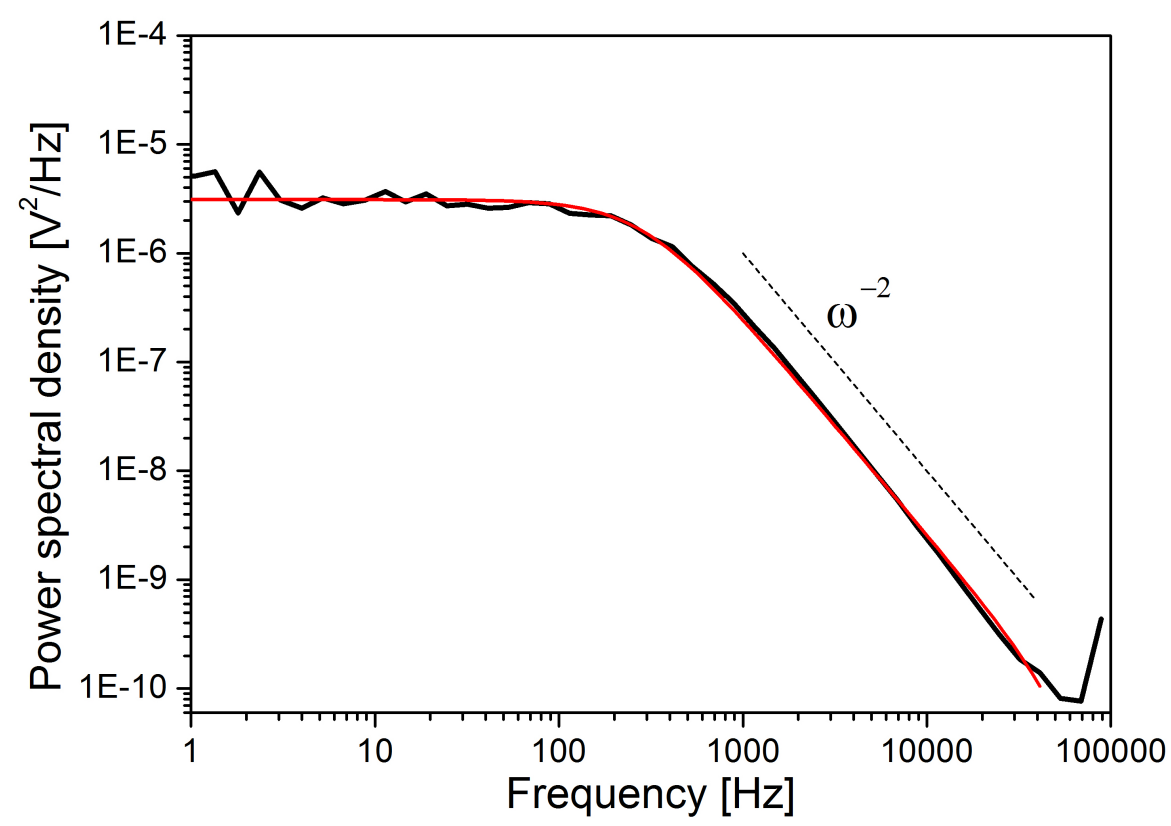

Figure 2.3: Power spectral density of a $4 \mu \mathrm{m}$ polystyrene bead. At low frequencies a plateau is visible representing the trapping potential. At higher frequencies a scaling of $\propto \omega^{-2}$ appears. This is the same behavior that a freely diffusing particle would show. In this frequency range, the particle is not affected by the optical trap. The power spectral density is fitted with a Lorentz function to obtain detector calibration and trap stiffness (red curve)

To get a value for the amplitude $A$ of the thermal force, thermal and mechanical forces are compared using Parseval's theorem. With the equipartition theorem for an harmonic potential

$$
\frac{1}{2} k_{B} T=\frac{1}{2} \kappa\left\langle x^{2}(t)\right\rangle
$$

it follows

$$
\begin{aligned}
\frac{k_{B} T}{k} & =\left\langle x^{2}(t)\right\rangle=\frac{1}{2 \pi} \int_{-\infty}^{\infty} d \omega \frac{1}{\tau}|x(\omega)|^{2}=\frac{1}{2 \pi} \int_{-\infty}^{\infty} d \omega \frac{A}{\omega^{2}+\omega_{c}^{2}} \\
& =\frac{A}{2 \pi \omega_{c}} \int_{-\infty}^{\infty} d \xi \frac{1}{1+\xi^{2}}=\frac{A}{2 \omega_{c}}
\end{aligned}
$$


with $\xi=f / f_{c}$. With this relation, one can get an expression for the amplitude $A$ of the thermal force

$$
\begin{aligned}
|F(\omega)|^{2} & =C=A \tau \gamma^{2}=2 k_{B} T \omega_{c} \tau \gamma^{2} / \kappa=2 k_{B} T \tau \gamma \\
\Rightarrow A & =\frac{2 k_{B} T}{\gamma}=2 D
\end{aligned}
$$

Fitting the power spectrum with a Lorentz function yields values for $A$ and $\omega_{c}$. With that, and the stokes drag coefficient $\gamma=6 \pi \eta r$ one can calculate the trapping stiffness $k$

$$
\begin{aligned}
& k=\frac{2 k_{B} T \omega_{c}}{A} \\
& k=6 \pi \eta r \omega_{c}
\end{aligned}
$$

From the quadrant photo diode of the trapping setup, the displacements and power spectrum of the particles movement is gained in volts

$$
\frac{1}{\tau}\left|u_{\text {elect }}\right|^{2}=\frac{A_{V}}{\omega^{2}+\omega_{c}^{2}}
$$

which is compared to the theoretical calculated power spectrum

$$
\frac{1}{\tau}\left|u_{m e c h}\right|^{2}=\frac{2 k_{B} T}{\gamma\left(\omega^{2}+\omega_{c}^{2}\right)}
$$

The calibration factor $c$ is then calculated with $u_{\text {elec }}[V] \cdot c[m / V]=u_{\text {mech }}[m]$ and yields

$$
c=\sqrt{\frac{2 k_{B} T}{6 \pi \eta r A_{V}}}
$$

Dual optical trap setup

The optical trap used for most of the experiments is integrated in a custom-built inverted microscope based on the setup first described in [34]. In short, a nearinfrared laser is polarized and split up in a parallel and a perpendicular polarized beam and focused into an objective to create two individually steerable traps. A sketch of the setup is shown in figure 2.4

The $1064 \mathrm{~nm}$ wavelength laser (Compass, Nd:IVO 4 , 4W, Coherent Inc., Santa Clara, CA, USA) is protected against back reflections by an optical isolator (IO5-1064-VHP, Thorlabs, Newton, NJ, USA). To achieve a slight overfilling of the objective, the beam is expanded by a 3x beam expander (Qioptic Photonics $\mathrm{GmbH}$ \& Co KG, München, Germany) to a size of approx. $4 \mathrm{~mm}$. For stability reasons, the laser is used at medium to high intensities. For adjusting the trapping power in the sample plane without changing the laser power, a combination of a motor driven 


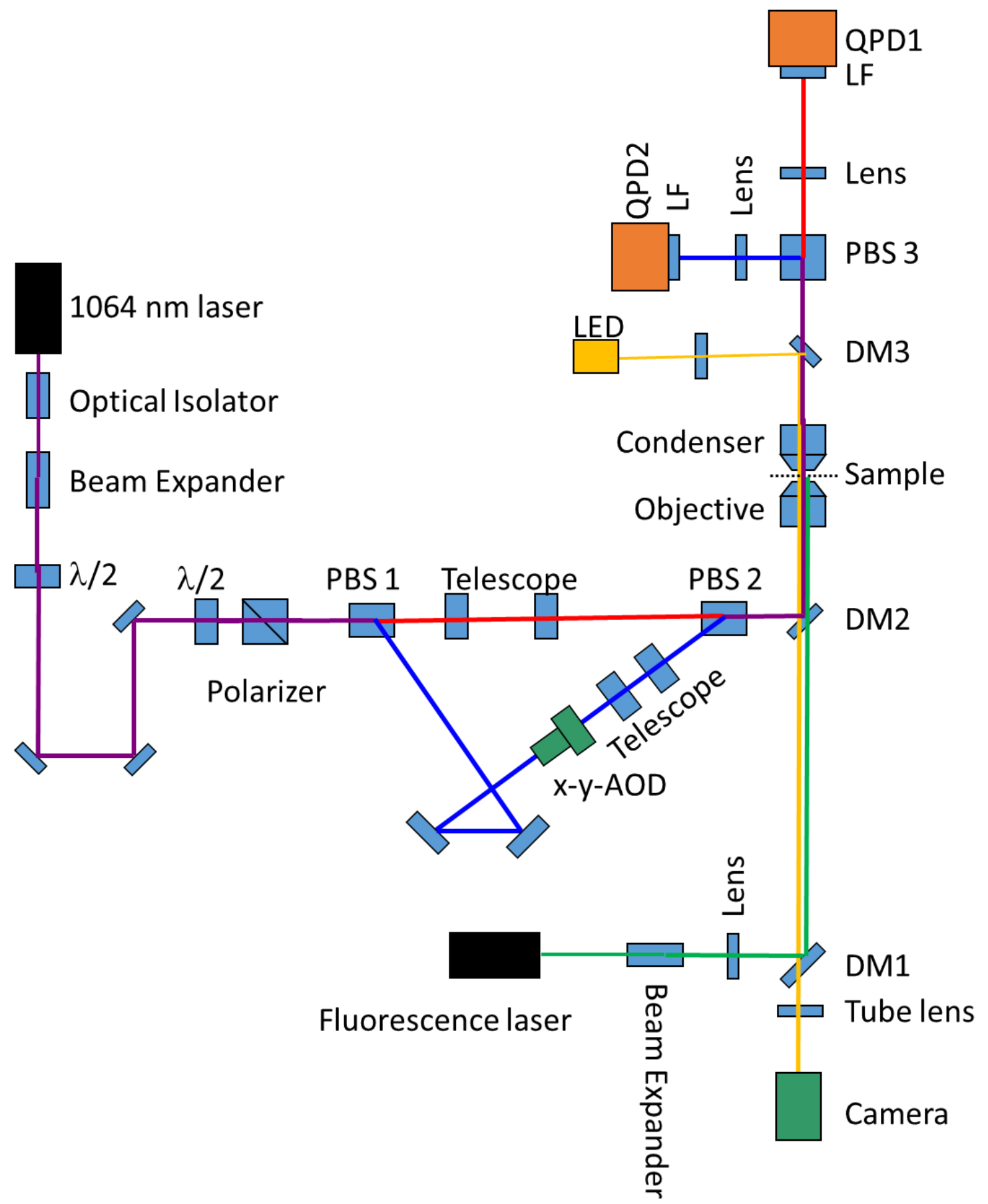

Figure 2.4: Schematic of the setup. A $1064 \mathrm{~nm}$ laser is split up into two beams by polarization and focused into a microscope to create an optical trap. Both beams are collected by a condenser and split up again on two individual QPDs for back-focal plane detection. Both beams can be steered individually by a telescope lens pair. One beam has also the option of fast steering by an AOD. The sample is illuminated by an LED and imaged by a camera. PBS:polarizing beam splitter, DM: dichroic mirror, LF: 1064 nm laser line filter. 
half-wave plate (PRM1Z8, Thorlabs, Newton, NJ, USA) and a polarizer is used. A Glan-Laser polarizing beamsplitter splits the beam in two orthogonal polarized beams (called the direct and the indirect trap). Both beams pass a 1:1 telescope lens pair which allow for individual positioning of the two traps in the specimen plane. In one of the beam paths (the indirect trap), an acousto-optical deflector (AOD) is used for fast beam steering (DTD276HB6, IntraAction, Corp., Bellwood, IL, USA). Both beams are recombined with a second Glan-Laser polarizer and coupled into the microscope with a dichroic mirror. The laser beam is focused into the sample with a 100x oil immersion objective (Neofluar, Carl Zeiss MicroImaging GmbH, Jena, Germany). The sample is mounted on a 3D translational stage which is steerable in $\mathrm{x}-\mathrm{y}$ direction with micrometer screws and in $\mathrm{z}$-direction with a stepper motor. Additional precision steering is possible with a piezo driven stage (MCL01084, Mad City Labs, Madison, Wi, USA). After passing the sample, the laser light is collected by an 1.4 oil immersion condenser (Carl Zeiss MicroImaging GmbH, Jena, Germany). The light is again split into the two orthogonal polarized beams by a third GlanLaser polarizing beamsplitter and the back focal plane of the condenser of each beam is focused by a lens onto a quadrant photodiode (QPD). Before each photodiode, the laser beam passes a thin-film polarizer to clean up the polarization signal and reduce crosstalk [35] and a laser line filter which only allows passing of the $1064 \mathrm{~nm}$ trapping light. The currents of the QPDs are converted into Volts showing intensity distributions of the $\mathrm{x}$ - and $\mathrm{y}$ direction of the back focal plane of the optical trap.

For sample illumination, light of a green LED is coupled into the microscope with a dichroic mirror above the condenser. It is passing the second dichroic mirror, which couples the trapping laser into the microscope path and is imaged onto a CCD camera (Coolsnap EZ, Photometrics, Tucson, AZ, USA).

\section{Force-feedback mode 2.2}

So far, the optical trap as described is used to trap an object at a certain position, measure the displacement of the object from the center position of the trapping laser and calculate the according force that is needed to lead to this displacement. To be able to apply a constant force to an object with the trapped particle, one needs to implement a feedback mechanism that keeps the force constant and displaces the particle accordingly (i.e. moving the center position of the trapping laser). Such a force feedback is needed to apply a constant external force to a cell suspended between two optically trapped beads. A force feedback mechanism that allows the repositioning of one of the traps using an acousto-optical deflector (AOD) according to the force signal detected by the QPD of the other trap. The feedback was added to the already existing LabVIEW software control of the trapping setup, "trap commander (TC)" written by Christoph Pieper [36]. The basis of this feedback is the proportional-integral-derivative (PID) algorithm that comes as an extension packet for LabVIEW software. 
In PID control, a process variable and a setpoint are specified. The process variable is the system parameter that should be controlled which is in our case the displacement of the trapped particle from the trap center. The setpoint is the desired value for the parameter that we want to control, here it is the desired displacement/force. The PID controller determines a controller output value, or error signal, and applies it to the controlled system. The system then drives the process variable towards the setpoint value. Figure 2.5 shows a schematic of a PID control process.

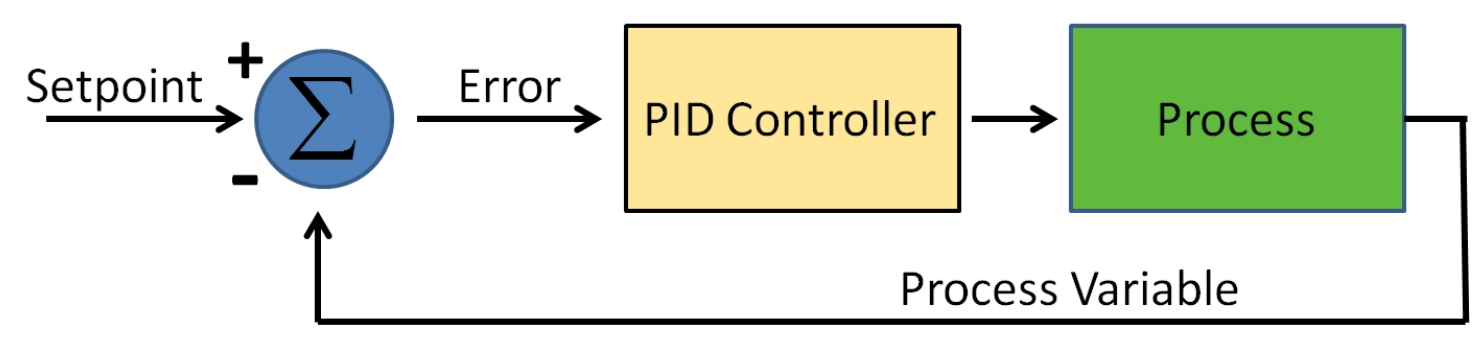

Figure 2.5: Block diagram of a PID controller in a feedback loop.

The PID controller compares the setpoint (SP) to the process variable (PV) to obtain the error $(e)$

$$
e=S P-P V
$$

Then the PID controller calculates the individual action terms for proportional, $u_{P}$, integral, $u_{I}$, and derivative, $u_{D}$ gain.

$$
\begin{aligned}
u_{P}(t) & =K_{c} e \\
u_{I}(t) & =\frac{K_{c}}{T_{i}} \int_{0}^{t} e d t \\
u_{D}(t) & =K_{c} T_{d} \frac{d e}{d t}
\end{aligned}
$$

which combine to the controller action $u(t)$

$$
u(t)=K_{c}\left(e+\frac{1}{T_{i}} \int_{0}^{t} e d t+T_{d} \frac{d e}{d t}\right)
$$

with the controller gain $K_{c}$ the integral time $T_{i}$, also called the rest time, and the derivative time or rate time $T_{d}$. Detailed information about feedback control and PID can be found in [37]. Details about the tuning of the gain- and timing constants of the PID and its implementation in LabVIEV can be found in the "PID Control Toolset User Manual" (National Instruments).

Figure 2.6 shows the block diagram of the force feedback. In the program, the raw data is extracted from the general data stream that travels through the whole trap commander program (light blue cable). From the raw data, the x-ydisplacement signals from the quadrant photodiodes are extracted and split into individual datasets (red box No. 1). A first switch, the feedback should control the x-signal from the direct or the indirect diode. A second switch allows then, to use a low-pass filtering so that the feedback only corrects the signal below the filter 


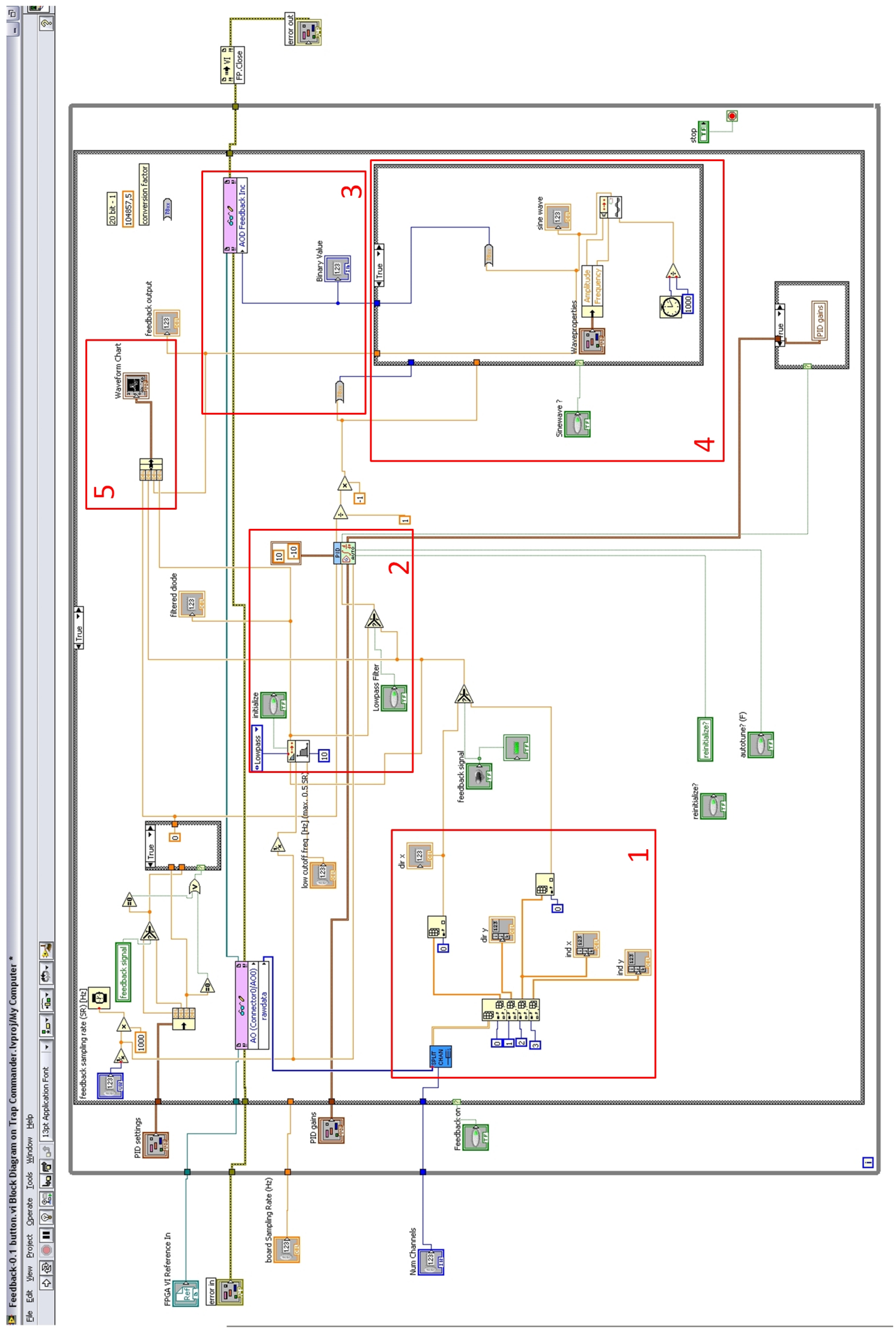

Figure 2.6: Block diagram of the feedback programm. The QPD signal is extracted from the datastream and low-pass filtered. The filtered x-signal of the diode is send as the process variable to a PID controller. The algorithm calculates the error signal which is then fed to the AOD. 
frequency. In general, for the cell experiments, the low-pass filter is set to $30 \mathrm{~Hz}$ since the feedback should only control the low frequency regime where motor proteins are active $(<10 \mathrm{~Hz})$. The low-pass filtered signal is the fed into the PID algorithm as the process variable (red box No. 2). The PID.vi is also fed with the parameters for the PID variables and with the setpoint. The output value of the PID algorithm is then binarized and sent to a variable which is connected with the general data stream and the FPGA board (red box No. 3). The output variable is then connected on the FPGA board with the analog output AO1 which is connected to drive the AOD. There is also the option to connect a sinusoidal signal to the output instead of the feedback signal (red box No. 4). This is useful to calibrate the output signal (Volts) of the FPGA analog output into a displacement signal $(\mu m)$ of the trapping laser by the AOD. Setpoint, diode signal, low-pass filtered diode signal and feedback output (or function generator output) are displayed as a graphical control (red box No. 5). The feedback is designed as a software controlled PID feedback. Therefore it only works, if the trap commander is receiving data from the FPGA board (during preview or measurement). This allows the feedback to work only at lower (software) sampling rates $(<1 \mathrm{kHz})$ which is sufficient enough for the cell experiments. If a faster feedback is needed, the PID control has to be shifted to the FPGA hardware, which is able to work at frequencies of roughly $200 \mathrm{kHz}$. There, the sampling rate is limited probably by the speed of the hardware filters and the speed of sound in the AOD crystal to roughly $60-100 \mathrm{kHz}$. Examples of a FPGA-based PID control for optical traps can be found in [38, 39].

In figure 2.7, the front panel of the feedback is shown. The main part of the window is used by the graphic control of the setpoint, diode signal and PID output. On the left side next to the graph are the controls for the calibration factor of the optical trap $(p N / V)$ and the force setpoint. Below that are the control parameters for the PID gains. On the lower left side of the panel are the control parameters and the switch for the sine-wave generator. Above the graphics panel are the main switch for the feedback, the switch for the low-pass filter and the settings for the filer frequency and sampling rate. Above that is the switch to decide if the feedback should control the $\mathrm{x}$-value of the direct- or indirect trap. On the top right side of the panel are the numerical controls for the graphic display and for the output value of the feedback. Right next to the graphic panel are buttons for autotuning and initializing the feedback.

\subsection{Measuring cellular force fluctuations}

To measure force fluctuations and elasticity of the cells, methods of active and passive microrheology are used. The dual optical trap is first used to capture two fibronectin-coated beads and attach them to opposite sides of a rounded-up cell (fig. 2.8). Each bead was allowed to bind to the cell for approximately 5 minutes and form a tight adhesion domain. The two beads now mimic an extracellular matrix for the cell and allow it to transmit forces to the beads that result in position changes of the beads. These position fluctuations were recorded by the quadrant photodiodes 


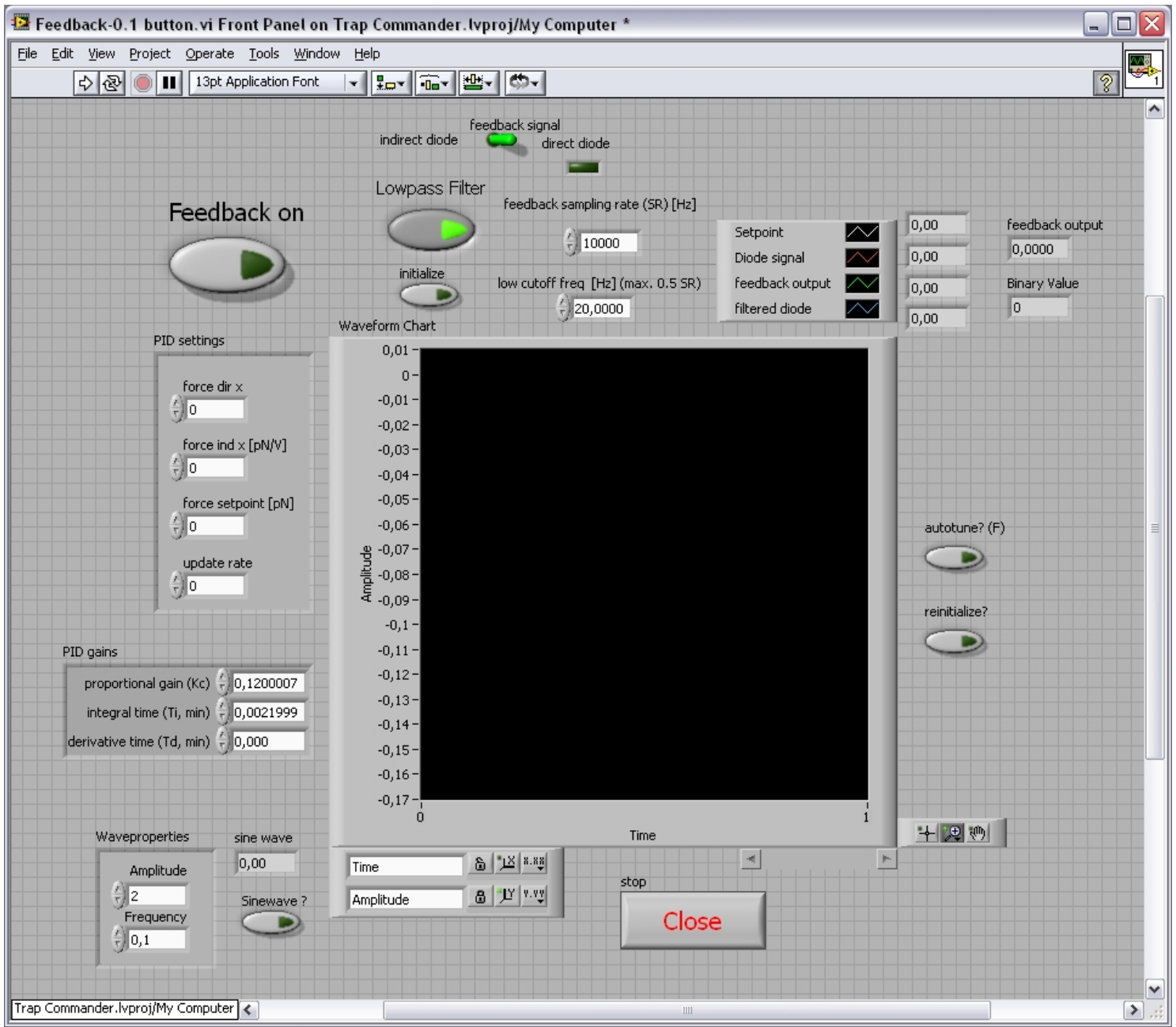

Figure 2.7: Front panel of the feedback program. Graphical control screen of the setpoint, diode signal and feedback output in the middle. Control panels for PID settings, low-pass filter settings and general program start-stop buttons on the side.

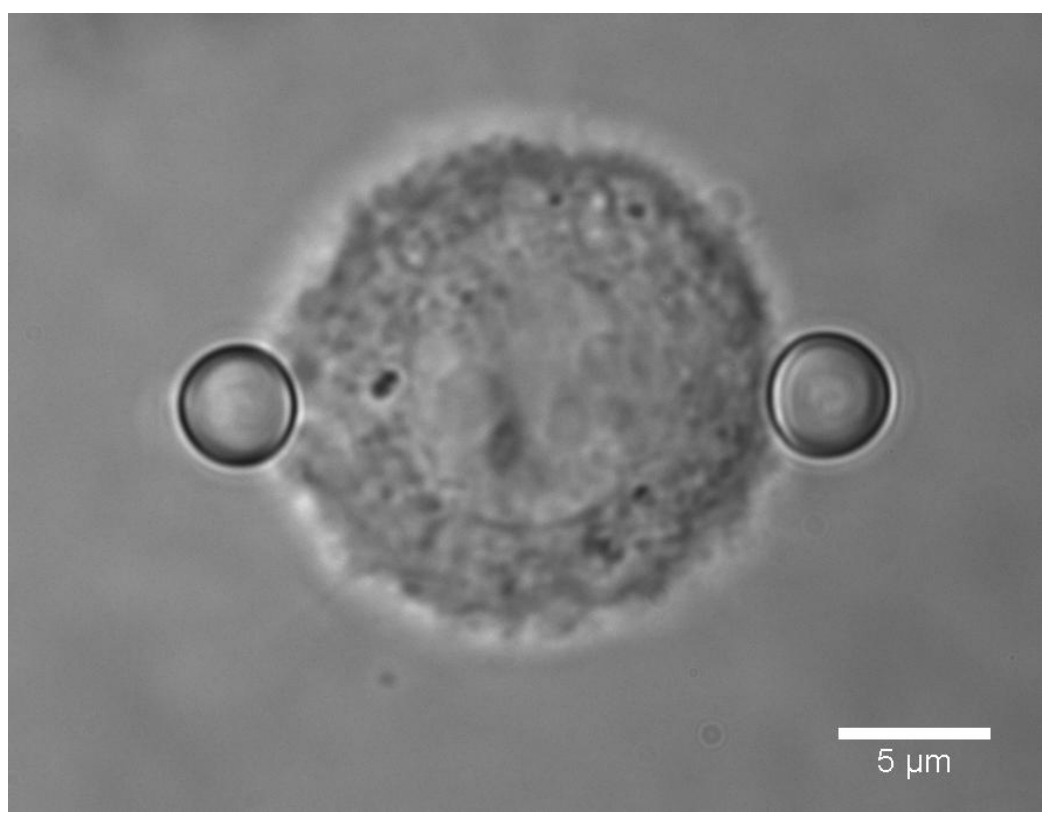

Figure 2.8: Fibroblast suspended between two optically trapped fibronectin-coated polystyrene beads. 


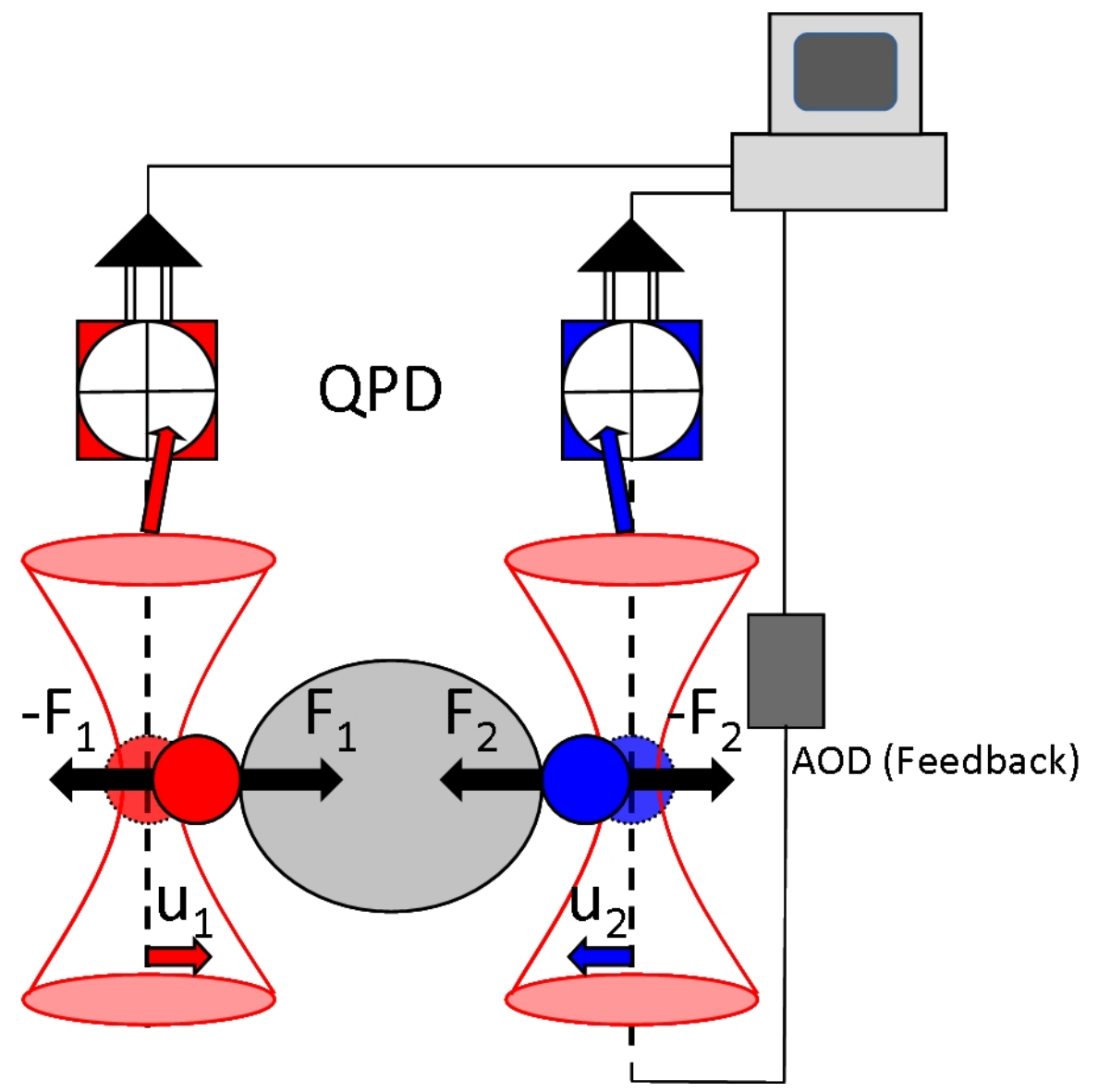

Figure 2.9: Schematic of the setup: a cell (grey) exerts forces $F_{1}$ and $F_{2}$ on the two attached beads (red and blue). The corresponding displacements of the beads $\left(u_{1}\right.$ and $\left.u_{2}\right)$ in the traps are detected by QPDs and recorded via FPGA I/O board and LabVIEW software. In feedback mode, the computer controls the AOD and displaces one of the traps (blue) to keep the force measured on the red bead constant.

placed in the conjugate plane to the back-focal plane of the condenser lens of the microscope [30]. Voltage signals from the QPDs were acquired and digitized using a field programmable gate array (FPGA) and LabView Software (NI PXI-7833R, National Instruments) (figure 2.9). This measurement configuration using the dual optical trap approach has also the advantage that one can easily change the external stiffness that the cells feel by adjusting the trapping (laser) power. To distinguish the non-equilibrium cellular force fluctuations from Brownian motion background, we have to combine active and passive microrheology. In the passive mode, keeping the beads at constant positions and following their displacements, one obtains the full fluctuation spectrum, including the one due to thermal forces. In the active mode, one oscillates one of the beads at a single frequency and detects the resulting fluctuations of the other one. A lock-in amplifier then calculates directly a material response of the cell at that frequency. The fluctuation-dissipation theorem allows us to calculate the apparent thermal fluctuation spectrum from the response curve. A more detailed description of microrheology and its application for cells can be found in [40][41]. 


\section{Passive method}

For passive measurements, we recorded the position fluctuations of the two beads $u_{i}(t)$ and calculated the Fourier transform of the cross correlation of the bead positions, the powerspectral density (PSD)

$$
P S D(\omega)=\left\langle u_{1}(\omega) u_{2}^{*}(\omega)\right\rangle_{t o t}:=\int_{-\infty}^{\infty} d t^{\prime}\left(u_{1}\left(t+t^{\prime}\right) u_{2}\left(t^{\prime}\right)\right) \exp (i \omega t)
$$

with custom written MATLAB (MathWorks, Natick, MA, USA) programs from the "'MR toolbox"' previously described in [42]. The position cross-correlation powerspectrum is then calibrated with the trap stiffness $k$ into a force spectrum. To get an estimate of the total force that the cell is generating, the force spectrum is integrated in the range of $0.1-10 \mathrm{~Hz}$ to get $\sum_{0.1}^{10} F F^{*}$.

\section{Active method}

To measure the response function of the cell, one of the traps is oscillated with a driving force $f_{2}$ by the AOD. The driving signal and the corresponding QPD signal of the stationary trap $u_{1}$ are fed into a lock-in amplifier, which calculates the complex response $A_{1,2}^{*}$ of the system

$$
A_{1,2}^{*}=A_{1,2}^{\prime}+i A_{1,2}^{\prime \prime}=\frac{u_{1}}{f_{2}}
$$

We can use the fluctuation-dissipation theorem (FDT) to compare the fluctuations of our system with the imaginary part of the response function

$$
\left\langle u_{1}(\omega) u_{2}^{*}(\omega)\right\rangle=\frac{2 k_{B} T}{\omega} A_{1,2}^{\prime \prime}
$$

In equilibrium, both sides of the equation are equal. However, in our system, the cells are generating force fluctuations consuming energy, which makes our system highly non-equilibrium. Still, we can use the FDT to get an estimate of the thermal fluctuations that we calculate from the response function

$$
\left\langle u_{1} u_{2}^{*}\right\rangle_{\text {thermal }}=\frac{2 k_{B} T}{\omega} A_{1,2}^{\prime \prime}
$$

and subtract that thermal spectrum from the total fluctuation spectrum, that we obtain from the passive method, leaving only the non-equilibrium part of the fluctuation spectrum

$$
\left\langle u_{1} u_{2}^{*}\right\rangle_{\text {non-equi }}=\left\langle u_{1} u_{2}^{*}\right\rangle_{\text {tot }}-\left\langle u_{1} u_{2}^{*}\right\rangle_{\text {thermal }}
$$

If we compare the response function, calculated from the fluctuations using the FDT (passive method) and the real response function (active method) we see, the real part of the response function roughly overlaps. However, we see a large deviation in the imaginary part starting roughly below $10 \mathrm{~Hz}$. This shows that the non-equilibrium fluctuations generated by the cell are only dominant below $10 \mathrm{~Hz}$. Whereas the 


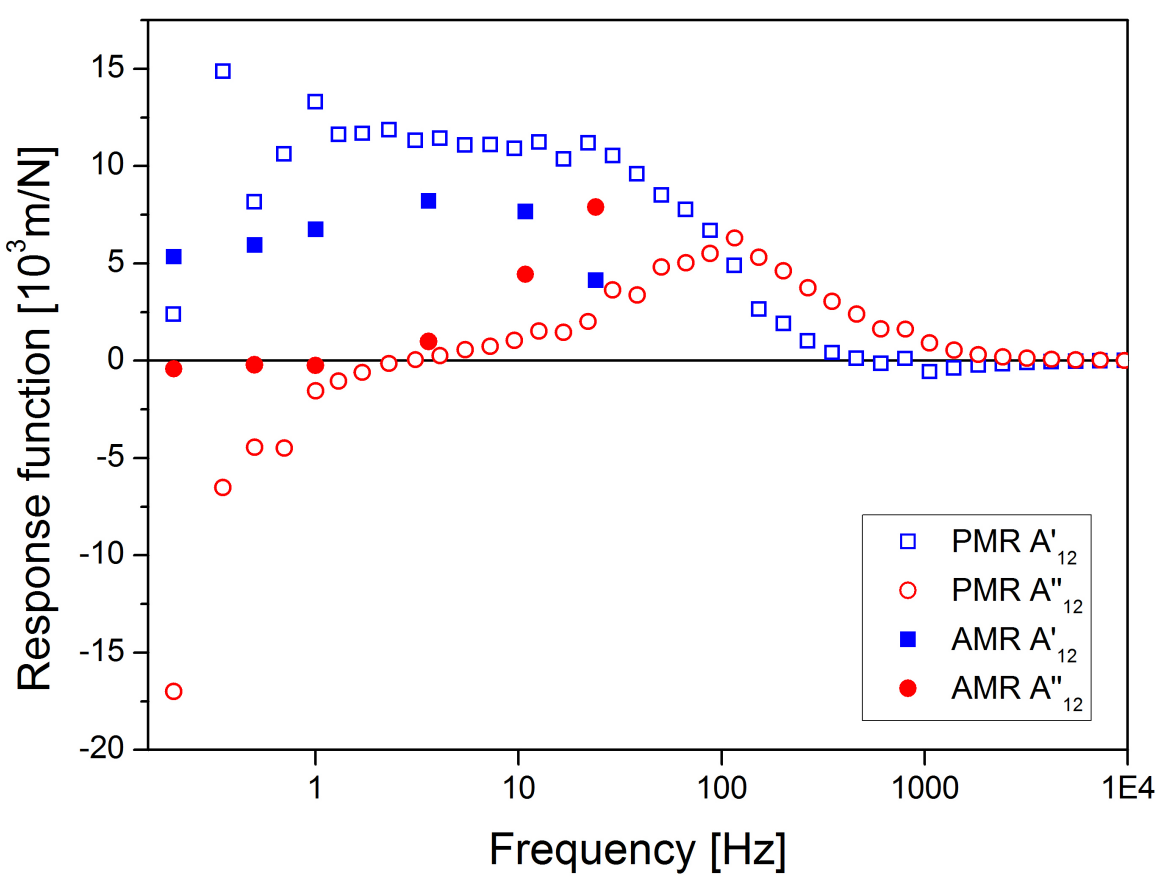

Figure 2.10: Response function of a 3T3 fibroblast. Open symbols show the fluctuation measurements transferred into a response using the FDT (PMR, passive method). The close symbols show the response of the cell (AMR, active method). Blue squares for the real part of the response functions and red circles for the imaginary part of the response function.

imaginary part of the fluctuations show a large negative response, the real response is roughly zero (figure 2.10). This shows that the thermal fluctuations in this frequency regime are negligible and that the total force spectrum is dominated by the nonequilibrium fluctuations of the cell, therefore it is safe to assume

$$
\left\langle u_{1} u_{2}^{*}\right\rangle_{\text {non-equi }} \approx\left\langle u_{1} u_{2}^{*}\right\rangle_{\text {tot }} \text { for } \quad f \leq 10 H z
$$

\section{$2.4 \quad$ Elasticity measurements}

For the active compliance measurements, the AOD was used to oscillate spatially one of the optical traps, while keeping the other one at constant position. Displacements of approximately $500 \mathrm{~nm}$ amplitude with a frequency of $0.2 \mathrm{~Hz}$ were applied, which remains in the linear response regime of the cell [40]. The position of both beads within the optical traps $\left(u_{i}(t)\right)$ and the center position of the oscillating trap $(d)$ are recorded and the applied force $F=k\left(u_{2}-u_{1}\right)$ is calculated (figure 2.11). The driving amplitude of the AOD-steered trap minus the difference between displacements $u_{i}$ gives the effective elongation of the cell $\Delta l=d-\left(u_{1}-u_{2}\right)$. By approximating the cell as a linear-elastic element, we can use Hooke's law

$$
F=k_{\text {cell }} \Delta l
$$




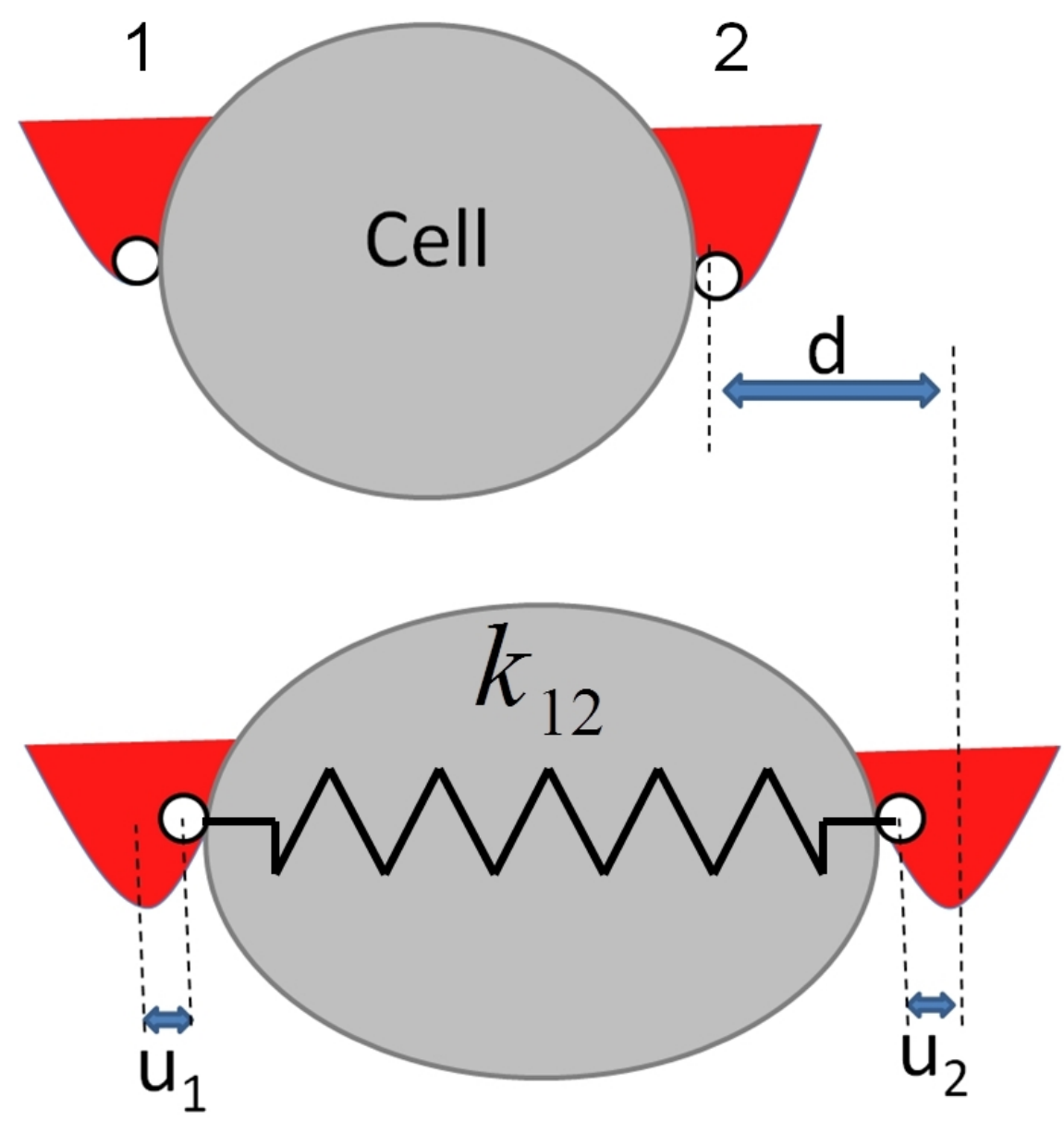

Figure 2.11: Schematic of an elasticity measurement. Upon a stretching event, the moving trap is displaced by the AOD by a distance $d$. Both beads are displaced from the trap center by a distance $u_{1}$ and $u_{2}$, respectively. The cell is modeled as an elastic spring with spring constant $k_{12}$.

and obtain an elastic spring constant $k_{\text {cell }}$ from force-elongation plots for each individual cell.

\section{Cell culture}

NIH 3T3 cells (ACC 173, DSMZ, Braunschweig, Germany) were cultured in Dulbecco's modified Eagle's Medium (DMEM, D6046, Sigma-Aldrich, St.Louis, MO, USA) with $10 \%$ fetal bovine serum (FBS, F0244, Sigma) and 1\% penicillin-streptomycin (17-602E, Lonza, Basel, Switzerland) in $75 \mathrm{~cm}^{2}$ culture flasks (83.1813, Saarstedt $\mathrm{AG}$, Nümbrecht, Germany) at $37^{\circ} \mathrm{C}$ and $5 \% \mathrm{CO}_{2}$. Cells were grown up to a confluency of roughly $80 \%$ and passaged in new culture flasks using $0.05 \%$ trypsin $(59417 \mathrm{C}$, Sigma) every 2-3 days with a density of 125,000 cells per flask.

For experiments, cells grown to roughly $80 \%$ confluence were trypsinized for 3 min at $37^{\circ} \mathrm{C}$ and centrifuged after adding DMEM with FBS to inhibit the trypsin. 
The pellet was resuspended in $\mathrm{CO}_{2}$ independent medium (18045-054, Life Technologies, Darmstadt, Germany) also supplemented with 10\% FBS and stored on ice until use within $8 \mathrm{~h}$.

For the drug perturbation experiments, trypsinized cells were incubated in a test tube with either $100 \mu \mathrm{M}$ blebbistatin (racemic mixture, 203389, Merck, Darmstadt, Germany) or $3 \mu \mathrm{M}$ nocodazole (M104, Sigma) for $30 \mathrm{~min}$ at room temperature prior to the experiment.

\section{Transfection}

For transfection with the 3D nucleofector (Lonza), cells grown to $80 \%$ confluence were trypsinized as previously described and centrifuged. The cells were counted and 1 Mio cells were resuspended in the desired transfection buffer (SE-buffer for NIH 3T3, P2-buffer for cardiac fibroblasts) (Lonza). DNA of the desired fluorescent fusion protein, LifeAct-RFP (ibidi, Martinsried, Germany) [43] and/or non-muscleMyosin-IIa (gift from R.Adelstein, Addgene plasmid \# 11347 [44]) was added to a total amount of $3 \mu \mathrm{g}$ in the sample. The cell solution was transferred to transfection vessels and transfected in the 3D nucleofector with program CA137. The solution was incubated for 5 minutes after transfection, then $100 \mu \mathrm{l}$ of pre-warmed medium was added and the solution was transferred in a culture flask.

\section{Primary fibroblasts}

Cardiac fibroblasts (CFB), human foreskin fibroblasts (HFF), gingival fibroblasts (GFF) and human induced pluripotent stem cell derived fibroblasts were cultured in the lab of Prof. Zimmermann at the department of pharmacology in the university medical center in Göttingen. Cells were transported to our lab in petri dishes with culture medium in an isolation box. They were detached using the same trypsin protocol that was used for the NIH 3T3 fibroblast cell line. All experiments were done in $10 \%$ FBS supplemented $\mathrm{CO}_{2}$ independent DMEM at room temperature. Non-muscle myosin II perturbations were done adding $100 \mu \mathrm{M}$ blebbistatin to the sample as described before.

\section{$2.6 \quad$ Vesicle preparation}

Giant unilamellar vesicles (GUVs) were prepared from a mixture containing $95 \%$ of 1,2-dioleoyl-sn-glycero-3-phosphocholine (DOPC) and 5\% 1,2-dioleoyl-sn-glycero-3phosphoethanolamine-N-(cap biotinyl) (DOPE-B-Cap) (850375, 870273, both Avanti Polar Lipids, Alabaster, AL, USA) by electroswelling in a solution of $100 \mathrm{mM}$ glucose (X997.2, Carl Roth, Karlsruhe, Germany) and $1 \mathrm{mM} \mathrm{NaCl}$ (1064041000, Merck) as described in [45] and stored in a solution of $100 \mathrm{mM}$ succrose (9286.1, Roth) and 1 $\mathrm{mM} \mathrm{NaCl}$ at $4^{\circ} \mathrm{C}$ for up to one week. 


\section{Force fluctuations of suspended cells}

\section{\begin{tabular}{l|l} 
Introduction & 3.1
\end{tabular}}

Mechanical interactions and forces that cells exert and feel govern many of their processes such as substrate- or cell-cell adhesion, locomotion and cell division [46, 11 , 47, 48, 16, 8]. Cells constantly communicate with each other through forces. They generate forces to move through tissue but also to probe the mechanical response of their environment. A focus in research of cell forces has been performed on the influence of substrate stiffness to shape and internal structure of cells $[49,50$, 51]. The most prominent structures that develop in the acto-myosin cell cortex are contractile stress fibers that can be highly ordered [51].

Cells generate contractile force mostly through their acto-myosin network where myosin motors create contractile stress across actin bundles by hydrolyzing ATP [52]. Another source of force generation is polymerization and depolymerization of the cytoskeletal filaments microtubules and actin [53]. Viscoelastic properties along with microscopic force generation determine the dynamics of such 'active matter'. Reconstituted in vitro model systems including networks encapsulated in a lipid envelope mimicking cellular geometry [54] have been successfully used to study the mechanical properties of such systems $[55,56]$. However, it is still challenging to measure forces and mechanical properties of living cells accurately. An easy way to measure these properties has been to use traction force microscopy on substrateadherent cultured cells [57], a rather non-physiological situation for most cell types. A tool to probe suspended cells is the optical stretcher $[58,59]$ but it has the drawback that cells are not attached to any substrate, not being able to form focal adhesions. It is also not possible to measure cell forces.

In this study, we further develop an approach that we have introduced recently, using active and passive microrheology to probe force generation and elastic response of the cell simultaneously [40, 41]. We attach micrometer-sized polystyrene beads to a suspended cell with a dual optical trap (figure 1a). The position of the beads is tracked with high bandwidth using laser interferometry (see figure 2.9). This approach allows us to probe the mechanical properties of suspended cells with oscillatory forces and in the same experiment measure the force fluctuations that the cell generates. This approach allows us to measure, in contrast to experiments that rely on surface adherent cells, the total force transmitted to the outside world. 


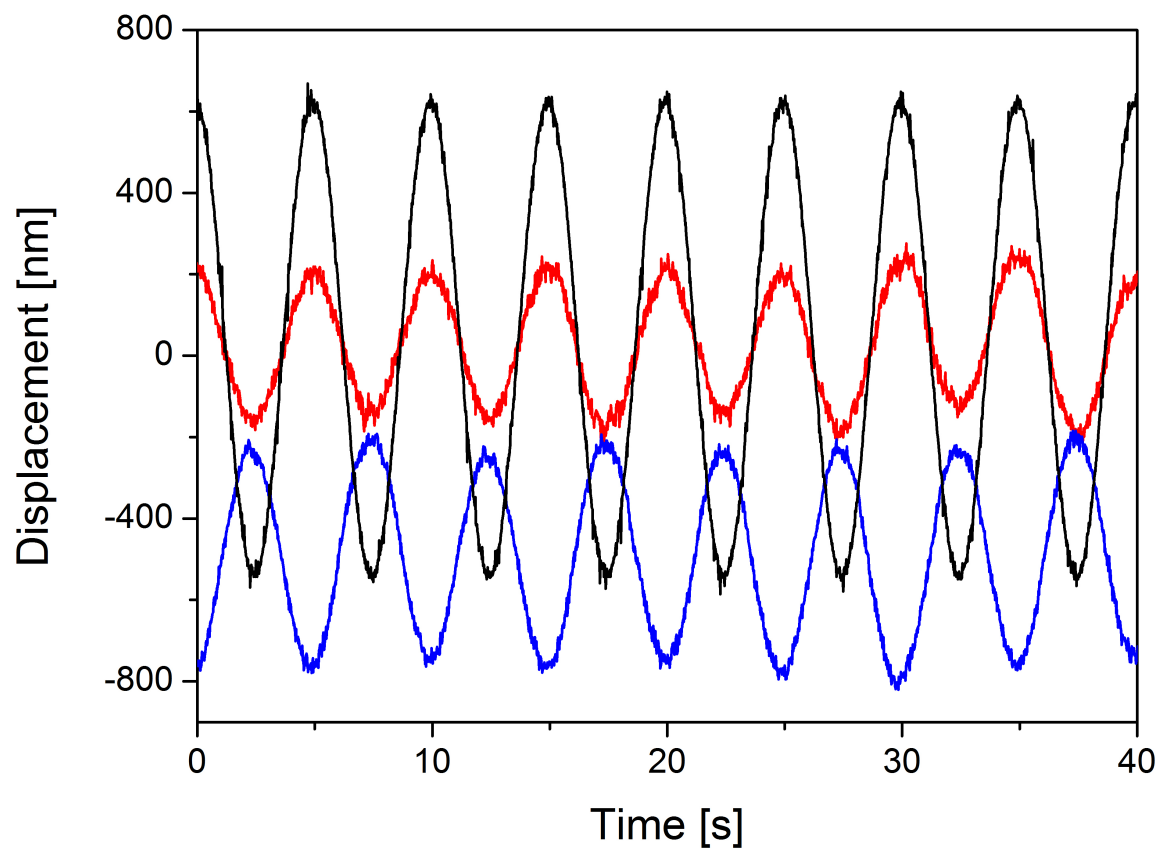

Figure 3.1: Example of the time courses of displacements of the two particles from the trap center of the stationary (red) and oscillating (blue) trap and the driving amplitude of the AOD steered trap (black). Driving frequency $0.2 \mathrm{~Hz}$.

In rounded cells, actin and myosin are mainly confined to the less than $1 \mathrm{~mm}$ thick cellular cortex $[60,61,62]$. Due to the surface tension that is in competition with osmotic pressure, cells maintain a round shape [16, 63, 64]. Cells have the possibility to store excess lipid membrane area in different types of surface invaginations $[65,66]$ unlike lipid vesicles that are used as model systems. It has been shown that myosin motors are able to contract and compact the actin networks [67, 68], therefore surface tension is generated by the acto-myosin network. Here, we demonstrate that the cortical acto-myosin network is the key cytoskeletal component generating contractile force fluctuations and providing mechanical strength, whereas the contribution of the microtubule network is negligible. Most results in this chapter have been published in [69].

\subsection{Elasticity}

First, we use the active mode to measure effective cell stiffness (section 2.4). We apply oscillatory forces using the acousto optic deflector to move one of the optical traps (figure 2.9 and 2.11). Displacements of approximately 500-600 nm with a frequency of $0.2 \mathrm{~Hz}$ were applied, which remains within the linear-elastic response regime of the cell [40]. Positions of both beads and the center of the oscillating trap were recorded and the force applied to the cell and its elongation was calculated. In figure 3.1 an example of the displacement signals $u_{1}(t)$ and $u_{2}(t)$ of the two particles from the trap center of the stationary and oscillating trap, and the driving amplitude 
of the AOD-steered trap $d$ are shown. For each measurement, the mean displacement of one oscillation period is calculated. From this plot, we calculate the effective elongation of the cell $\Delta l=d-\left(u_{1}-u_{2}\right)$ and the applied force $F=k\left(u_{2}-u_{1}\right)$. We approximate the cell as a linear-elastic element and use Hooke's law $F=-k_{\text {cell }} \Delta l$ to obtain an effective spring constant $k_{\text {cell }}$ from force-elongation plots of each individual cell. Figure 3.2A shows an example of such a force-elongation relation for a 3T3 fibroblast in normal medium conditions (control). Averaging over 10 individual cells yields an effective spring constant of $k_{\text {cell }}=9.5 \pm 2.5 \times 10^{-5} \mathrm{Nm}^{-1}$
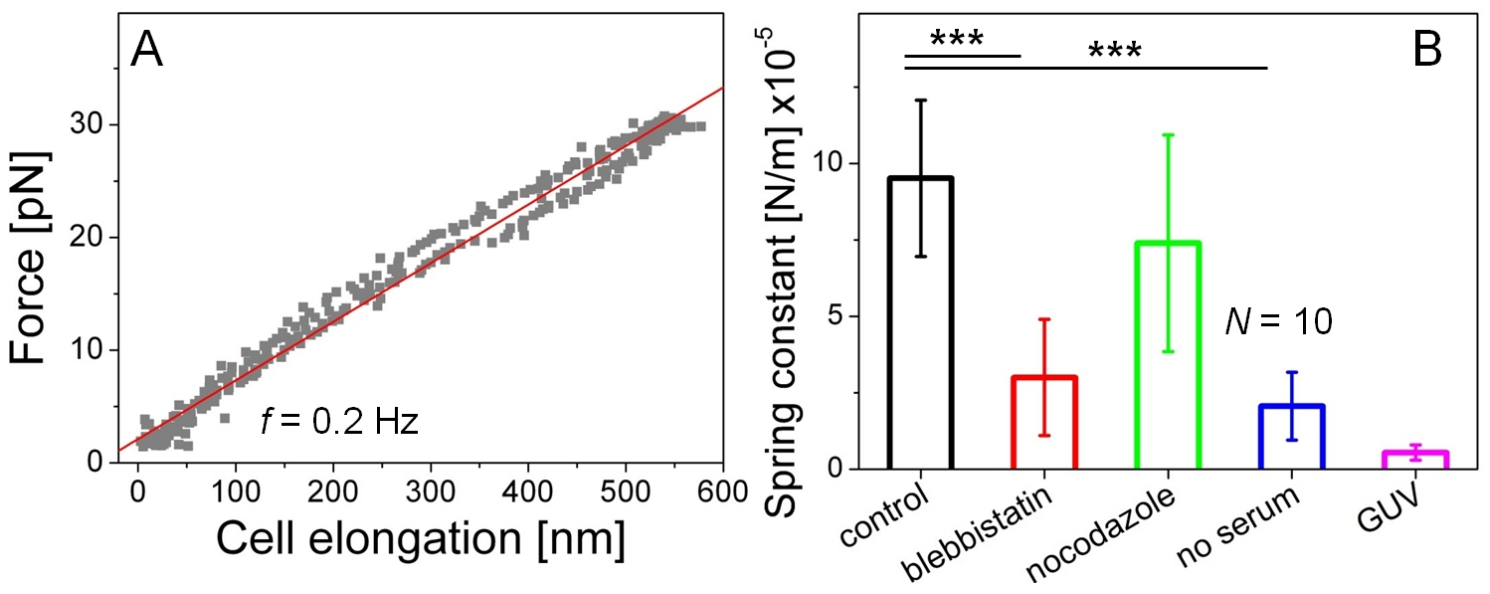

Figure 3.2: Active measurement of cell elasticity. A: Force-elongation plot from a periodic stretching experiment, averaged over five periods and linear fit to get $k_{\text {cell }}$. B: Effective cell spring constants of fibroblast cells under control conditions (black), after treatment with $100 \mu \mathrm{M}$ blebbistatin (red), $3 \mu \mathrm{M}$ nocodazole (green) and in serum-free medium (blue). Effective spring constant of DOPC/DOPE-biotinyl giant lamellar vesicles (GUV) (purple). Ten cells measured for each condition (GUV, $\mathrm{N}=6$ ).

We did active deformation experiments to determine the effective spring constant $k_{\text {cell }}$ using biochemical perturbations. This allows us to distinguish the contributions of actin, myosin and microtubules to cellular compliance (figure 3.2B). We see a significant threefold decrease in cell stiffness $k_{\text {cell }}=3.1 \pm 1.5 \times 10^{-5} \mathrm{Nm}^{-1}$ after blocking non-muscle myosin II motors (NMM II) with $100 \mu \mathrm{M}$ blebbistatin in comparison to cells in control conditions (t-test, significance level 99.9\%). This result clearly confirms a major contribution of the cortical acto-myosin network to cellular rigidity and integrity. Microtubules are stiff rods with a persistence length of $l_{p} \approx 5 \mathrm{~mm}$ [70], therefore their more evenly distributed network might play a role in the overall mechanical response of the cell. We treated the cells with $3 \mu \mathrm{M}$ nocodazole to depolymerize the microtubules but see only a slightly smaller stiffness $k_{\text {cell }}=7.5 \pm 4.1 \times 10^{-5} \mathrm{Nm}^{-1}$ than untreated cells which was not statistically significant. Although subtle changes might be not detectable in our setup with an error margin of roughly $30 \%$, we see that depolymerizing microtubules has no notably effect on cell compliance. It is known that cells on substrates in low-serum or serum-free conditions have significantly reduced contractile forces [71, 72]. We tested the effect of serum-starvation and found that cells show a significant reduction (p>99.9\%) in cell stiffness $k_{\text {cell }}=2.1 \pm 1.2 \times 10^{-5} \mathrm{Nm}^{-1}$ compared to the control population after only 30 minutes. This reduction in stiffness is comparable to the one seen after blebbistatin treatment. This is an indication that serum starvation is an efficient method to shut down cellular force fluctuations. 
We used giant unilamellar vesicles (GUV) as a model system to estimate the possible contribution of the plasma membrane to the elastic response of the cell. We found that the spring constant of GUV was significantly ( $\mathrm{p}>99.9 \%$ ) 30-fold lower compared to the effective spring constant of control cells. In an osmotically stretched GUV, uniaxial deformation would couple to both bending and stretching of the bilayer against osmotic pressure. Cells, however, have access to membrane stores and bilayer stretching is therefore not relevant here. So the results for GUV merely provide an upper limit for the contribution of bilayer bending. Nevertheless it is safe to conclude that the mechanical contribution from the lipid bilayer is negligible due to its large difference compared with the acto-myosin network.

\subsection{Force fluctuations}

It is interesting to compare cell compliance with force fluctuations. Therefore, we used the passive mode to measure force fluctuations of the cells for different biochemical perturbations. We used stationary optical traps and attach the beads to cells at positions that correspond to initially zero force (figure 3.3). For longer timescales or

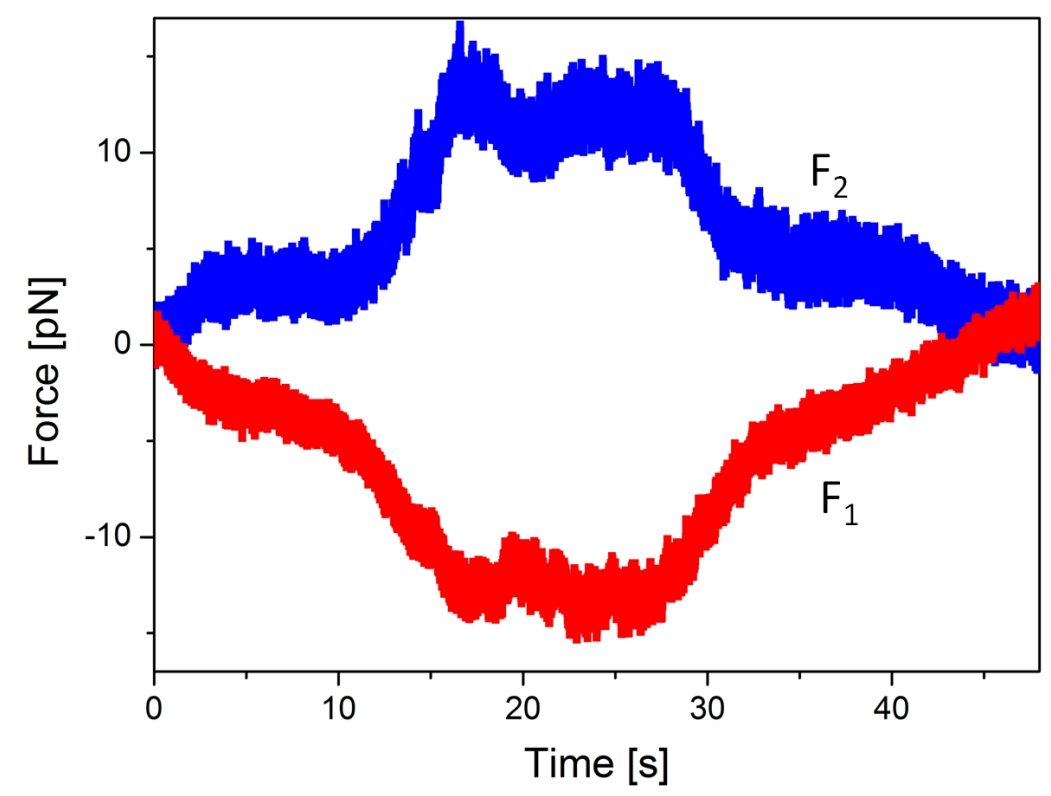

Figure 3.3: Force curves $F_{i}=k \cdot u_{i}$ of two beads attached to opposite sides of a fibroblast cell.

low frequencies, viscous drag is not relevant in the sample, so the force that the cell exerts on the beads must be equal and opposite $F_{1}=-F_{2}$ since the cell has no other anchor point. We calculated the Fourier transform of the of the cross-correlation of the two displacement time traces $\left\langle u_{1}(\omega) u_{2}^{*}(\omega)\right\rangle$ and obtain the corresponding power spectral density (PSD) of the force fluctuations $\left\langle F_{1} F_{2}^{*}\right\rangle=k^{2}\left\langle u_{1} u_{2}^{*}\right\rangle$ by multiplication with the trap stiffnesses who are set to be equal. $\left\langle F_{1} F_{2}^{*}\right\rangle$ is always a proper, positive PSD because the forces have to be opposite and equal in contrast to the amplitudes $u_{1}$ and $u_{2}$ in the case of non-equal trap stiffness. Consistent with earlier results $[56,40]$, we find that the frequency dependence of the PSD follows a scaling law $\left\langle F F^{*}\right\rangle=k^{2}\left\langle u_{1} u_{2}^{*}\right\rangle \propto \omega^{-2}$ (figure 3.4) which corresponds to a mean squared 
displacement scaling linearly in time. Simple thermal diffusion in a purely viscous

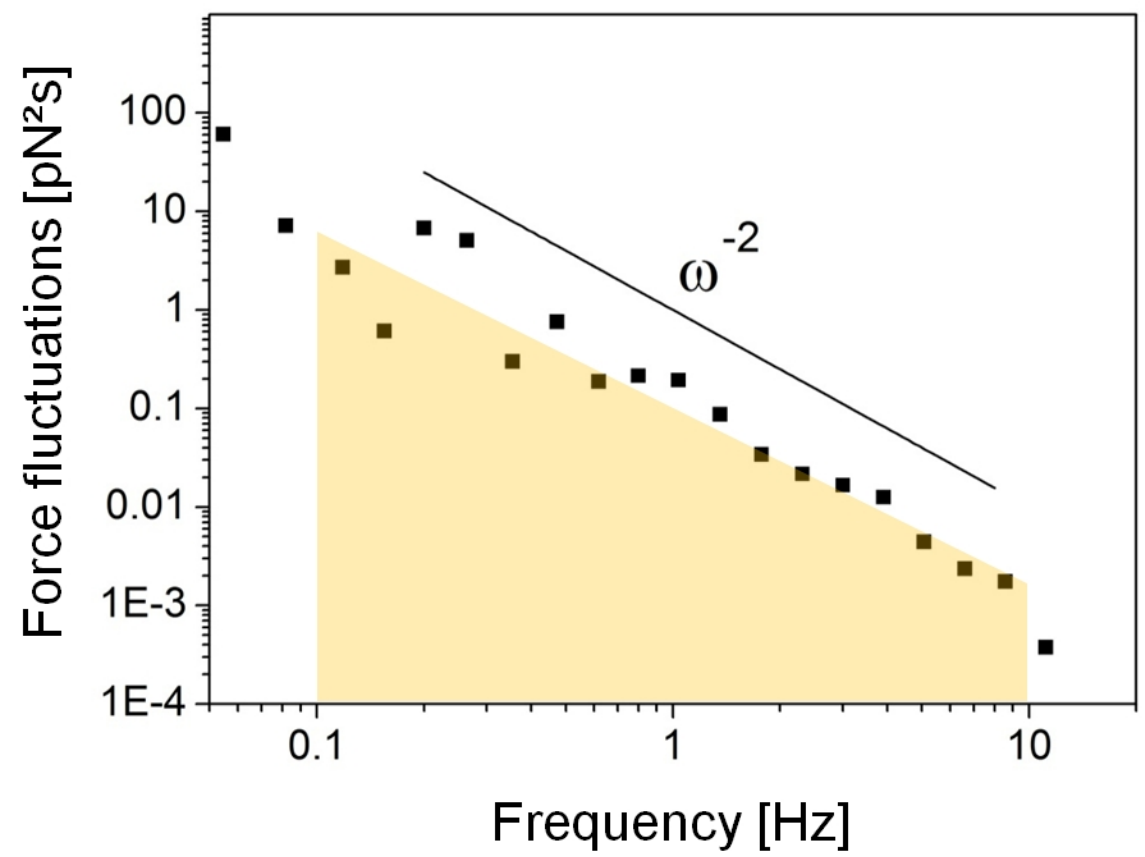

Figure 3.4: Force spectrum of a fibroblasts cell. The orange box marks the integration window for each measurement to get the sum of force fluctuations $k^{2} \sum u_{1}(\omega) u_{2}(\omega)$. The solid black line represents a $\omega^{-2}$ power law.

environment would generate exactly the same scaling law. However, we know from the active compliance measurements that the beads are attached to a predominantly elastic cell. In this environment, the cross-correlation of thermal fluctuations would be almost flat in the frequency domain [73]. Therefore, the observed scaling must be caused by the non-equilibrium stress fluctuations generated by internal motor activity.

Our experiments are done at room temperature $\left(23^{\circ} \mathrm{C}\right)$. Therefore we checked if the cells behave different at $37^{\circ} \mathrm{C}$. Although we see more active and even dividing cells, we do not see any statistically significant difference in force generation between $23^{\circ} \mathrm{C}$ and $37^{\circ} \mathrm{C}$ (figure 3.5).

We integrate the force PSDs in the range from 0.1 to $10 \mathrm{~Hz}$, where we see nonequilibrium fluctuations, to get a quantitative estimate of the total cellular force. Most parts of the motor driven non-equilibrium are covered with the integration range. We also checked if the force fluctuations of fibroblast cells follow the same spring model that was introduced in earlier work [40]. We plotted the total cellular force versus trap stiffness (figure 3.6) and found that the force increases, as expected from the model with trap stiffness and could therefore confirm the previous work. Briefly, the model explains that more cell-generated force is transmitted to the optical traps if the trap stiffness is higher. At a threshold stiffness of roughly $2 \times 10^{-5}$ $\mathrm{N} / \mathrm{m}$, the total transmitted force reached a plateau which means that it approached the effective cellular stiffness, consistent with the results of the active measurement.

We use drug perturbation experiments to test the contribution of different key components to the total force production of cells. Therefore, we plotted experiments 


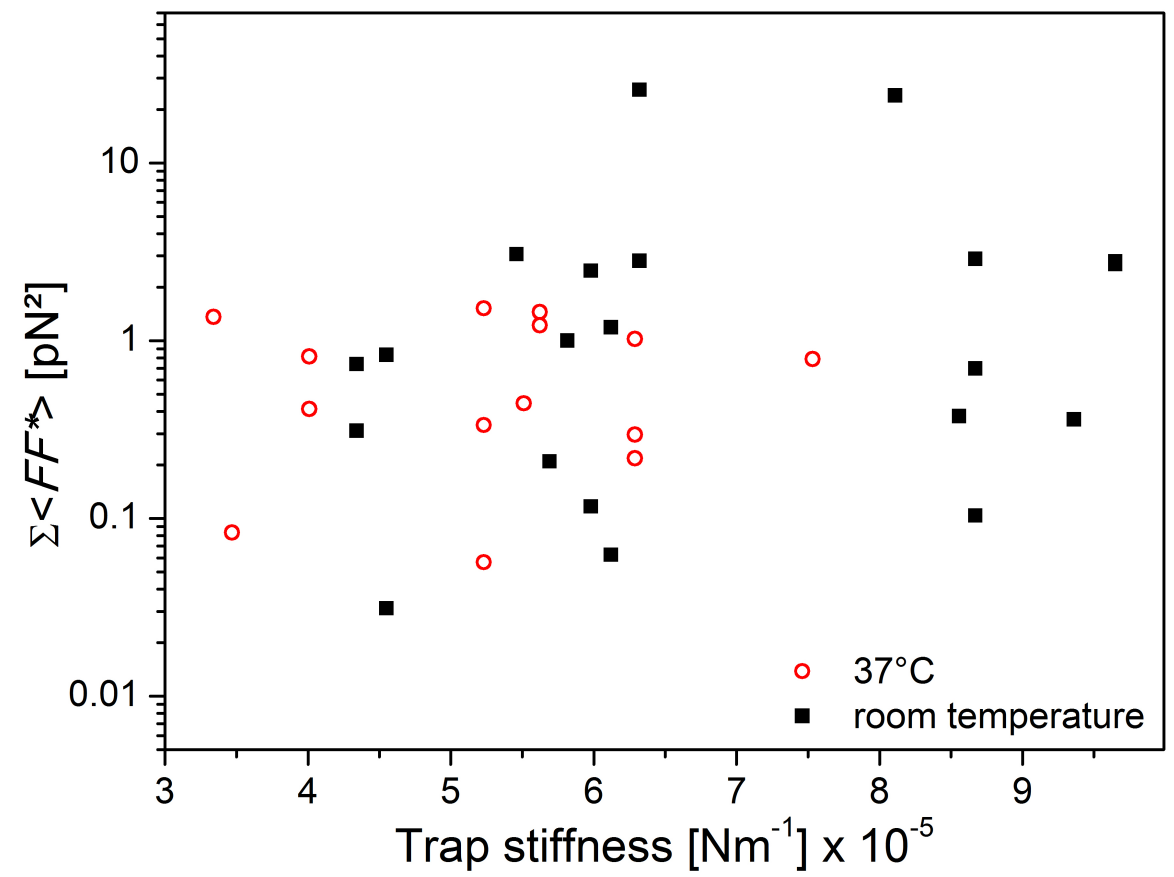

Figure 3.5: Force fluctuations of fibroblast cells at different trap stiffness. Force fluctuations at room temperature (black) and at $37^{\circ} \mathrm{C}$ (red).
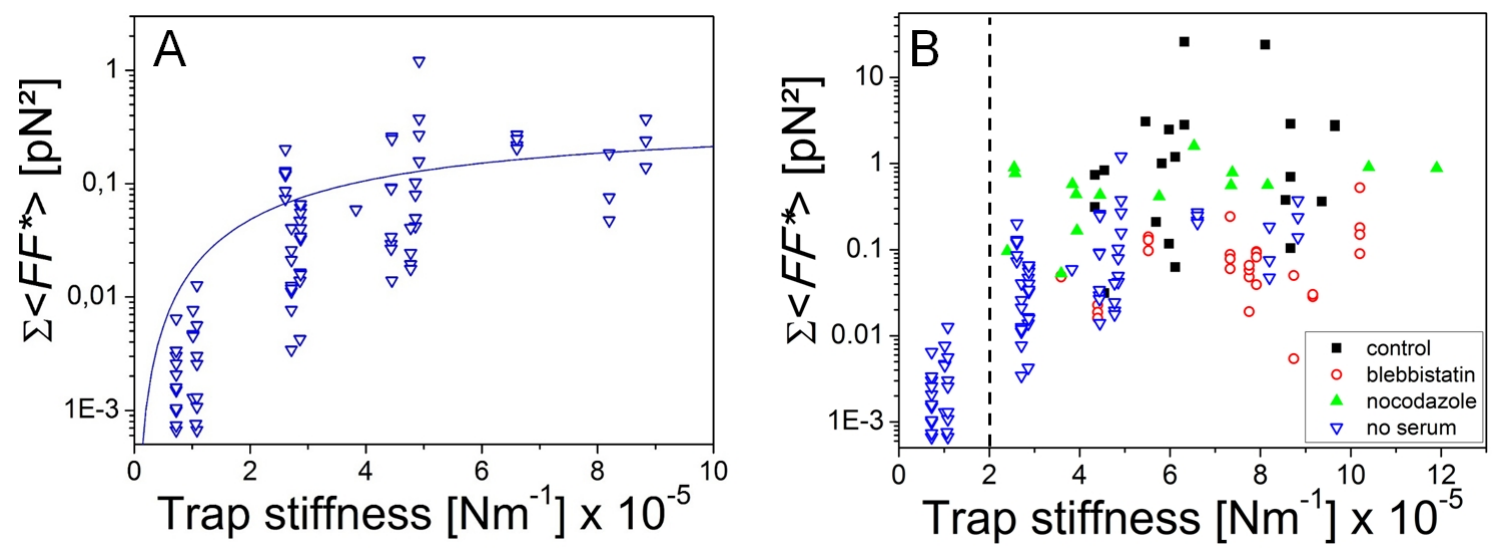

Figure 3.6: Force fluctuations of fibroblast cells plotted versus trap stiffness. A) Fibroblasts in serum-free medium. Each point shows the total force fluctuations of an individual cell obtained by integration. The fit shows the spring model from [40]. B) Total force fluctuations plotted versus trap stiffness of fibroblasts treated with different biochemical perturbations. For averaging in a force histogram, only data points from the plateau region above a trap stiffness of $2 \times 10^{-5}$ $\mathrm{N} / \mathrm{m}$ are used. 
at trap stiffnesses higher than $2 \times 10^{-5} \mathrm{~N} / \mathrm{m}$ in normalized histograms of integrated cellular force fluctuations and calculated the mean values of integrated force fluctuations (figure 3.7). For untreated 3T3 fibroblasts (control), we find a value of
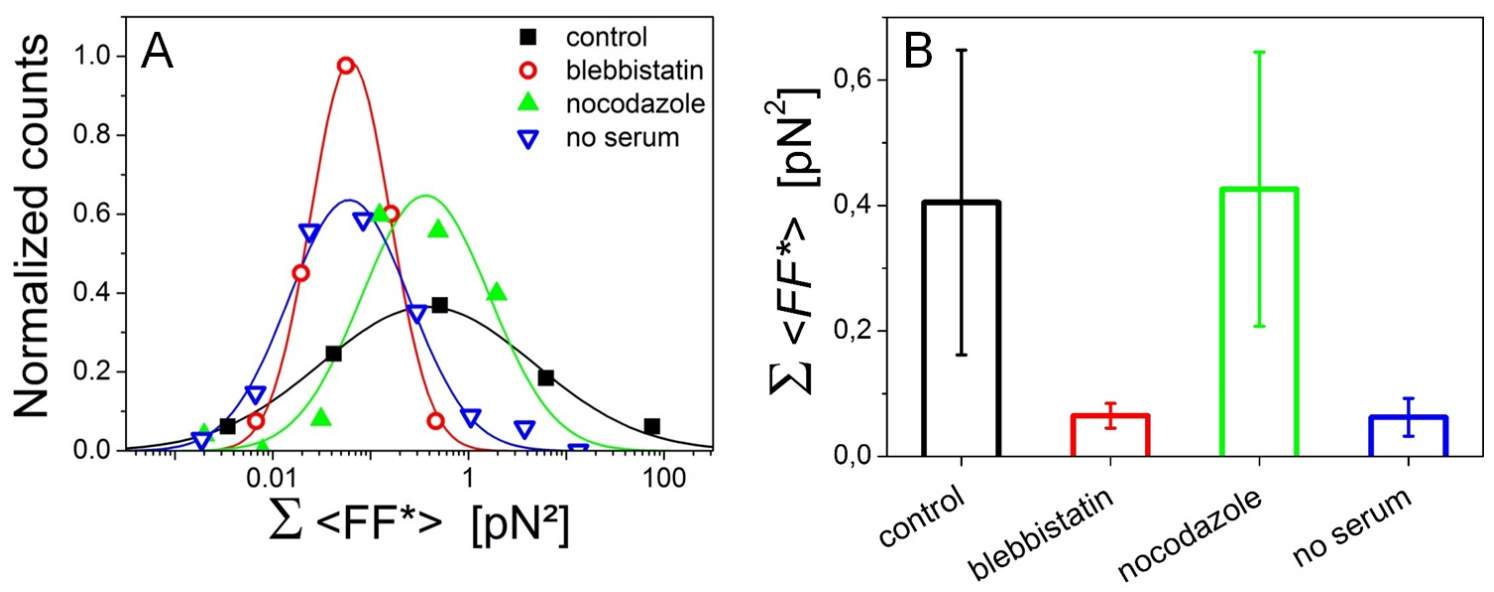

Figure 3.7: Cellular force fluctuations of fibroblasts at different biochemical perturbations. A) Histograms of integrated force fluctuations $(0.1-10 \mathrm{~Hz})$ of control cells (black) and of cells treated with $100 \mu \mathrm{M}$ blebbistatin (red), $3 \mu \mathrm{M}$ nocodazole (green) and in serum free medium (blue). B) Integrated force fluctuations, averages and standard errors obtained from Gaussian fits of distributions showing that the distributions are broader for control and serum-starved cells in comparison to blebbistatin-treated and nocodazole-treated cells.

$\left\langle F F^{*}\right\rangle=4.05 \times 10^{-25} \mathrm{~N}^{2}$. Force fluctuations are reduced by almost an order of magnitude after blebbistatin treatment. The width of the distribution for blebbistatin treated cells was a factor of 2.5 smaller than that of the control cells. For nocodazole treatment, we find no significant differences from the control values, both in amplitude and width of distribution. Serum starvation, in contrast, led to a strong decrease of force fluctuations in comparison to control cells and was comparable to those of blebbistatin-treated cells. However, the distribution of serum-starved cells was twice as broad compared to the blebbistatin treated cells. This shows that the reaction of cells to serum starvation is more variable from cell to cell than the direct inhibition. Overall, a major role of the acto-myosin network in the production of force fluctuations is confirmed by these results. On the other hand, force fluctuations do not depend on the microtubule cytoskeleton.

\section{Force clamp}

Cells are expected to sense external forces and react to them. However, in the experiments so far, we attached the beads to the cell with the force initially adjusted to roughly zero and kept the beads at constant position. Cells exerted contractile forces over time and so the force drifted away from zero slowly in time. To observe cells under a defined constant external force, we constructed a force clamp using feedback (see section 2.2). We measured the force on one of the traps and repositioned the other one to compensate for force changes due to cellular activity. First, we measured cellular force fluctuations at different fixed values of pre-tension. 
Figure 3.8A shows the displacement fluctuations of the two beads attached to cell.
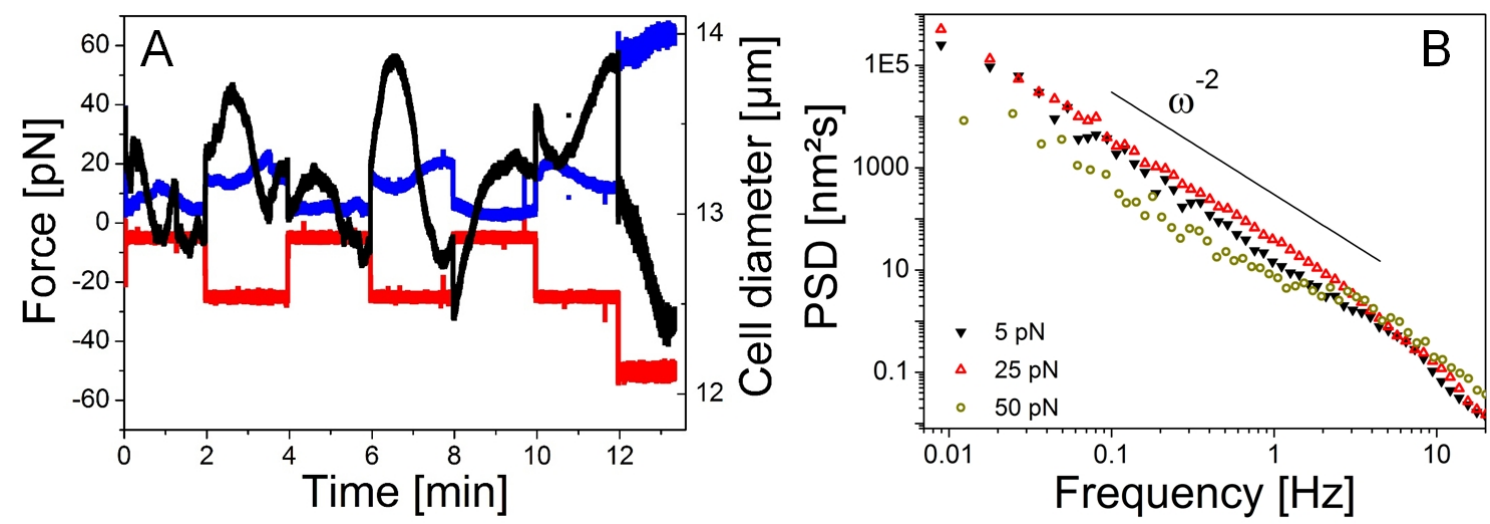

Figure 3.8: Constant force measurement. A) Constant force measurement with switching force between 5 and 25 pN every two minutes. Red and blue data points show forces of trap 1 and 2 (left axis), black data shows the cell diameter (right axis). B) Power spectral densities of the trap position signal at the different clamp forces.

During the measurement, we alternated the pre-tension value between 5 and $25 \mathrm{pN}$, roughly every two minutes. We see an immediate elastic response of the cell to the changing force but no further stereotypic reaction. The cell shows slow irregular fluctuations that are on the scale of minutes. These irregular fluctuations seem to be independent of the direction of the force jumps and also on the total clamp force. We have seen such irregular fluctuations during long-time measurements in constant-position mode (figure 3.9). Similar fluctuations have also been found by others [74]. Panel B in figure 3.8 shows the power spectral densities of the trap

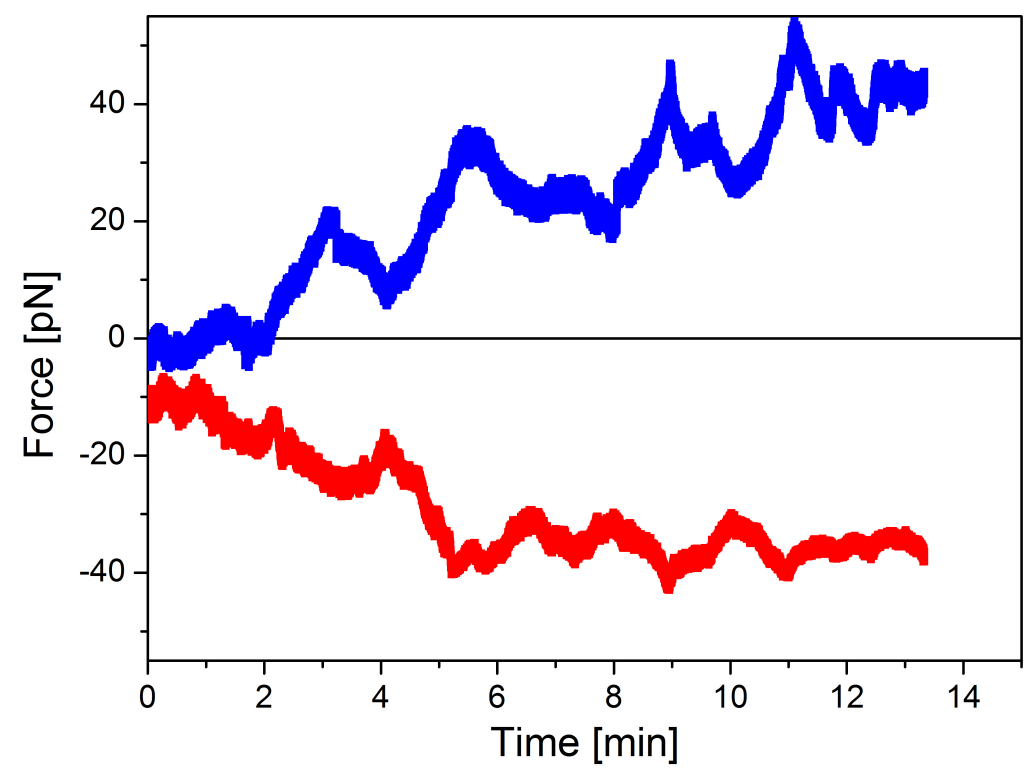

Figure 3.9: Long-time measurement of forces of two attached beads. Periodic fluctuations on top of an overall contraction of the cell at the timescale of the whole experiment are visible.

position signal at the different clamp forces. All spectra follow approximately the same scaling law $\left\langle u_{1}(\omega) u_{2}(\omega)\right\rangle \propto \omega^{-2}$ seen without force clamp and discussed in section 3.3. No significant difference is visible between the different clamp forces $(5$, 25 and $50 \mathrm{pN}$ ). This implies that the strain fluctuations of the cell are independently of the external pre-stress of the optical trap and that therefore the forces that the cell generates to drive its own deformation must be much larger than the maximal 
force that the optical traps are able to exert. With this finding, it is still possible that the stiffness of the cell is in the same range as that of the optical trap.
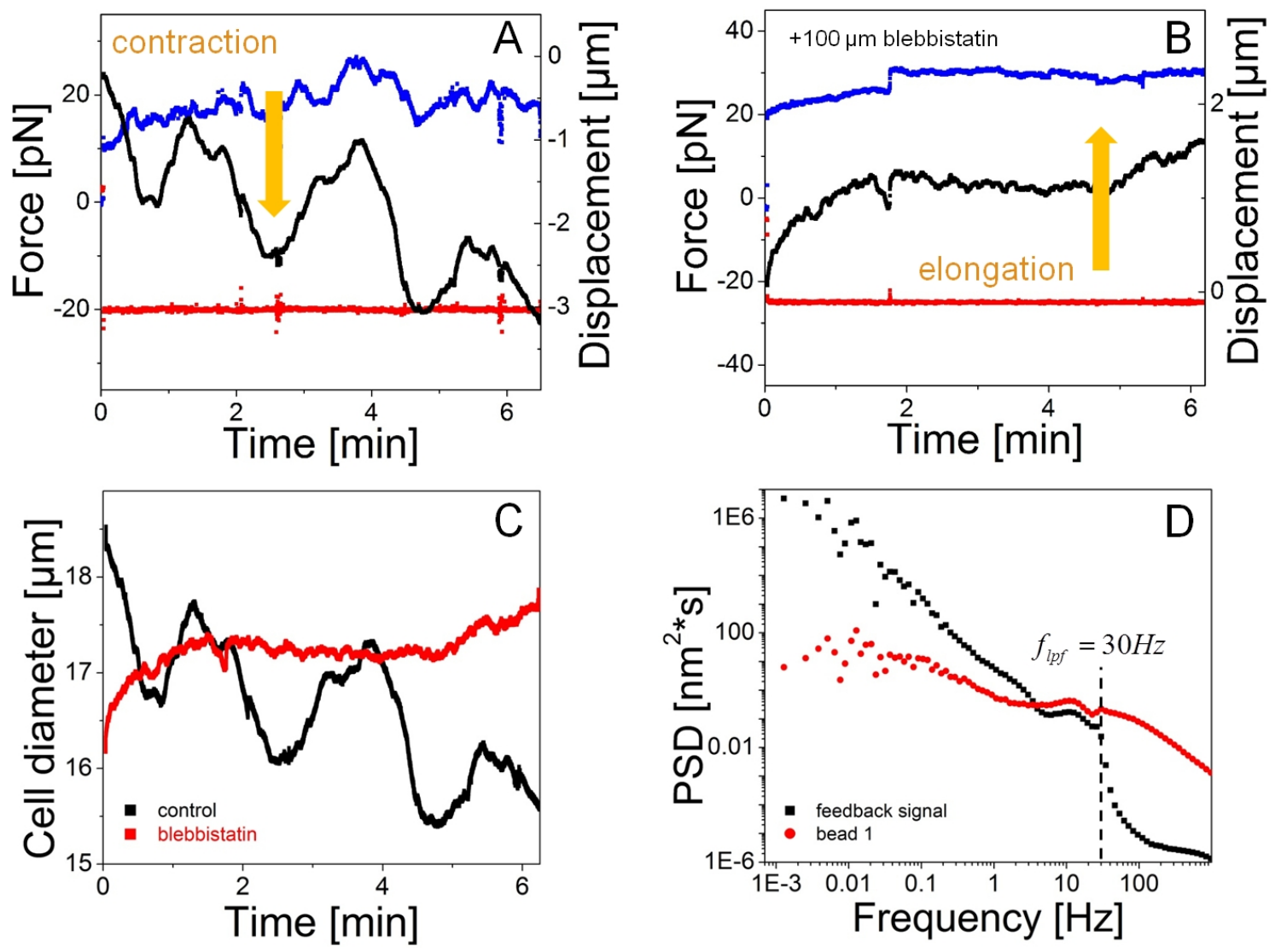

Figure 3.10: Cell deformations under force clamp conditions. A) Measurement at a constant force of $20 \mathrm{pN}$. Force (left-axis) on bead 1 (red) is clamped and bead 2 is moved by AOD. Force on bead 2 (blue) is measured with errors due to trap movement. Black squares show feedbackcontrolled position of trap 2 (right axis). We observe a periodically contracting cell (decreasing and negative numbers of displacement) with increasing amplitude over time. B) Constant force measurement at $25 \mathrm{pN}$ of a blebbistatin-treated cell. Feedback signal shows a slowly elongating cell (larger and positive values of displacement). C) Cell diameter fluctuated and decreased due to contractile forces under external force clamp (black), and the blebbistatin-treated cell showed relaxation behavior and elongation (red). D) Power spectral density of the feedback signal and of displacement of the bead in the stationary trap with a feedback low-pass filter at $f_{l p f}=30$ $\mathrm{Hz}$. Below the filter frequency, all cell movements were followed by the feedback while the relative position of the bead with respect to the trap center remained constant. Above the filter frequency, the feedback does not follow the fluctuations.

We measured long-time response of a cell to an external constant force to test if the cell reacts on longer timescales to this external force. Figure 3.10A shows a measurement in control conditions for approximately six minutes. Force fluctuations and position of the moving trap are recorded at a force of $20 \mathrm{pN}$. The cell showed again an irregular fluctuation, possibly an oscillation similar to the one seen in the long-time constant position experiment. We repeated the measurement under the influence of $100 \mu \mathrm{M}$ blebbistatin to check if the oscillations are driven by the acto-myosin cortex. Figure 3.10B shows the results of the blebbistatin experiment. Blocking the NMM II motors prevented contraction. The blebbistatin-treated cell systematically elongated under the external force by more than $1.5 \mu \mathrm{m}$ after 6 
minutes expected for the creep response of a viscoelastic material. In panel $\mathrm{C}$ of the figure, cell diameters of both cells are plotted over time, emphasizing contracting and expanding control cell and the slowly relaxing blebbistatin-treated cell.

In the feedback, a $30 \mathrm{~Hz}$ low-pass filter is implemented. Figure 3.10D shows the fluctuation spectrum of the error signal of the feedback that is controlling the moving trap and the remaining fluctuation spectrum of the stationary bead. Below the filter frequency, the error signal shows the cellular contractions against internal stiffness also following the $\omega^{-2}$ power-law as expected, whereas the bead fluctuations are at a noise-determined level. Above the filter frequency, the bead is recording the cellular force fluctuations against the now stationary trap.

\section{$3.4 \quad$ Bead size}

Experiments that use tracer beads on the outside of cells are only able to explore an apparent elastic or viscous modulus of the cell because the bead is not fully embedded in the material. The apparent modulus therefore depends on the attachment area and indentation depth of the bead to the cell [75, 76]. To check, how strong our results depend on the bead size and therefore on the attachment geometry, we used bead sizes of 2, 4 and $8 \mu \mathrm{m}$ (figure 3.11) to compare the results of the spring constant and total force generation.
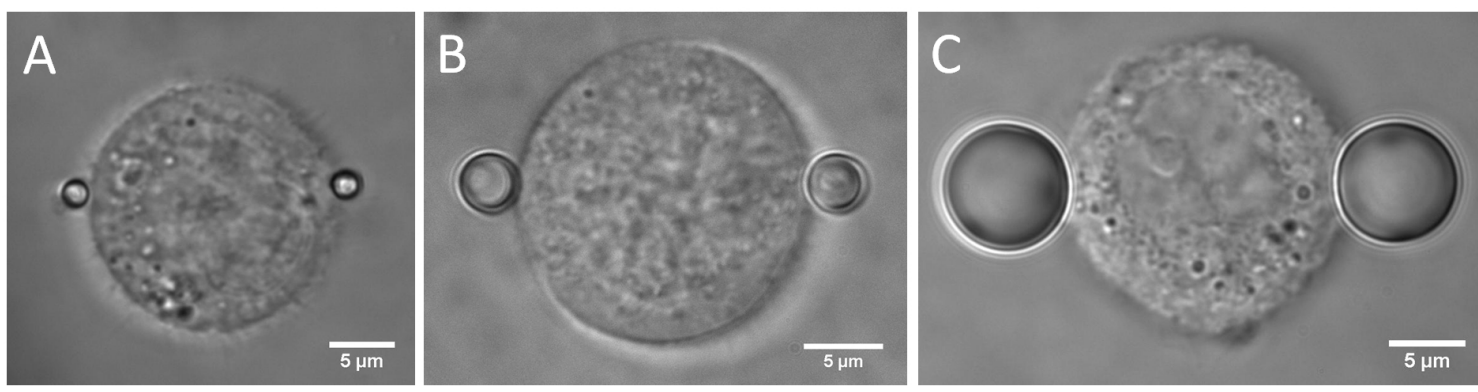

Figure 3.11: Brightfield images of NIH 3 T3 cells suspended between two trapped beads of different bead size. A) $2 \mu \mathrm{m}$ bead. B) $4 \mu \mathrm{m}$ bead. C) $8 \mu \mathrm{m}$ bead.

Figure 3.12 shows the results of the spring constant and total cellular force generation. In all experiments, we see no significant difference between bead sizes of 2 or $8 \mu \mathrm{m}$ compared with our regularly used bead size of $4 \mu \mathrm{m}$. We therefore assume that small differences in the attachment geometry even within one bead size have no significant error contribution to our results. Moreover, using beads of at least $4 \mu \mathrm{m}$ assure us that the cell is able to build focal complexes to attach to the bead [77]. Larger beads allow us to stay away from the cell surface with the optical trapping laser which prevents interactions of the laser light with the cell and would cause errors in the interferometric position detection of the bead. However, to compare the effective spring constant that we measure with a real shear modulus precise knowledge of the cell-bead geometry is needed. 

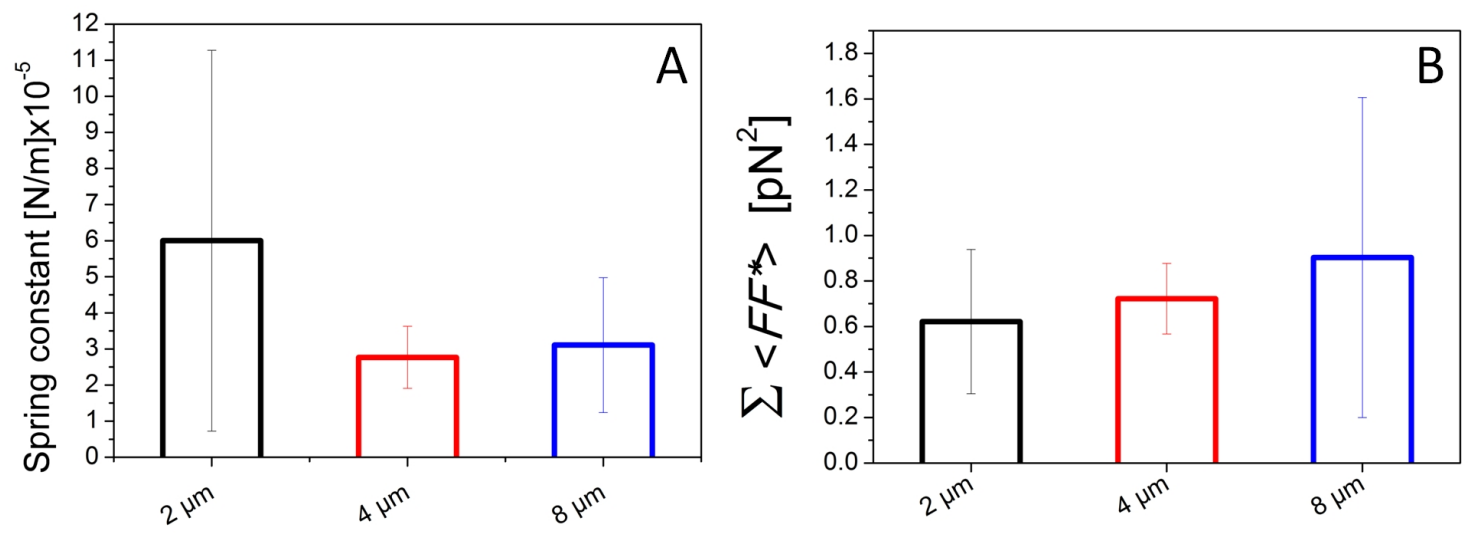

Figure 3.12: spring constant and total force generation of NIH 3T3 cells. A) Effective spring constant of cells with $2(\mathrm{~N}=7), 4(\mathrm{~N}=7)$ and $8 \mu \mathrm{m}(\mathrm{N}=6)$ beads. B) Total force generation of cells with $2(\mathrm{~N}=7), 4(\mathrm{~N}=8)$ and $8 \mu \mathrm{m}(\mathrm{N}=4)$ beads.

\section{Osmotic pressure}

Key player in force generation and elastic stability of a spherical cell is the actomyosin dominated cortex. The mechanical properties of the cell depend on the interplay between tension and viscoelasticity of the cortex, that of the bulk cellular components, and on osmotic pressure. Increasing the osmolarity of the medium (extracellular side) would then result in a collapse of the acto-myosin cortex and shrink the cells [78]. In a preliminary result, we measured the mechanical response of cells exerted to a hyperosmotic shock. We added different amounts of polyethylene glycol

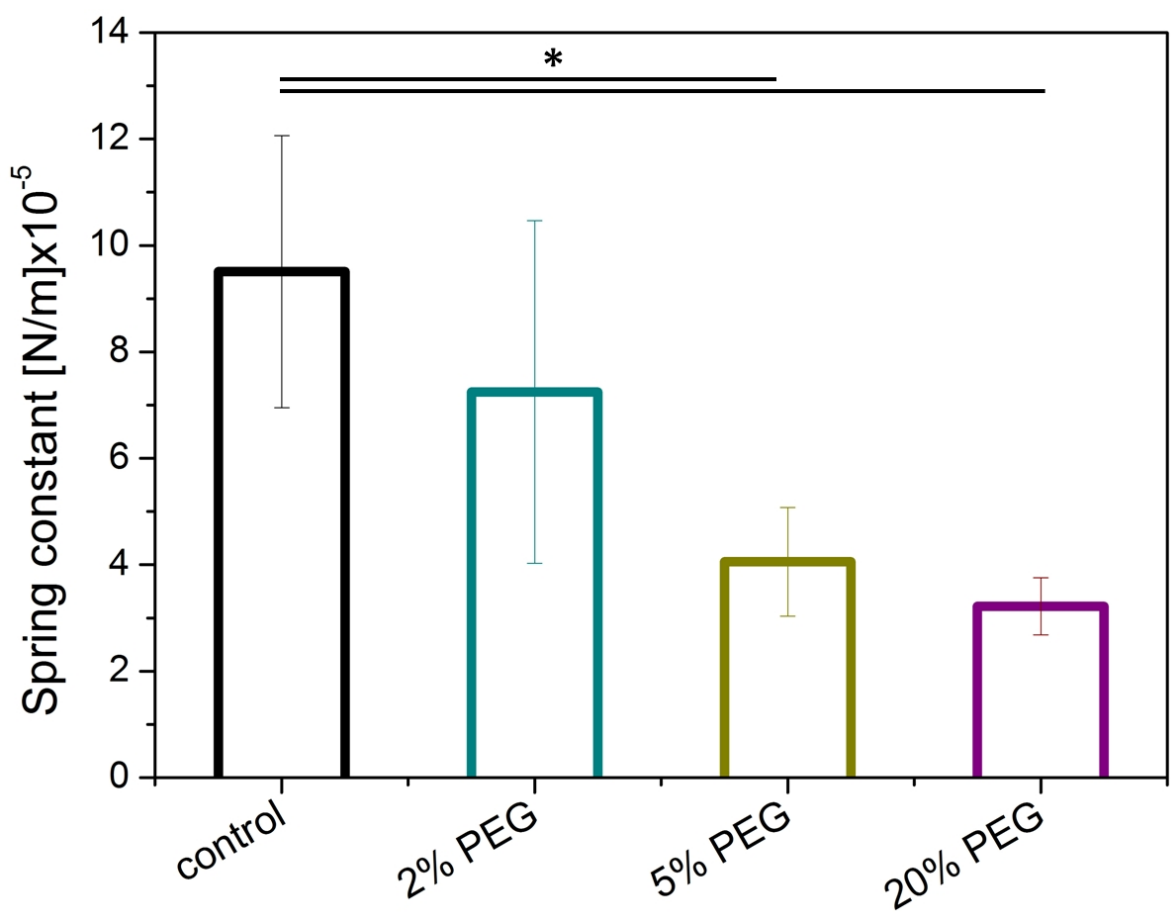

Figure 3.13: Effective spring constant of cells under osmotic pressure. Cells in control conditions and with 2\% (cyan), 5\% (dark yellow) and 20\% (purple) PEG added to the medium. 
(PEG) 300 (Sigma) to the medium and measured the effective spring constants of the cells (figure 3.13). The spring constant of cells in medium with $2 \%$ PEG was $k_{2 \%}=7.24 \pm 3.22 \times 10^{-5}(\mathrm{~N}=5)$, lower compared to the control medium. Adding $5 \%$ and $20 \%$ reduced the mean spring constant further to $k_{5 \%}=4.06 \pm 1.02 \times 10^{-5}$ $(\mathrm{N}=8)$ and $k_{20 \%}=3.22 \pm 0.54 \times 10^{-5}(\mathrm{~N}=3)$, respectively. Although we find no significant differences between the PEG measurements due to the limited sample size, cells treated with $5 \%$ and $20 \%$ were significantly softer than the control cells. Furthermore, the trend of the mean spring constant shows that rounded cells, suspended between two beads, have a lower spring constant with increasing osmotic pressure.

\subsection{Discussion}

Cells constantly communicate with their surroundings. They probe and feel their environment using mechanical cues which they exchange with their neighbors in tissue through direct connections or extracellular matrix. We showed that the cortical acto-myosin network is the essential player determining the mechanics of suspended cells. Control experiments revealed that the lipid envelope of the cell provides no major contributions to the overall elasticity of a fibroblast cell. The spring constant of an osmotically stretched GUV was even lower than that of cells with reduced cortical tension by blebbistatin treatment or under serum-free conditions. To relate the spring constant of the cell $k_{\text {cell }}=9.5 \pm 2.5 \times 10^{-5}$ to material properties, one needs to take geometrical factors and the strong volume constraint due to osmotic pressure into account. Assuming the cell as an elastic solid, the spring constant can be related to an effective Young's modulus E of approximately $100 \mathrm{~Pa}$ [40], comparable to results from atomic force microscopy (AFM) and optical trapping for small deformations [79], making similarly naive assumptions. However, this value is rather descriptive in nature as one need to take geometry and material constants of the cellular components into account to be related quantitatively to a cell material constant.

According to the generally accepted wisdom that actin is dominating the elastic response of the cells $[79,80]$, we found that depolymerizing microtubules had no significant effect on cell stiffness. The effect that microtubules embedded in an elastic matrix can bear orders of magnitude higher compressional forces than bare microtubules [81] seemed not to play a role in our experimental geometry. The importance of NMM II has been shown by the fact that blebbistatin treatment leads to a roughly threefold decrease in effective cellular stiffness. Future experiments will allow the dissection of the distinct contributions of different myosin motors to cortical tension and force production. Especially myosin I is known to contribute to cortical tension in cells [82]. An effect that could not be tested here, since blebbistatin is highly specific for myosin II [83]. Stiffening in response to internal stress is, indeed, a characteristic property of semiflexible polymer networks and has been seen in experiments with model networks before [56, 84]. Interestingly, it was found recently that blebbistatin-treated cells suspended in an optical stretcher not adher- 
ent to any substrate are stiffening [85], possibly pointing to nonlinear effects or to a role of integrin-mediated adhesion domains for the contractile acto-myosin cortex.

Stress fluctuations indicated the molecular origin of cortical tension. Myosin seemed to be the responsible molecular motor as indicated by the blebbistatin experiments. The $\omega^{-2}$ power law in the force PSD is a signature expected at shorter times than the characteristic attachment time of the motors [84]. The $\omega^{-2}$ scaling law has also been observed in experiments with probe particles embedded in cells $[52,86]$ and in cytoskeletal model networks [56] and NMM II minifilaments are likely to produce holding times on the order of $10 \mathrm{~s}$. However, these reports of characteristic myosin force production times cannot explain the observed oscillation periods as they are one order of magnitude lower. We therefore think that more complex and collective effects of cellular force generation and transduction cause the observed slow shape fluctuations.

AFM experiments showed that cells adhering to a two-dimensional substrate generate higher stresses at higher temperatures [87] which is in contrast to our finding that the force fluctuation amplitude is independent of temperature. Different behavior is not unexpected, though, since the active processes driving cell spreading are clearly different from those creating fluctuations of the rounded up cells. The lower amplitude force fluctuations in serum-free medium are consistent with a report that serum is necessary to fully phosphorylate the myosin II regulatory light chain [88]. 



\section{Construction of a confocal trap}

One of the goals of this thesis was to investigate the active and mechanical properties of cells that are suspended between two optically trapped beads and to image the structure and distribution of their cytoskeletal filaments. Cell rheology experiments were done with the trapping setup described previously, which is also equipped with a green laser for epifluorescence imaging of RFP-tagged microtubules [89]. For imaging of LifeAct transfected cells, a blue laser (Sapphire, Coherent) was coupled into the illumination path with a dichroic mirror (AHF analysetechnik, Tübingen, Germany). Preliminary experiments using this setup showed that it was not possible to see single structures in transfected cells using epifluorescence illumination (figure 4.1).
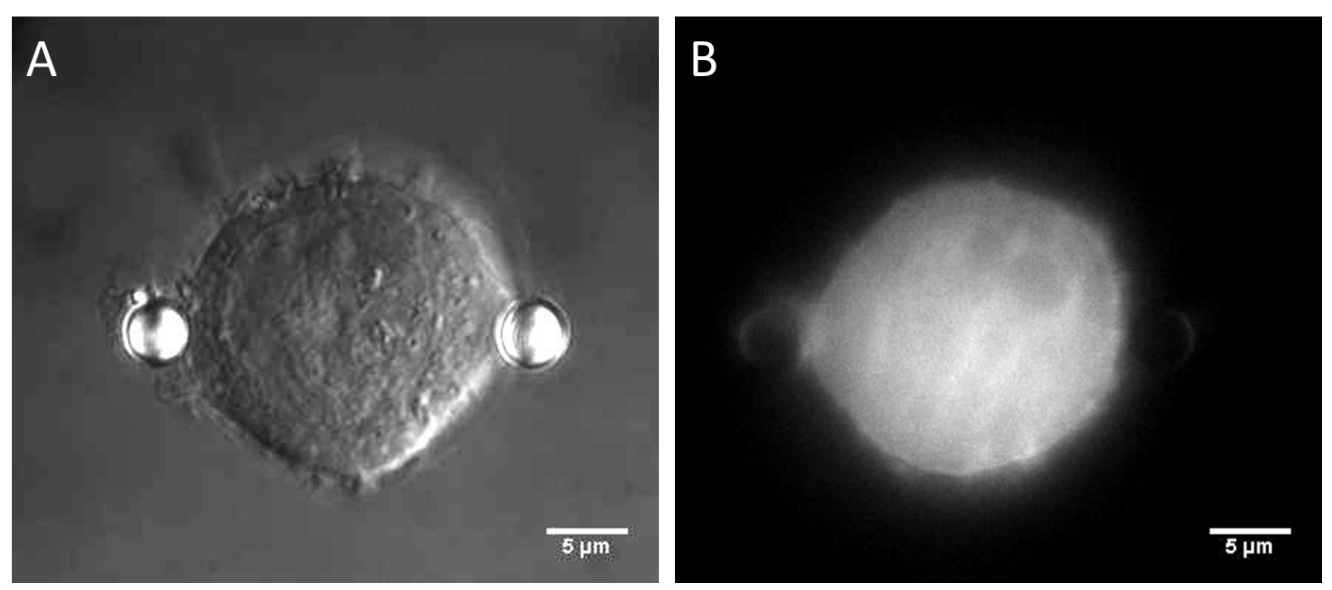

Figure 4.1: GFP-Actin transfected cell (A) DIC brightfield image (B) fluorescence image

The main reason for this is that in this setup configuration light from the whole illuminated focal volume is collected and imaged. Imaging at a round, transfected fibroblast cell in solution, which has a diameter of $\approx 20 \mu \mathrm{m}$, generated a large amount of background fluorescence, making it impossible to visualize details. One option to overcome this problem is to use a confocal microscope. Confocal microscopy allows for the collection of light only from the focal plane, with scattered light from other planes blocked by a confocal pinhole. 


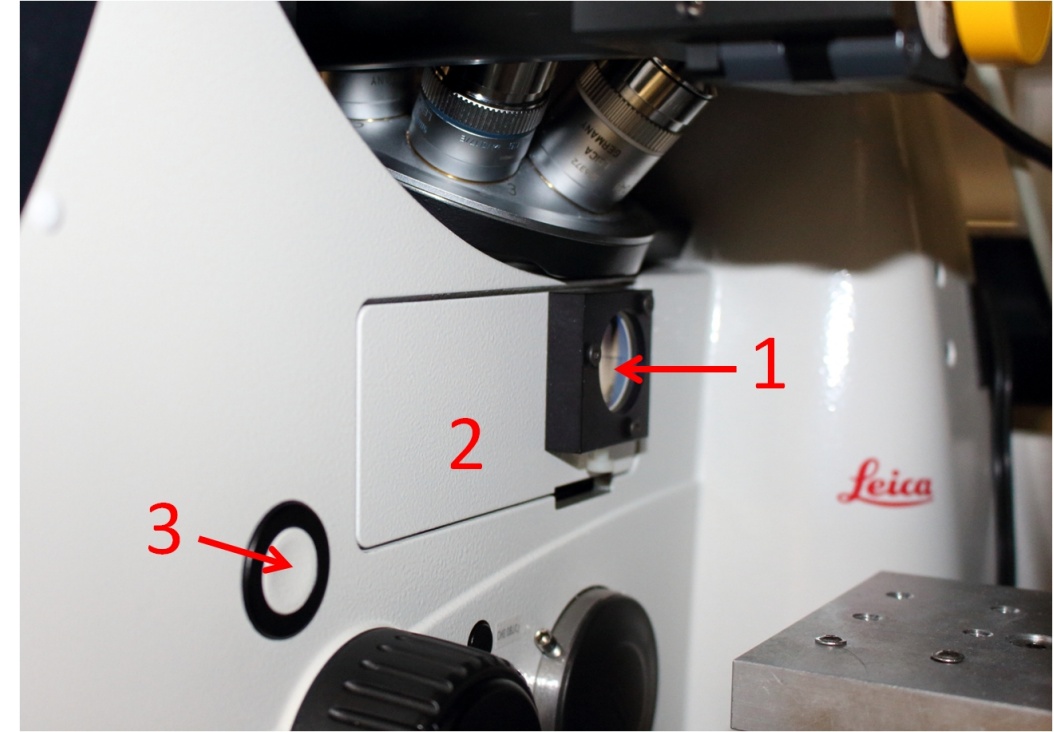

Figure 4.2: Coupling port for the trapping laser with mounted telescope lens (1). Behind the cover (2) is the filtercube turret for epifluorescence filters. 3: Button to open filtercube port.

\subsection{Confocal trap}

In our setup, we use a Leica SP5-X confocal microscope (Leica, Wetzlar) to image the samples. Since the microscope is not designed to implement an optical trap, certain limits and drawbacks have to be overcome. First, a suitable optical port has to be found, to couple the trapping laser into the light path of the microscope and focus it into the back-focal plane of the objective. The microscope has only two ports for external light sources, one for the white-light scanning laser and one for an epifluorescence mercury bulb. Since it is not suitable to remove one of the light sources or to couple the trapping laser into one of these light paths, we decided to use a camera port which is supposed to be used for a two-photon illumination camera. This port is situated on the right side of the microscope, directly next to the filtercube turret for fluorescence illumination (see fig. 4.2).

First, a redesigned filtercube was placed into the filter turret. It has a $90^{\circ}$ rotated design, so that the trapping laser is reflected coming from the right side upward into the objective, in contrast to the regular design which reflects light from a mercury bulb coming from a port at the backside of the microscope upward. Due to the design of the filter turret, the filter port next to the trapping dichroic has to be empty. Otherwise the trapping laser would be blocked by the neighboring cube (figure 4.3).

The dichroic mirror has to transmit all wavelengths that are necessary for confocal imaging (405 $\mathrm{nm}$ from the diode laser and 470-670 $\mathrm{nm}$ from the white-light laser) and to reflect the $1064 \mathrm{~nm}$ light from the trapping laser. We used a mirror that has a high transmission for wavelengths below $700 \mathrm{~nm}$ and reflects wavelengths above $700 \mathrm{~nm}$ (T700 DCSPXR-UV, AHF analysetechnik). 


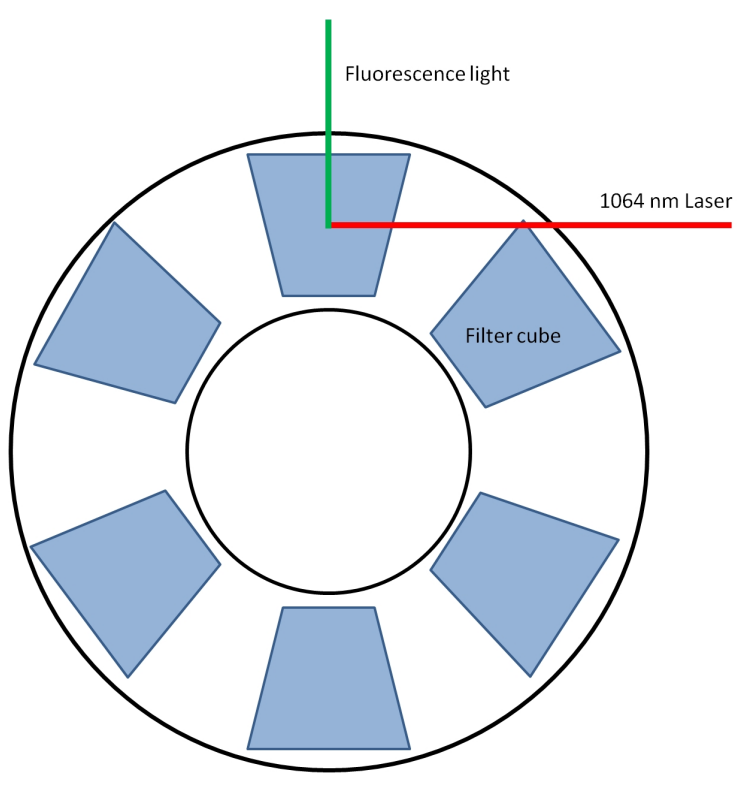

Figure 4.3: Schematic view of the filtercube wheel. Six filtercubes sit in the filtercube wheel. The fluorescence light comes from the top and is reflected upwards into the objective. For the trapping dichroic, the cube is rotated by $90^{\circ}$. The trapping laser comes from the right and is reflected upwards. The filter position on the right side next to the trapping dichroic has to be empty to allow undisturbed passage of the laser light.

\section{Dual optical trap}

A 1064 nm laser (Compass, Nd:IVO 4 , 4W, Coherent Inc., Santa Clara, CA, USA) was used for optical trapping due to the low absorption rate of water at this wavelength. The laser is protected from back reflections with an optical isolator (IO5-1064-VHP, Thorlabs, Newton, NJ, USA). To overfill the objective, the laser is expanded with a beam expander (4401-359-000-20, Qioptiq Photonics, Göttingen, Germany) and brought onto a breadboard raised to the height of the microscope port with a periscope and two mirrors. To achieve maximum reflection with the periscope, the polarization of the laser beam is adjusted with a $\lambda / 2$-plate (WHP05M1064, Thorlabs) (figure 4.4).

On the breadboard, the laser passes through a combination of a $\lambda / 2$-plate in a motorized rotation stage (CLR1/M, Thorlabs) and a polarizer (GL10-C26, Thorlabs) which allow adjustment of the trapping power in the sample plane without changing the laser power. To create two individual steerable traps, the laser is then

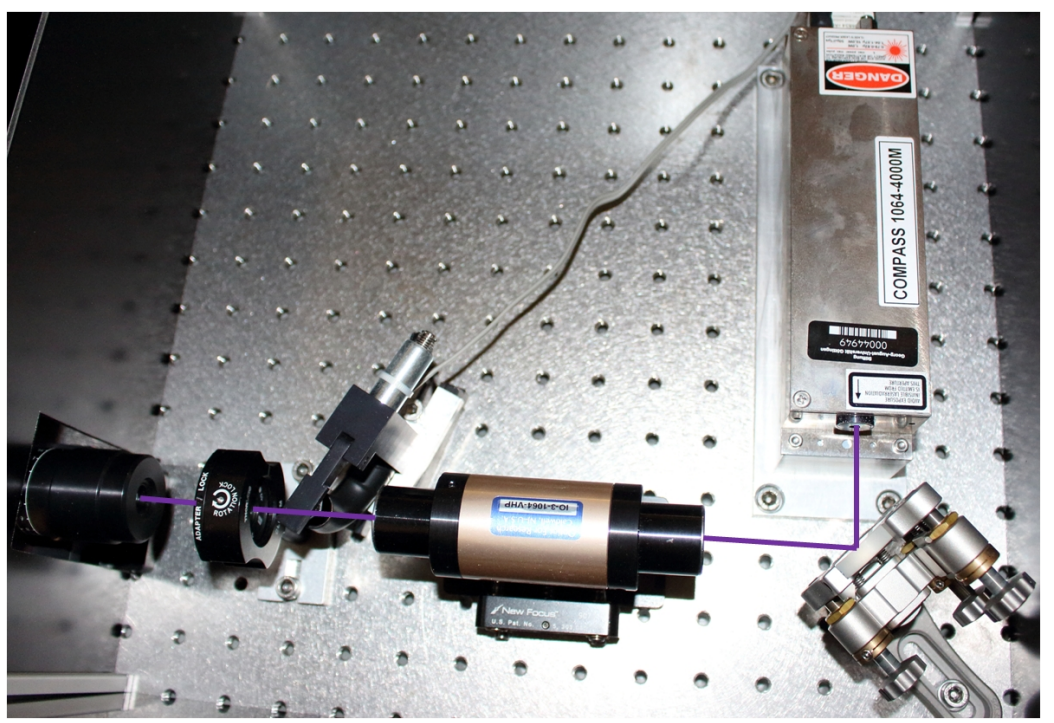

Figure 4.4: Trapping laser. The laser beam is protected from backreflections with an optical isolator, polarized and expanded with a beam expander 


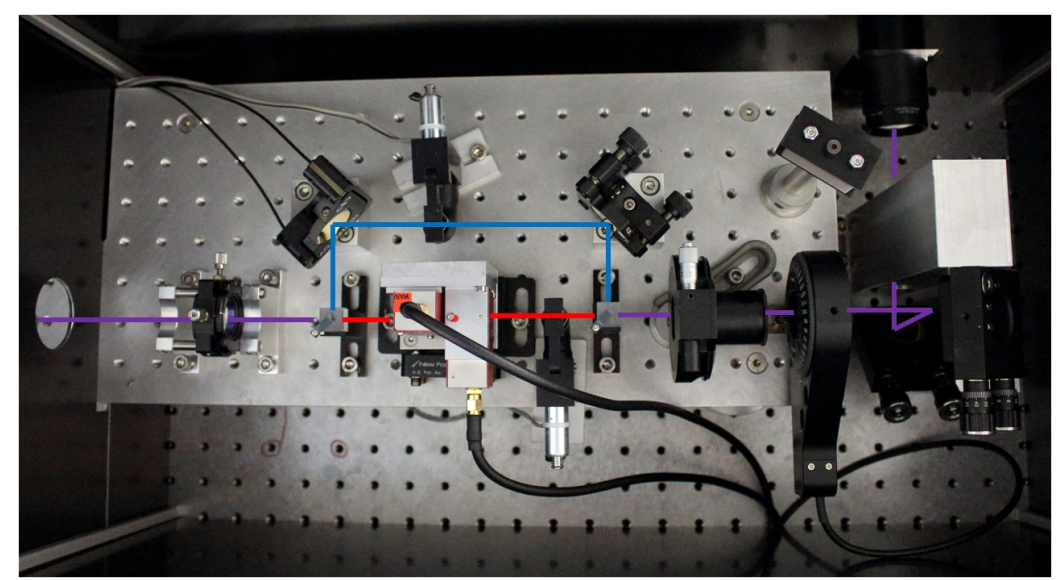

Figure 4.5: Photograph of the beam path on the breadboard. The trapping laser exits the beam expander on the top-right side of the photo. It is raised to the breadbord with a periscope and polarized with a $\lambda / 2$ plate. It is then split into the two individual steerable traps. After recombination of the two beams it is focused by telescope lenses into the backfocal plane of the objective.

split with a Glan-Laser polarizing beamsplitter into two beams with orthogonal polarization. In one beam path, the beam passes a pair of AODs for fast steering in the x-y direction (DTSXY-400-1064, AA Optoelectronics, Orsay, France). Both AODs are steered by voltage controlled oscillators (VCO, DRFA10y, AA Optoelectronics). The other beam is reflected by a mirror and a piezoelectric mirror (AG-100N, Newport) for $x-y$-steering of the trap, then recombined with the first beam via a second polarizing beamsplitter. Both beams are then coupled by a pair of telescope lenses (1:1.6, G322-308-525 and G322-352-525, both Qioptiq Photonics) and the previously described dichroic mirror into the back-focal plane of the objective of the microscope (100x, 1.4 NA Oil CS2, Leica) which focuses the beam into the sample plane. A photo of the components on the breadboard is shown in figure 4.5.

The sample is mounted on a 3D translational stage which can be steered with micrometer screws and piezo elements and is controlled by the microscope software. To align the laser into the objective, a tube that can be screwed into the objective revolver is very helpful. One can place an adjustment laser (Thorlabs) onto the tube and focus it into the outlet of the $1064 \mathrm{~nm}$ laser. In a second step, a pinhole can be placed on top of the tube and the $1064 \mathrm{~nm}$ laser can be aligned so that it passes through that pinhole. Fine adjustments are then made using the backreflected diffraction pattern from a glass-water interface of a mounted sample which is imaged with the bright-field camera (Cool-Snap EZ, Photometrics, Tucson, AZ, USA).

\section{Back-focal plane detection}

To detect trapping laser light after passing the sample, certain custom-made adjustments had to be made to the condenser mount. Two attachment plates were fixed to the condenser mount to balance the different heights. The second plate is equipped to hold a dichroic mirror and has drilled holes to hold poles for the Thorlabs microcage system that includes the back-focal plane detection system. Everything needed for the detection path is then added to the cage system (figure 4.6). 


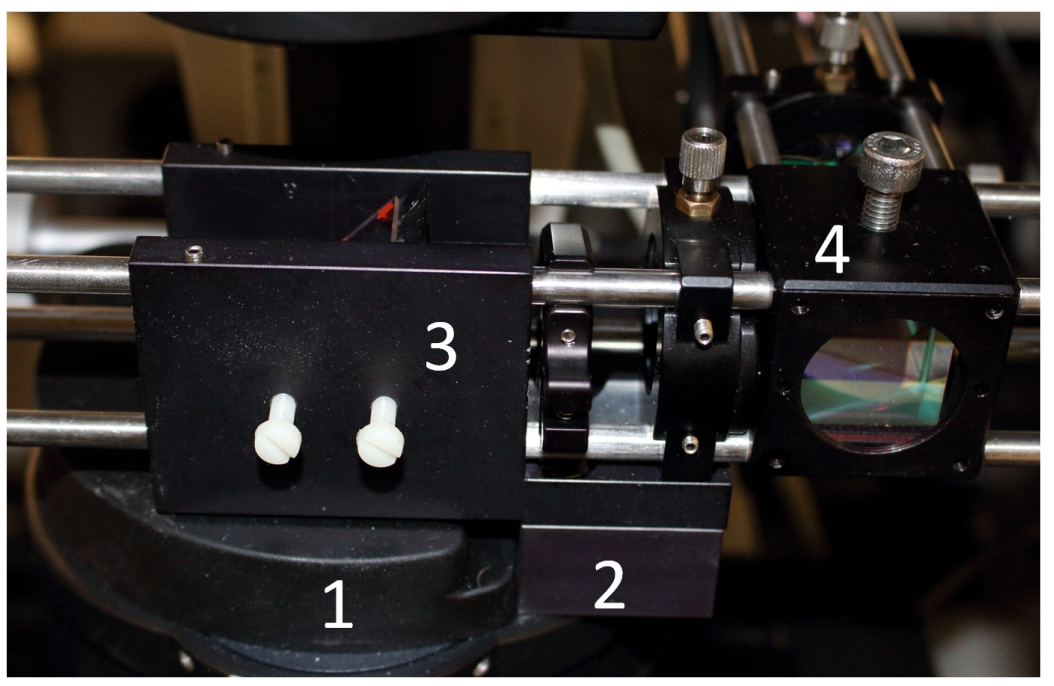

Figure 4.6: Attachment of the detection path to the microscope. 1: Condenser mount. 2: First attachment plate connected to the condenser mount. 3: Second attachment plate mounted to the first plate with a dichroic mirror to decouple the trapping laser and openings for the cage system. The trapping laser is reflected to the right and split up into the orthogonal polarizations by a polarizer (4) onto the QPDs.

The laser light from the traps is collected by the condenser (S01, 1.4 NA Oil, Leica). The trapping light is reflected by a dichroic mirror and made parallel with a second lens (G313 217 329, Qioptic). With a mount for putting neutral density filters into the beam line, the laser power can be adjusted to fit to the power range of the QPDs. The beam is split up with a polarizing beamsplitter (PBS 253, Thorlabs) into two orthogonally polarized beams. The back-focal plane of the condenser is then imaged by a lens (LB 1596-C, Thorlabs) onto a QPD (SPOT9-YAG, 10mm diameter, OSI Optoelectronics, Hawthorne, CA, USA). Before each QPD a laser line filter (FL1064-10, Thorlabs) is inserted which only passes trapping light, to reduce noise. The QPD signals are digitized and read out using an FPGA (NI PCI-7833R, National Instruments) and the LabVIEW software described for the other setup (section 2.1.4) A schematic view of the setup is shown in figure 4.7.

\section{Characterization of the trap}

To characterize the confocal trap and test it functionality, we performed several control measurements. To align the traps in the sample plane, the back reflections of the trapping laser at the bottom glass-water interface of a sample are imaged with the bright-field camera. The reflections should be roughly in the center of the field of view and symmetric. The intensity distribution should not shift from one side to the other during focusing. If this is not the case, the beam does not enter the objective perpendicular to its aperture plane. In figure 4.8 the reflections of both traps are shown.

The trap has to be combined with confocal imaging, therefore we have to make sure that the trapping light is not blocked during image acquisition. Therefore, the place in the filter-wheel where the trapping dichroic mirror is located has to be set to empty in the microscope software, otherwise it will rotate the filter-wheel to an 


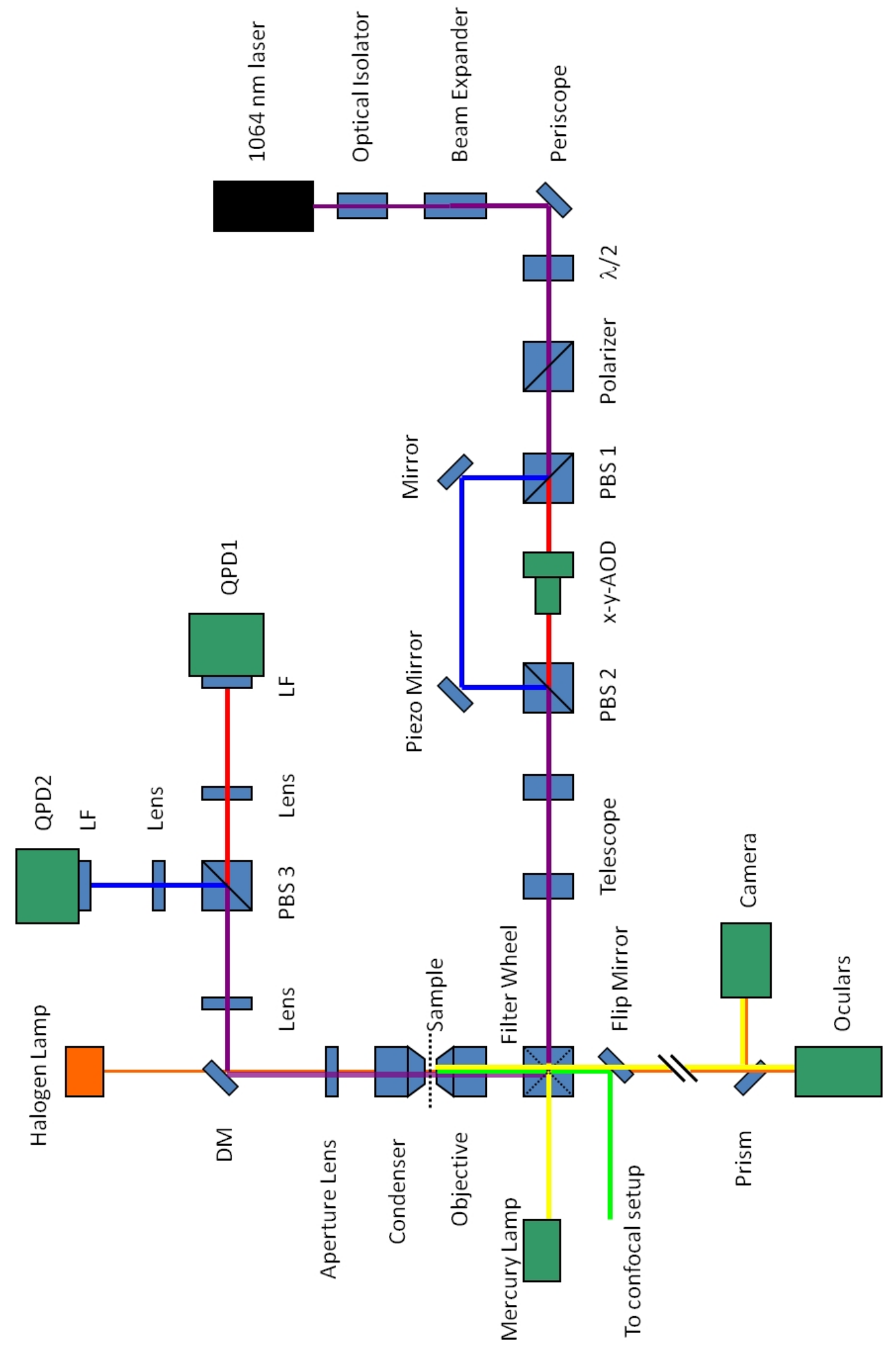

Figure 4.7: Schematic view of the confocal trap. A $1064 \mathrm{~nm}$ laser is split into two beams with orthogonal polarization and coupled into the Leica SP5 X confocal microscope. One beam is steerable with a pair of AODs and the other beam can be steered with a piezo driven mirror. The light of the beams is collected by a condenser and the back-focal plane of each beam of the condenser is imaged onto separate QPDs for detection. PBS: polarizing beam splitter, DM: dichroic mirror, LF: laser-line filter 

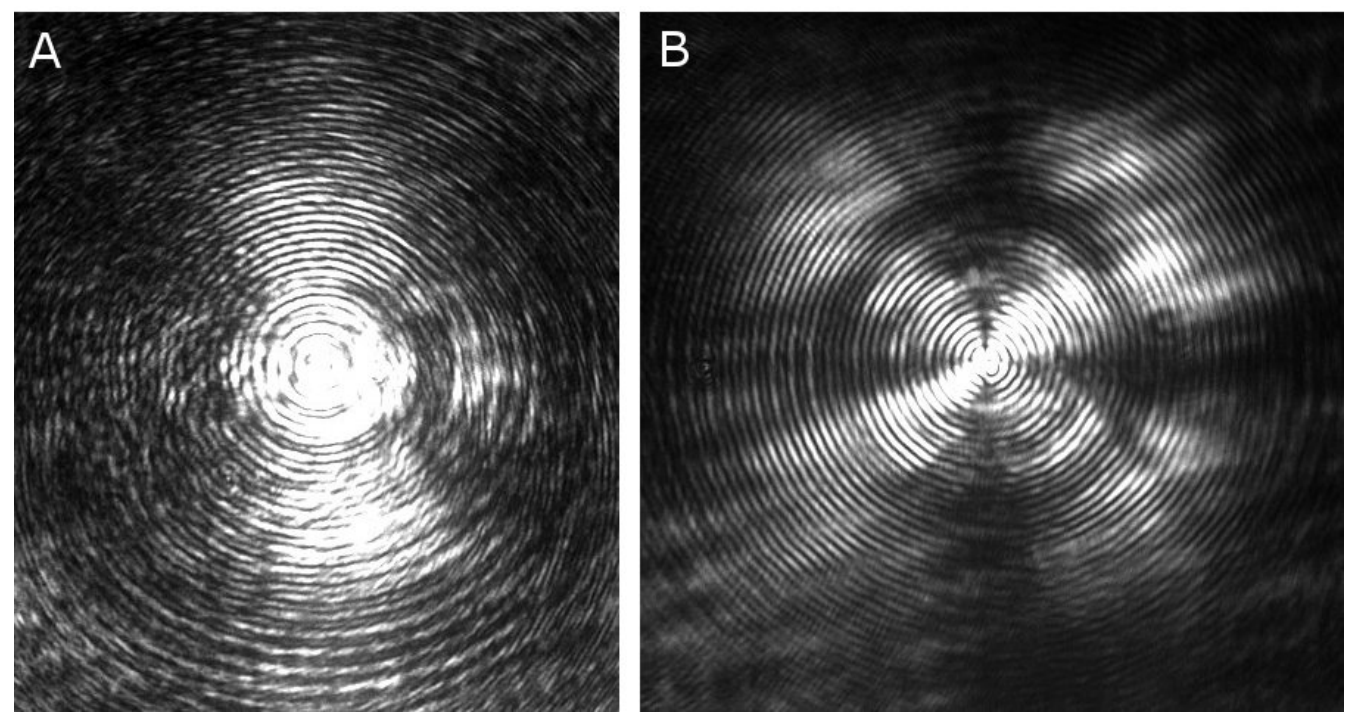

Figure 4.8: Trapping laser reflections of the bottom glass-water interface. The different patterns are due to the different polarizations of the beams. A) reflection of the direct beam, B) reflection of the indirect beam

empty position for imaging. As a test, one $4.95 \mu \mathrm{m}$ fluorescent bead $(660 / 690 \mathrm{~nm}$, FC05F/11376, Bangs labs, Fishers, IN, USA) was trapped in each trap and imaged using the confocal software (figure 4.9).

To test the performance of the setup, recordings of the noise level were made. The QPD signal from the optical trap with a trapped $4 \mu \mathrm{m}$ polystyrene bead was recorded and compared with the signal from an empty trap. The QPD signal was recorded for roughly 11 minutes with a sampling frequency of $5 \mathrm{kHz}$. The power spectral densities for both measurements are shown in figure 4.10. The corner frequency of the trapped bead is around $100 \mathrm{~Hz}$. At higher frequencies, the spectrum decays with the typical $\omega^{-2}$ scaling (see figure 2.3). At intermediate frequencies, the plateau regime for the confined bead can be seen. At frequencies below $0.2 \mathrm{~Hz}$ noise with roughly a scaling of $\omega^{-1}$ adds to the power spectrum. The blue curve of the graph shows the spectrum without the bead. The amplitude of the fluctuations is much lower compared to the spectrum with a bead at high frequencies. At frequencies below $0.2 \mathrm{~Hz}$, both spectra show the same behavior. In this regime the spectrum is noise-dominated. The noise probably comes from beam-pointing fluctuations as has been shown in other setups with the same laser [90]. Other sources of noise may be present in the low frequency regime, as the scaling of the power spectrum

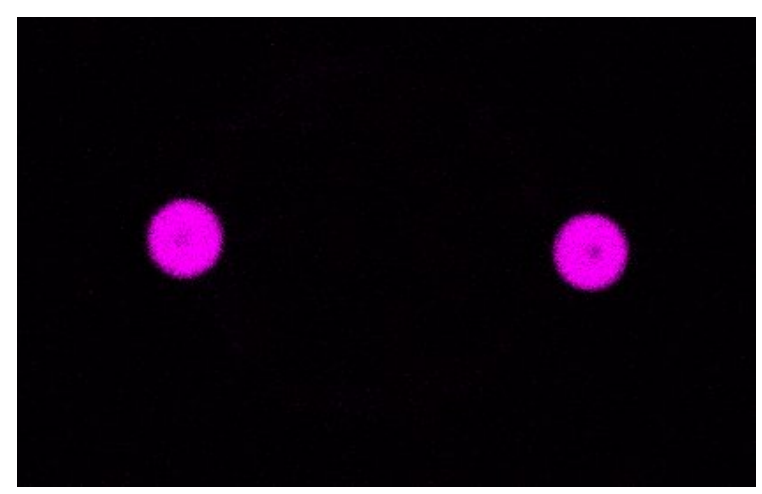

Figure 4.9: Confocal image of $4.95 \mu \mathrm{m}$ fluorescent beads in the optical traps (excitation/emission: $660 / 690 \mathrm{~nm})$. 


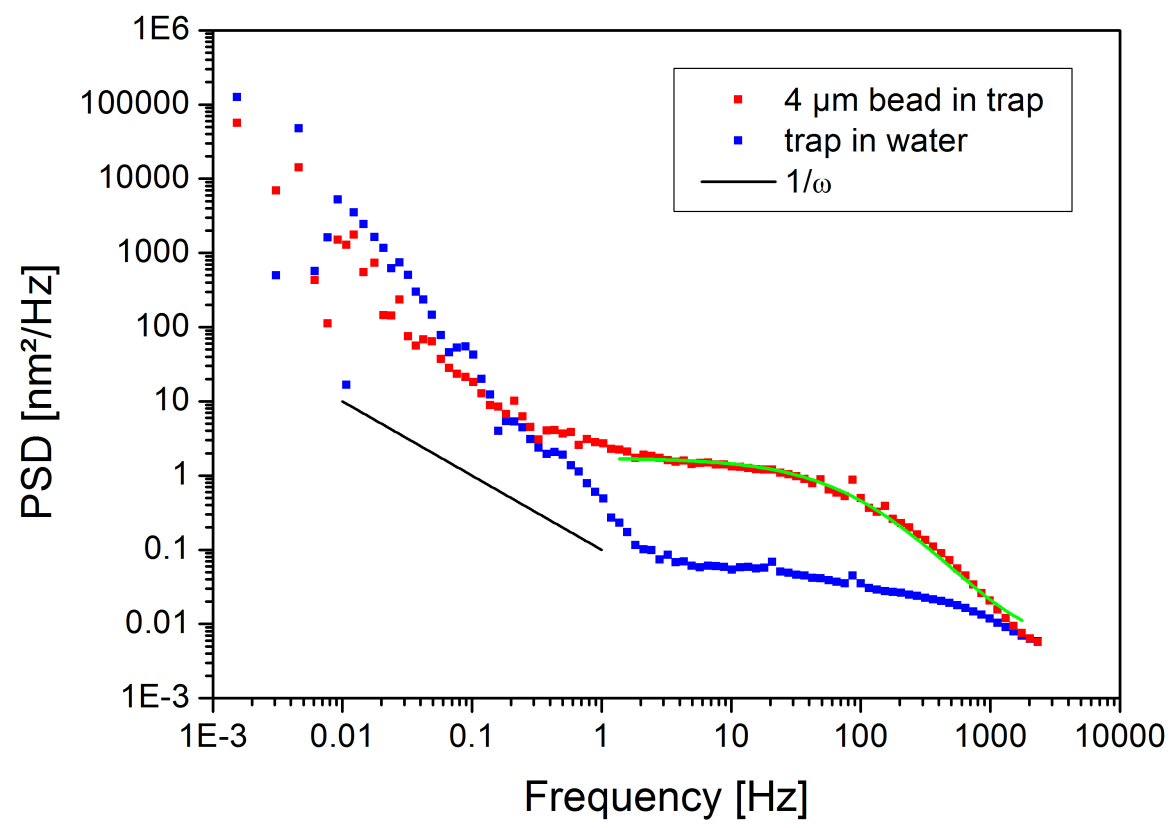

Figure 4.10: Power spectral densities of a $4 \mu \mathrm{m}$ bead trapped in water (red) with a Lorentz fit (green), and of the empty trap in water (blue). The black line indicates $\omega^{-1}$ scaling.

in this range is steeper than the expected $\omega^{-1}$ scaling. One source of noise might be mechanical vibrations due to the rather unstable attachment of the detection path to the condenser mount, which is only attached to the lever of the microscope body at one point. This attachment makes it prone to disturbances and torque.

\subsection{Fibroblasts in the confocal trap}

In chapter 3, we measured the force fluctuations and elastic properties of suspended cells and found that both properties are highly dependent on the acto-myosin network. It is interesting to explore, how the acto-myosin network changes in the cell corresponding to the actual force production. With the construction of the confocal trap, we have the right tool, to combine force measurements with imaging of transfected cells showing the distribution of actin fibers or myosin II motorproteins.

We successfully transfected NIH 3T3 fibroblast cells with LifeAct-RFP and NMM II-GFP to visualize filamentous actin and myosin (see section 2.5). Figure 4.11 shows a confocal slice of the middle plane of a NIH 3T3 fibroblast cell lying on a pluronic coated coverslip. We see that actin and myosin are mainly located in the cellular cortex. Most of the space is covered by the nucleus and there are a few agglomerations of NMM II.

For in-situ imaging of the trapped beads and the cell, we used fluorescently labeled beads (figure 4.9). These beads fluoresce in the far-red wavelength regime 

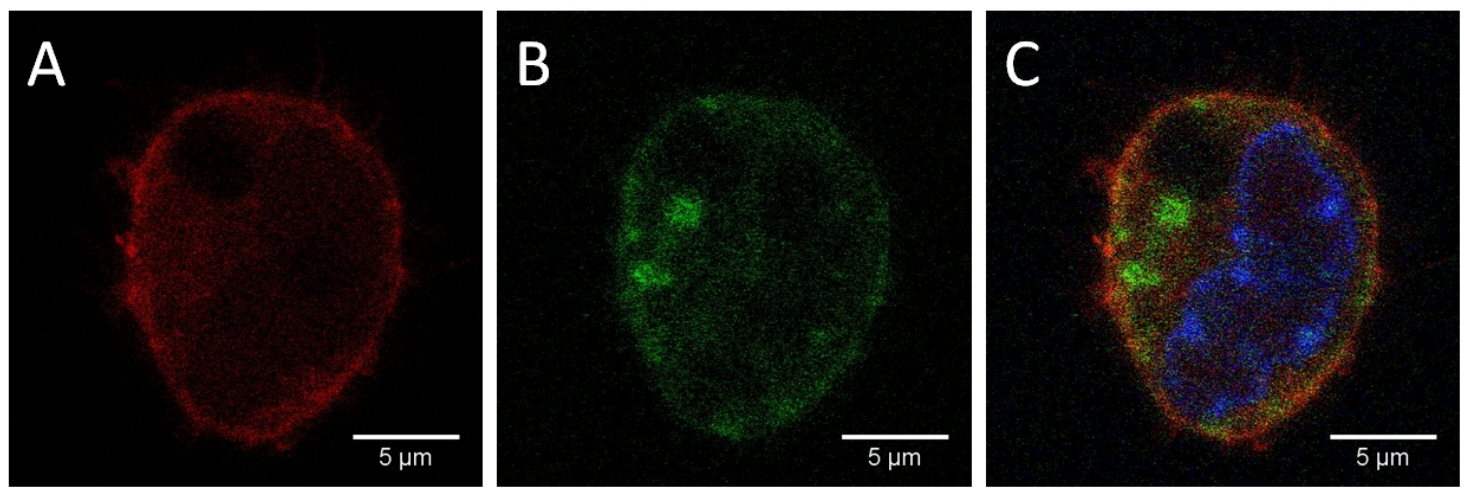

Figure 4.11: Confocal slice of the middle plane of a NIH 3T3 fibroblast. A) LifeAct-RFP staining for actin. B) non-muscle myosin II-GFP C) merge of A and B and Hoechst staining for nucleus (blue).

(690 nm) and do not interfere with commonly used fluorescent tags in cell imaging (TRITC, RFP). Therefore they are an ideal choice for a combination of optical trapping and confocal imaging. Since trapping laser and confocal imaging laser are both focused by the objective they have identical focal planes move together in the zdirection. Therefore, it is not possible to do z-stack recordings of the cells while they are trapped, yet. For a 3D image of the whole cell, they have to be released from the trap and deposited on the surface. Figure 4.12A and B show brightfield and confocal fluorescent images of a fibroblast of the imaging plane, which is defined by the two trapped beads. Part $\mathrm{C}$ of the image shows a $\mathrm{X}-\mathrm{z}$ maximum intensity projection after the cell is deposited on the pluoronic coated coverslip to prevent adhesion. Here, the actin distribution of the cell looks quite different from the previous one (figure 4.11), although both cells were imaged at the same conditions. In the first cell, actin is only present in the cortex. In the second cell, however, actin fibers span the whole cell with centers of agglomeration, where several fibers seem to be connected. In the myosin II channel we see again patches of agglomeration of motor proteins similar to the cell in image 4.11 .

Already these two images show the highly diverse distribution of actin in the cells. This diversity makes it hard to look for changes in the actin distribution of cells that might correspond with changes in the force production. Figure 4.13 shows a collection of confocal images of fibroblast cells trapped between two beads. All of these cells were imaged in control medium at room temperature. Major differences can be seen in the distribution of actin fibers. There are cells where only the cortex is visible and almost no actin structure can be seen inside the cell (panel A and D). Some cells have a diffuse background distribution of actin in the cytosol besides the well defined cortex (panel B and E), whereas other cells show a clear structure of filamentous actin also in the cytosol (E, F, G) or even a defined actin ring around the dark nucleus (E). At some cells, the cortex is rather flat (A, B, C), in other cells actin filaments or filopodia are sticking out from the actin cortex (D, E, G). One cell shows a structure that looks like blebbing $(\mathrm{H})$ which might be a sign for apoptosis [91] or a switch in migration behavior [92]. Nevertheless, this images show the large cell-to-cell variation of the distribution of filamentous actin in a rounded-up state.

To investigate changes in the actin structure of a single cell over time which can be related to changes in its force production, we recorded confocal images and 


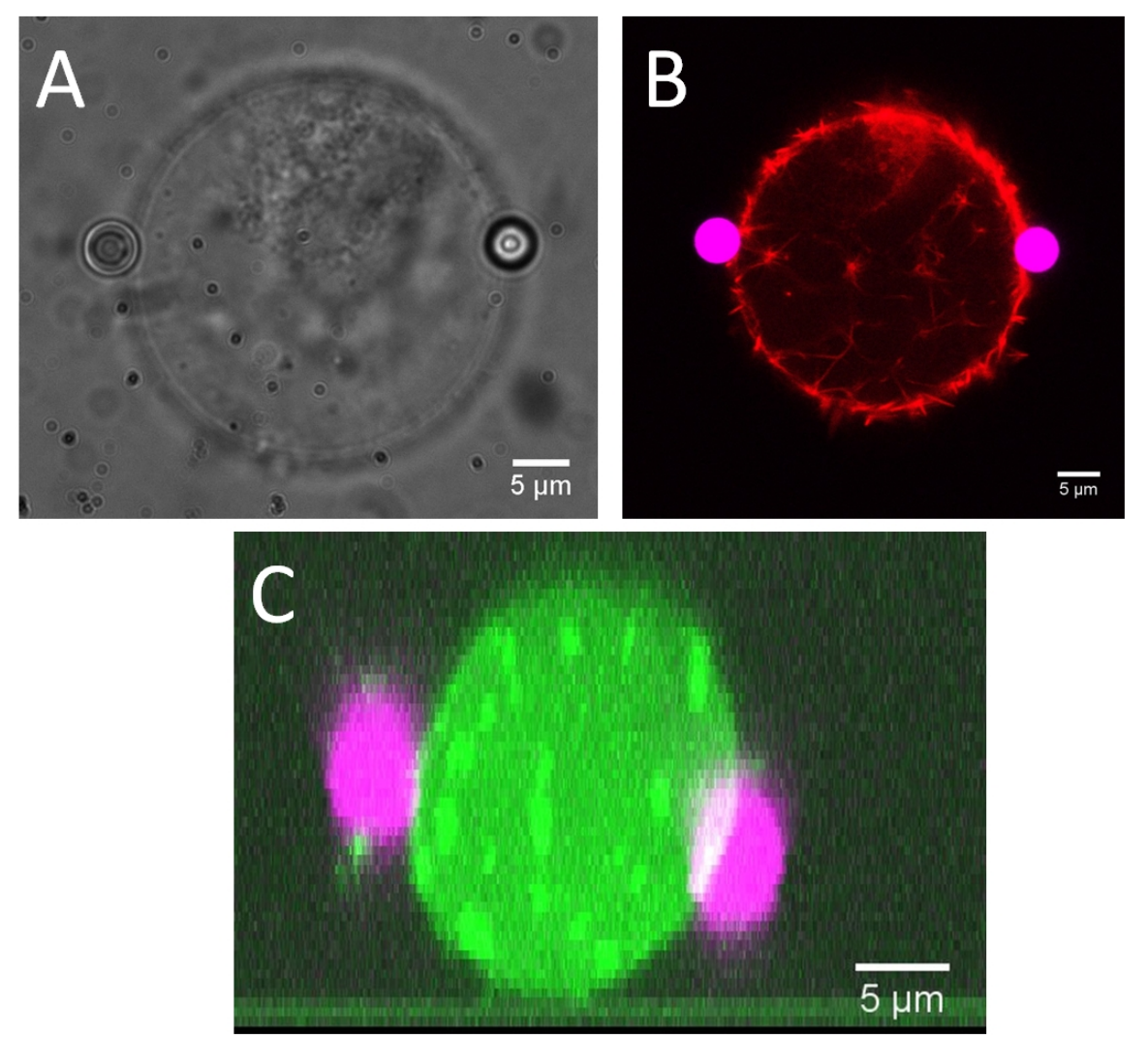

Figure 4.12: Brightfield and fluorescent image of fibroblast. A) Brightfield image of a fibroblast cell suspended between two beads in the optical trap. B) confocal fluorescent image of the same cell. LifeAact-RFP transfected cell to visualize actin (red) and fluorescent beads (magenta). C) $\mathrm{X}-\mathrm{z}$ maximum intensity projection of the same cell. Transfected with non-muscle myosin II-GFP (green). The cell is not trapped but sitting on the coverslip.

force fluctuations of two beads attached to the cell with the optical trap for roughly one hour. Figure 4.14 shows the force curves of two beads attached to a LifeAct transfected fibroblast cell. Figure 4.15 shows stills from a movie recorded of the cell during the force measurements. At the beginning, the actin cortex of the cell is clearly visible with few filopodia sticking out. Until minute 4:30, force generation of the cell is rising to roughly $10 \mathrm{pN}$. In the corresponding image we see that on the right side the cell is starting to wrap around the bead (white arrow) and almost no filopodia are visible anymore. A protrusion is sticking out of the cell. At minute 14:30 the force generation of the cell rises to the maximum level of this measurement of roughly $30 \mathrm{pN}$. There are no clear changes visible in the actin distribution. Between min 14:30 and 29, force generation of the cell is slowly falling to $20 \mathrm{pN}$. The cell-bead system has rotated so that the protrusion is no longer visible. Actin fibers are now visible on the inside of the cortex (arrows). Until minute 45 the external force has been raised and the cell keeps relaxing to the external force. The cortex is getting more roughly and actin fibers on the inside of the cortex are visible. At the end of the recording after 1 hour, the cell force is still around $20 \mathrm{pN}$. No clear changes in the actin distribution are visible compared to the previous image. This measurement shows that it is hard to find clear indications in the actin distribution of a suspended cell to explain changes in the force generation of 20-30 pN. 


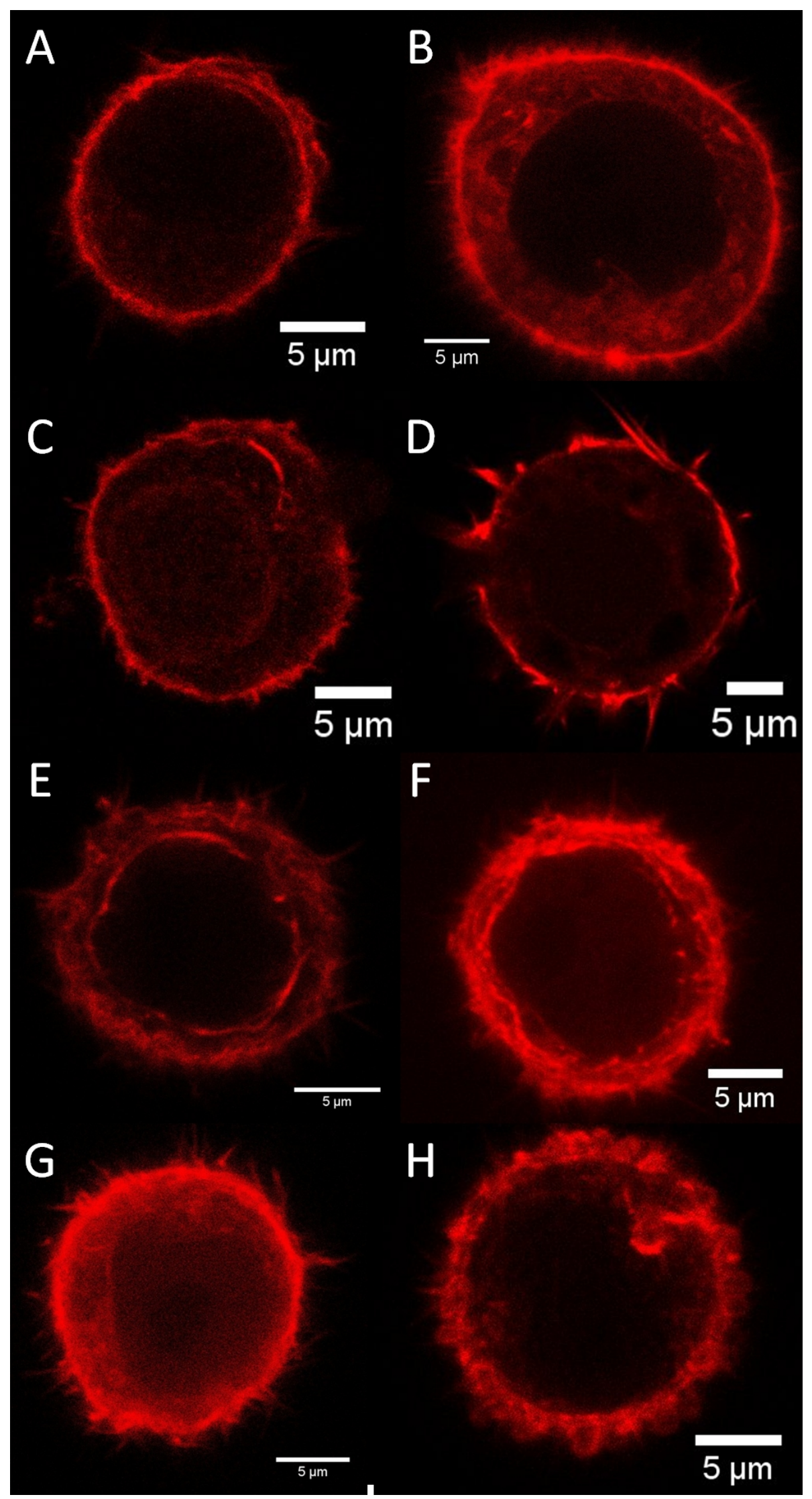

Figure 4.13: Confocal images of NIH 3T3 cells suspended between two trapped beads. This image visualizes the variation of the actin distribution in suspended fibroblast cells. 


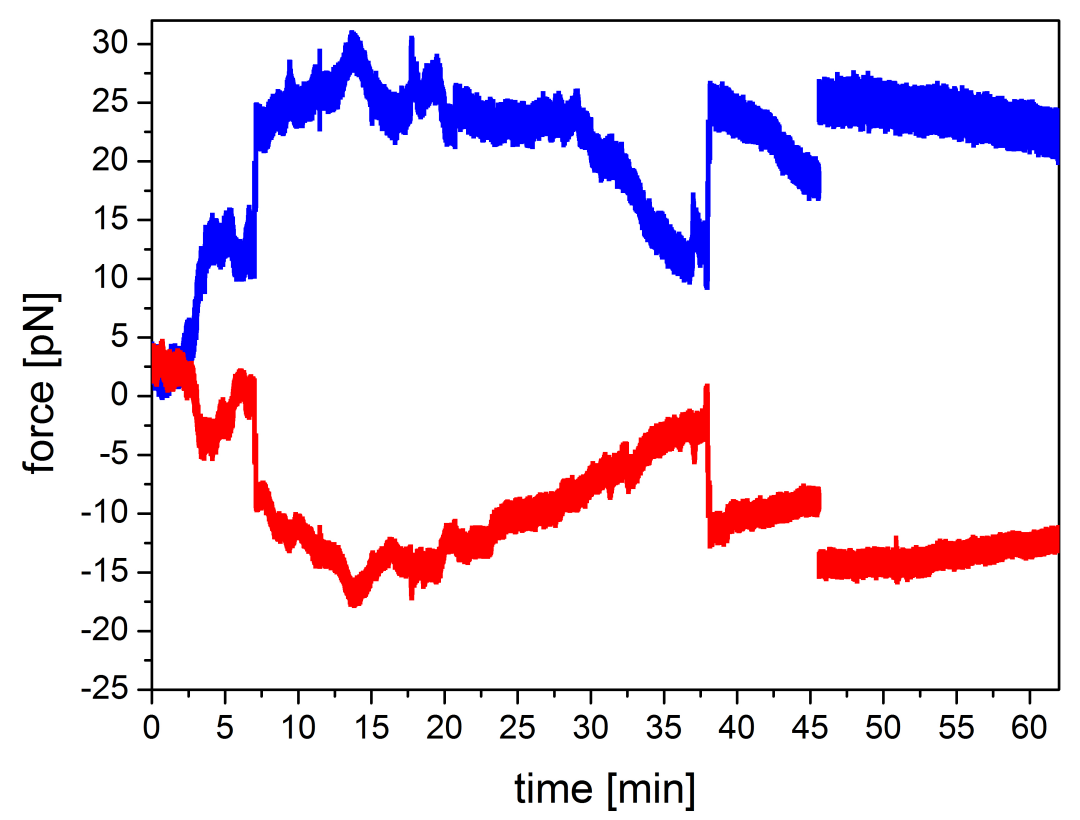

Figure 4.14: Long-time force plots of two beads attached to a fibroblast cell. Force jumps at minute 6,37 and 45 are due to manual increase of the external force by the optical trap.

In the cells presented so far, we have seen that the actin is mostly located in the cortex of the cells. It is interesting to see, if the force production of the cell is influenced by the cortex thickness. Therefore, we do a radial integration of the actin fluorescence image of cells that are suspended between two optically trapped beads and look for changes in cortex thickness. For the radial integration we use the ImageJ plugin "RadialProfileV2" (from Dr. Kota Miura, EMBL Heidelberg). To be able to track changes in the cortex thickness, we compare cardiac fibroblasts in control medium and treated with $100 \mu \mathrm{M}$ blebbistatin as there was a huge, roughly fifty-fold, difference in their force production (see section 5.2.2). Figure 4.16 shows confocal slices of LifeAct transfected cardiac fibroblasts.

For the radial intensity integration, the plugin fits a circle around the threshold image of the cell and integrates the intensity values in a circular fashion, starting from the center. For analysis, the radial intensity integration was normalized for each cell. Figure 4.17 shows a normalized radial intensity distribution averaged over four cells for each condition. We see that the intensity close to the cell center is low and starting to raise with the maximum intensity close to the edge of the cell representing the cortex. We fitted a Gaussian to the peak intensity at the edge of the cell and compare the full width at half maximum values (FWHM) of both fits. The fit is yielding a FWHM of 9.7 for the control cells and 6.1 for blebbistatin cells showing that the actin cortex of control cells is roughly $40 \%$ thicker than the cortex of blebbistatin treated cells. This is just a rough estimate of the cortex thickness as this method is prone to differences in the cortex roughness of cells. A more accurate way to measure the thickness of the cortex independent of its roughness is described in [93], using a co-transfection of actin and membrane dyes. Nevertheless, our results give a hint that the cortex thickness plays a role in force generation of 


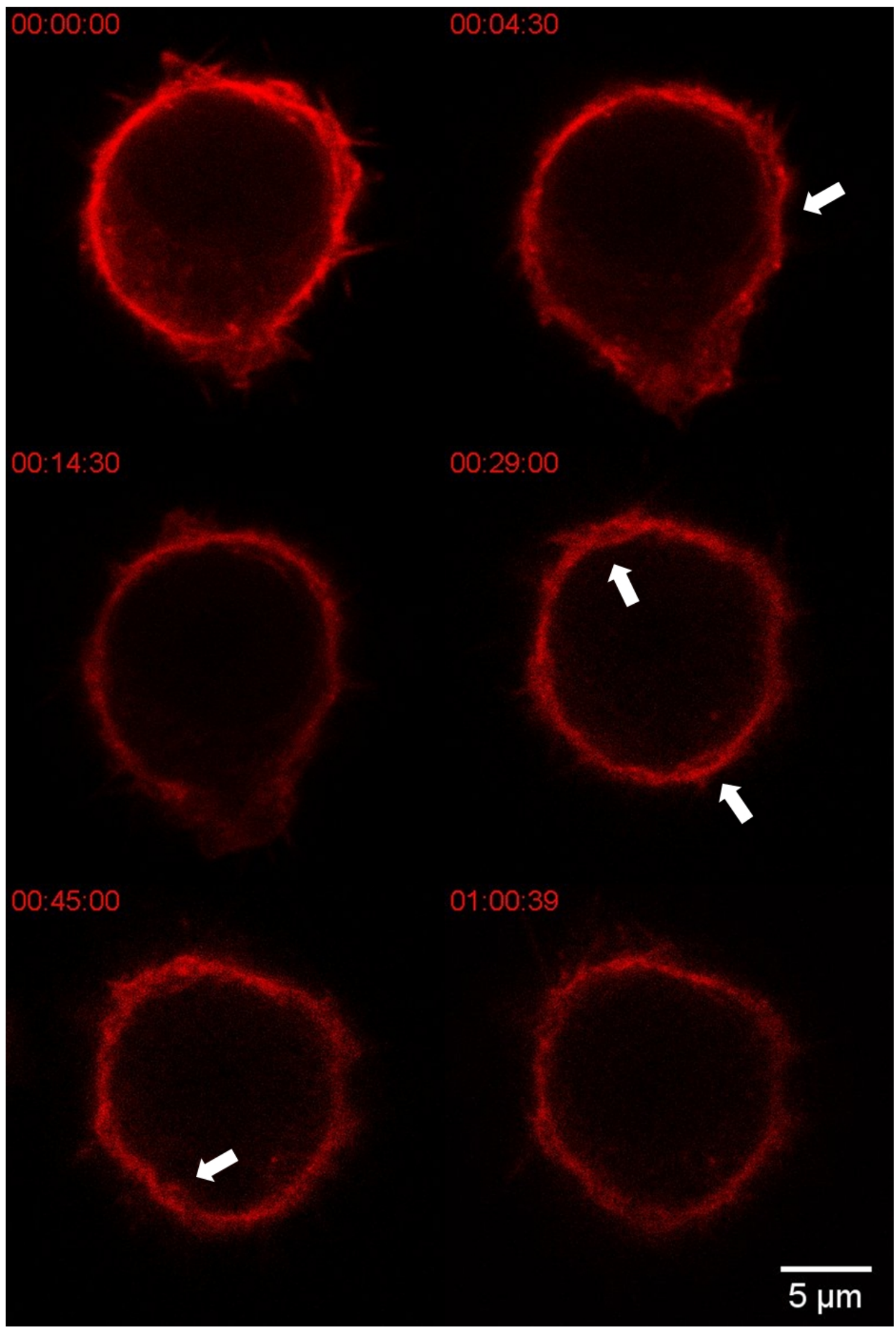

Figure 4.15: Stills from a timelapse recording of a LifeAct transfected fibroblast suspended between two optically trapped beads. Time in hh:mm:ss. Arrow at 4:30 shows how the cell wraps around the bead. Other arrows show new actin fibers on the inside of the cortex. 


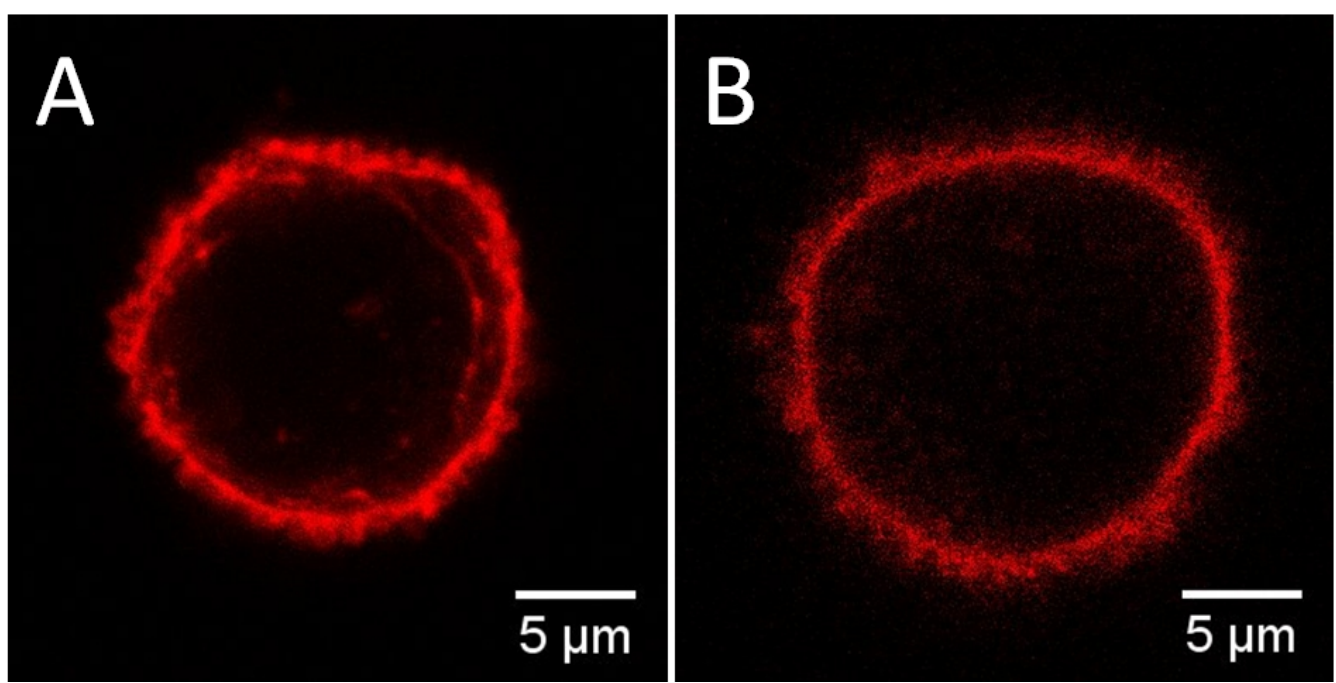

Figure 4.16: Confocal images of LifeAct transfected cardiac fibroblasts (CFB) to visualize the actin distribution. A) CFB in control condition. B) CFB treated with blebbistatin.

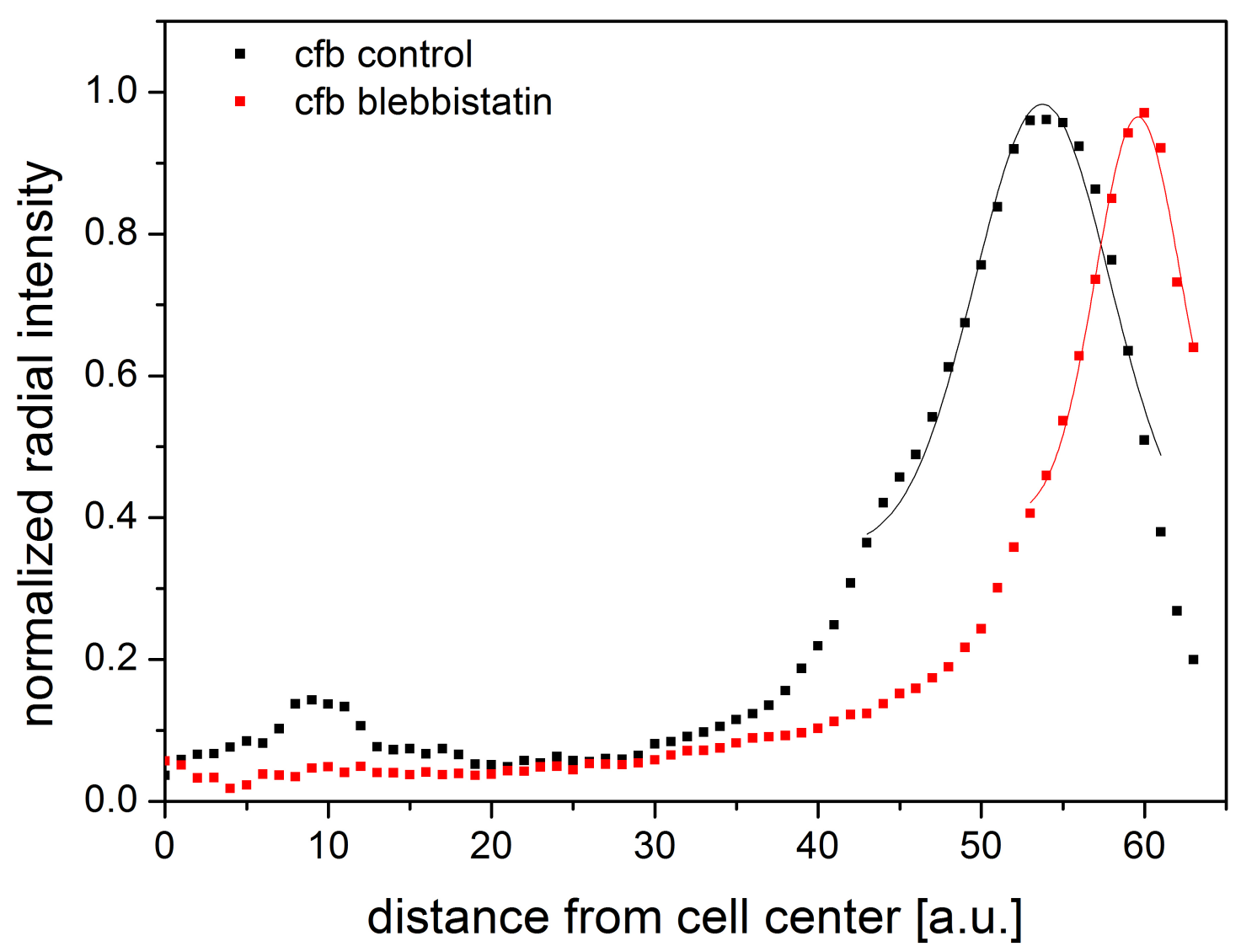

Figure 4.17: Radial intensity distribution of cardiac fibroblasts (CFB) in control medium (black) and treated with blebbistatin (red). The peaks are fitted with a Gaussian fit. Average of four cells for each condition. 
the cells. This result is supported by findings in the just described study of Clark and coworkers that see an increase of the actin cortex thickness by $50-60 \%$ after stabilizing the actin network with jasplakinolide or knock-down of the actin cutting protein cofilin 1.

\section{Structure and distribution of vinculin}

Key elements for transmission of cellular forces to the outside world are, besides the already described acto-myosin network, focal adhesions. Focal adhesions are complexes of proteins which, on the internal side of the cell, link to the force generating elements (acto-myosin) and via transmembrane proteins (e.g. integrins) connect to the extracellular matrix (e.g. fibronectin) [94, 93]. One protein of special interest in this complex is vinculin. It binds to actin on one side and to transmembrane integrins on the other side and therefore making it an interesting object to study due to its force-activated function [95].

To analyze force transmission via focal adhesions of the cell to the beads, we did trapping experiments on a fibroblast cell line with vinculin-venus, a stable fluorescent fusion protein for vinculin, and imaged the cells while trapping. Cells were a gift from Dr. Carsten Grashoff, MPI for Biochemistry, Martinsried, and cultured in the same conditions as the NIH 3T3 cell line described earlier. Figure 4.18 shows confocal images of the vinculin distribution of cells adhering to a glass substrate.
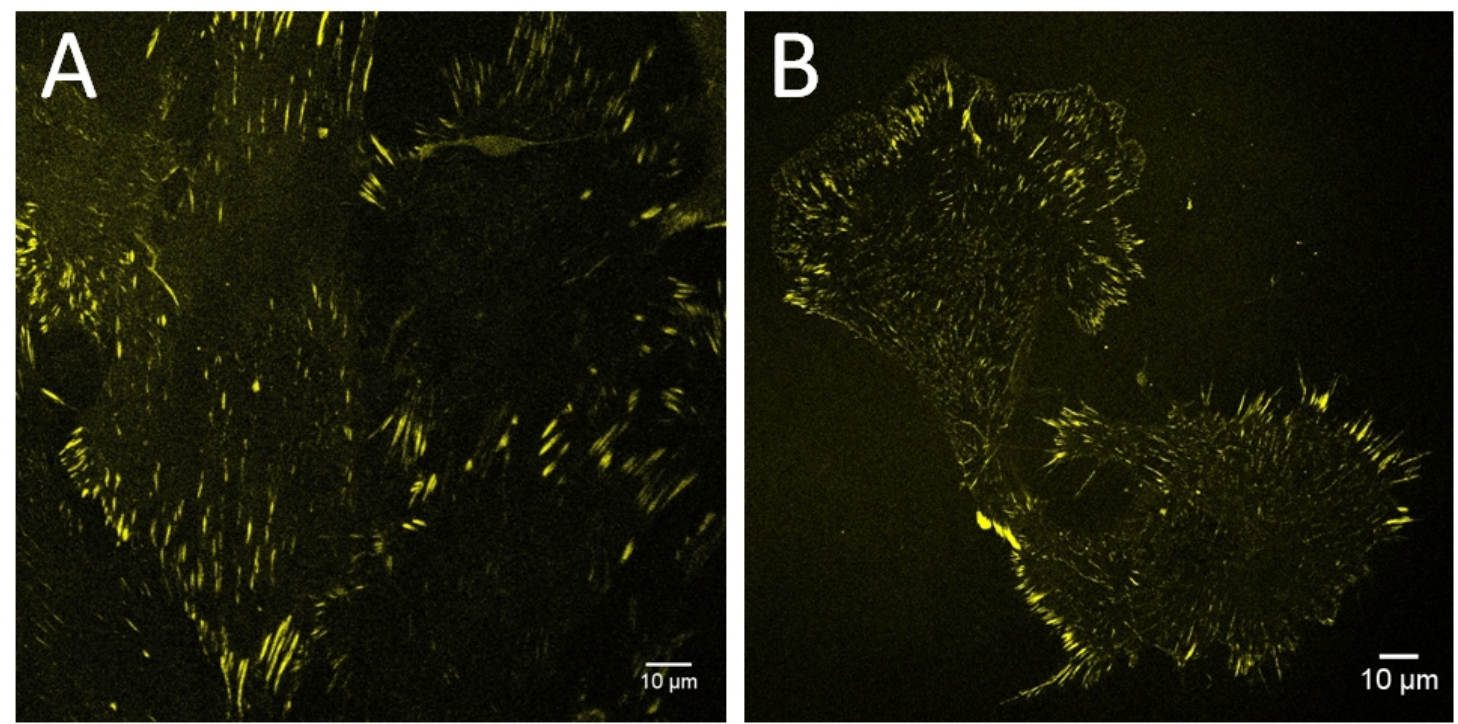

Figure 4.18: Confocal image of fibroblasts sitting on a glass substrate. A) cells are stably expressing vinculin-YFP. B) cells are stably expressing metavinculin-YFP, a spliceosome variant of vinculin.

In a preliminary confocal trapping experiment, we saw an accumulation of vinculin at locations close to the attachment points of the beads (figure 4.19A, white arrows). This suggests formation of a focal complex which connects the acto-myosin 
network to integrins allowing the transmission of forces. However, we also saw accumulations of vinculin at other places with no connection for the cell to the outside.
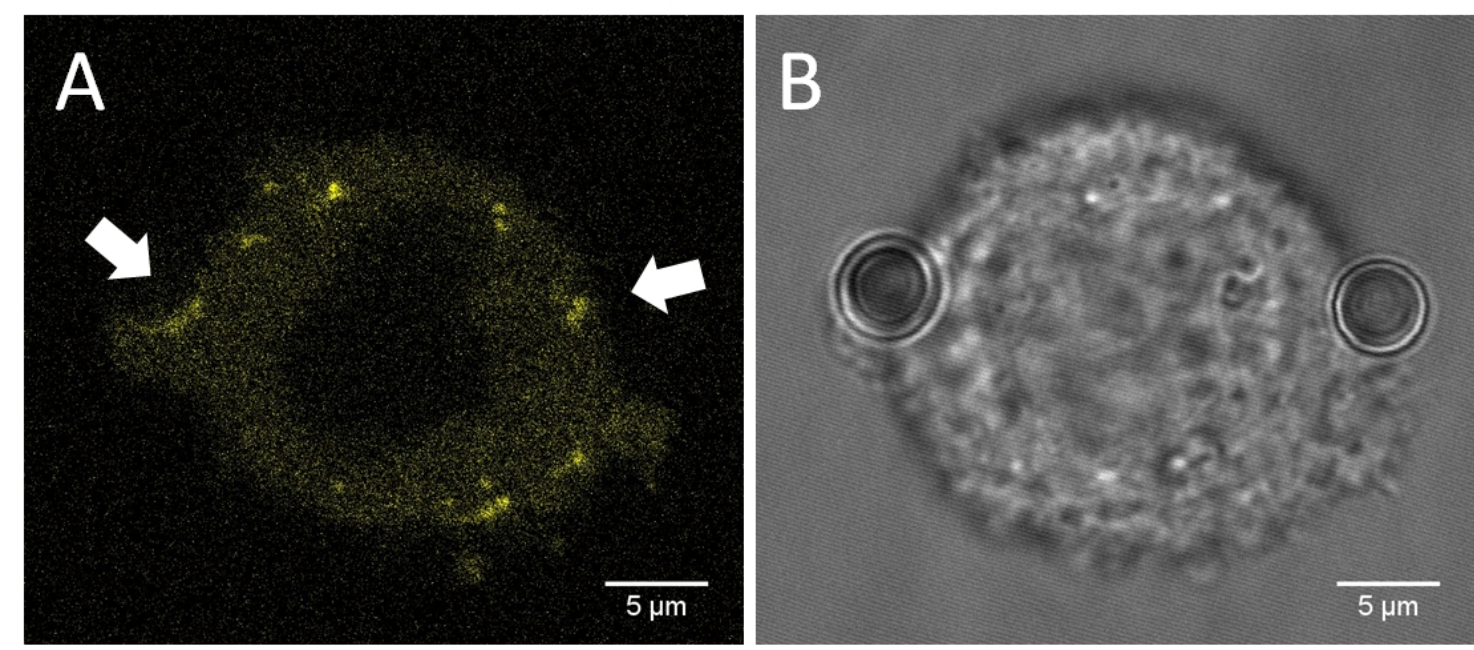

Figure 4.19: Fibroblasts suspended between two optically trapped beads. A) confocal image of vinculin-yfp. B) Brightfield image of the same cell.

\section{$4.4 \quad$ Discussion}

To investigate the distribution of this network and relate them to changes in the force production of the cell we built a novel setup, combining an optical trap with a confocal laser scanning microscope. There has been one description of such a tool [96], but here such an instrument is used for cell measurements for the first time and provided a much faster scanning rate up to $8 \mathrm{kHz}$ which is important for the imaging of contracting cardiomyocytes (see section 5.3). We showed that we can successfully trap beads and combine force fluctuation measurements with confocal imaging of the cell. At the moment, the imaging is limited to the focal plane due to the coupling of the trapping and confocal laser. In the future, we will work to overcome this limitation by using time gated traps [97]. This means that we will shutter the trapping laser during imaging of different z-planes.

Images of the actin distribution in cells showed that there is a highly diverse population of cells, making it hard to couple force generation to changes in the actin structures of the cell. To overcome this problem we will synchronize the cells in their cell cycle, hoping that the distribution of cells would be more even afterward. There is also need for markers in the actin distributions that are available for quantitative analysis to look for changes. One option, as already mentioned is the thickness of the cell cortex, but for a precise measurement, one would need a second label for the plasma membrane [93]. To visualize subtle changes in the actin cortex, further improvement of the resolution is necessary. The setup is being equipped with a time 
gated STED system right allowing for resolutions much below $100 \mathrm{~nm}$ and will help to visualize the acto-myosin network in even greater detail.

Preliminary experiments have showed that we are able to observe an accumulation of the focal adhesion protein vinculin at contact points of the beads. In future experiments, we want to go into more detail, scanning the attachment region with high resolution. We plan to use vinculin proteins with FRET sensors [95] to combine force measurements with recordings of the FRET signal. This will allow us to look for differences in focal adhesion complexes in 2D and 3D environments, a topic that has not been looked into in great detail, yet [98]. 



\section{Mechanical properties of cells in engineered heart muscle}

The human heart is a very outstanding organ of our body. It is the largest muscle, beating over 2 billion times and pumping over 150 million liters of blood during an average lifespan. However, myocardial infarction and heart failure are the main cause of death in industrialized countries and generate enormous costs for our society. Ischemic heart diseases like myocardial infarction ('heart attack') are the number one killer in the western world [99], cardiomyopathies affect up to $2 \%$ of births $[100,101]$ and cardiotoxicity of drugs is a leading cause of their withdrawal from the markets [102]. Therefore, there is an urgent need for cardiocyte models to study cardiac diseases and treatments. The project described in this chapter is part of a collaboration with the group of Prof. Zimmermann in the pharmacology department of the university medical center in Göttingen.

\section{Engineered heart muscle}
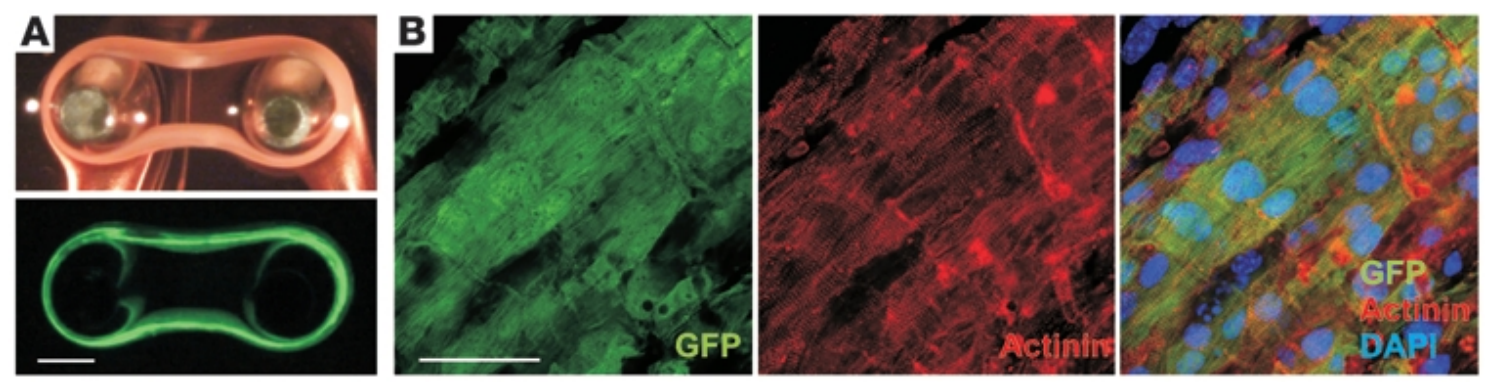

Figure 5.1: Engineered heart muscle. A) Light microscope (top panel) and epifluorescence images (bottom panel) of an EHM. B) Confocal laser scanning microscopy of a cardiomyocyte bundle in EHM. EGFP (green), $\alpha$-actinin (red), nuclei (blue, DAPI). Scale bars A) $1 \mathrm{~mm}, \mathrm{~B}) 50$ $\mu \mathrm{m}$. Adapted from [103]

One approach to generate heart models is tissue engineering. It should provide biological substitutes to experimentally test in vitro and in vivo applications including drug development, disease modeling and tissue reconstruction for therapeutic issues. Tissue engineering relies on the properties of isolated cells to organize themselves into tissue equivalents under appropriate in vitro conditions in laboratory incubators or bioreactors. Most of the approaches to generate heart tissue under these conditions involve adding extracellular matrix components to the cell culture 

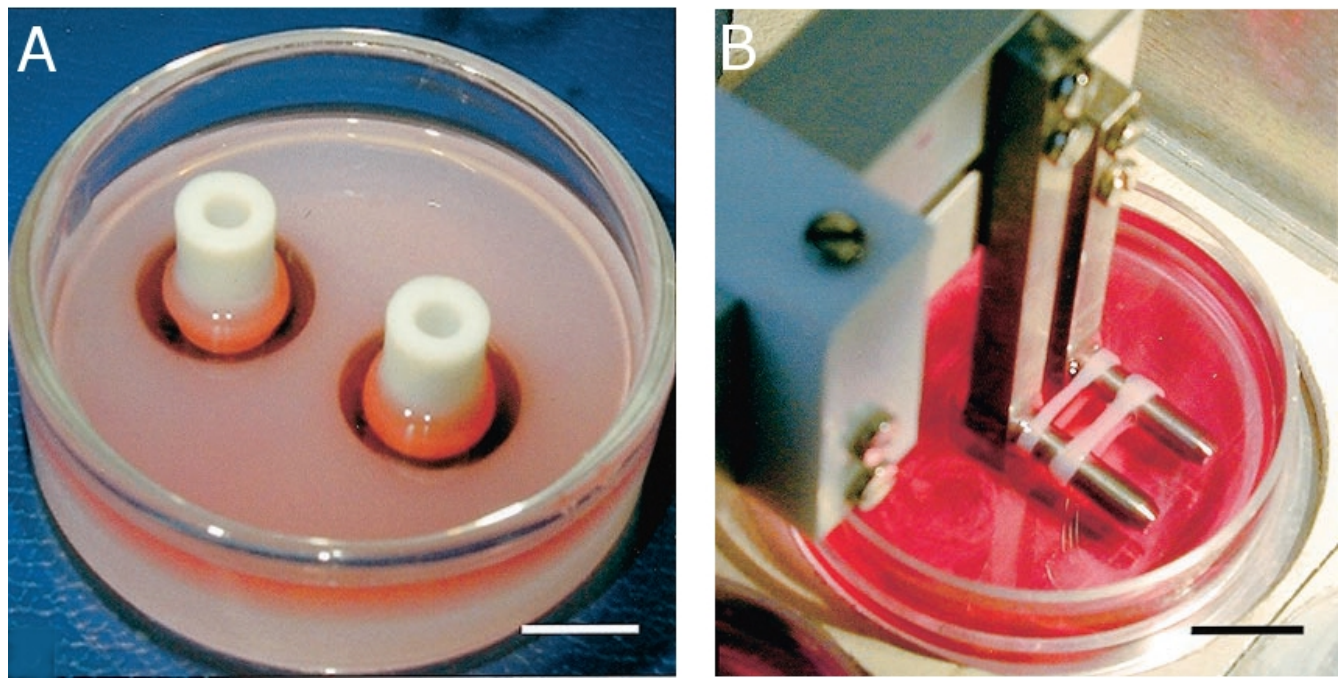

Figure 5.2: Experimental setup for EHT preparation. A: Condensation of EHM rings around teflon cylinders in casting molds. B: EHT in a stretch apparatus to culture the tissue under cyclic stretch. Scalebars $=10 \mathrm{~mm}$. Adopted from [107]

which acts as scaffold. One of the first methods to obtain such myocardial tissue in a systematically way was obtained by Zimmermann and coworkers roughly 15 years ago [104, 105]. They modified a technique that was used to measure contractile forces of fibroblast cells spanning them between two separated collagen-coated rods in culture medium [106]. Instead of fibroblasts, they seeded the collagen hydrogel with neonatal rat cardiac myocytes to generate engineered heart tissue (EHT), later called engineered heart muscle (EHM) (figure 5.1). Figure 5.2 shows images of how the EHM are produced. These EHT fulfill three fundamental properties that are necessary for use as a cardiocyte model: They are excitable, they have anisotropic structure and function and they can generate considerable forces up to the millinewton regime [107]. To provide a better transfer of findings made with EHM to humans, people successfully generated EHM with human cells. However, EHM made from myoblasts, bone marrow- or cardiac stem cell have certain limitations in EHM production. Their upscaling in ex vivo conditions to clinically relevant numbers is challenging, the cardiomyocyte yield is low or they are not able to differentiate into cardiomyocytes and establish electromechanical junctions with host cardiomyocytes [108]. One option to overcome this limitations is the use of pluripotent stem cells (PSCs). But ethical reservations make it hard to use embryonic PSCs. A solution to overcome this problems has been developed in the last years. It became possible to reprogram other cell types e.g. fibroblasts to regain their pluripotency [109, 110]. These human induced pluripotent stem cells (hiPSCs) are the cells of choice to generate EHM from human derived cells. Figure 5.3, shows the process to reprogram fibroblasts or mesenchymal stem cells (MSCs) into induced pluripotent stem cells. Fibroblast cells are infected with a lentiviral system which contains four reprogramming factors [112]. After infection, cells are mechanically expanded for three to four passages and generated hiPSCs are cultured on mouse embryonic fibroblasts in human embryonic stem cell medium. After completion of the reprogramming process, cells can be differentiated into cardiomyocytes. For differentiation, hiPSCs are dispersed into clumps with collagenase IV and cultured in suspension first in human ESC medium and later in differentiation medium. Cells are then plated on 


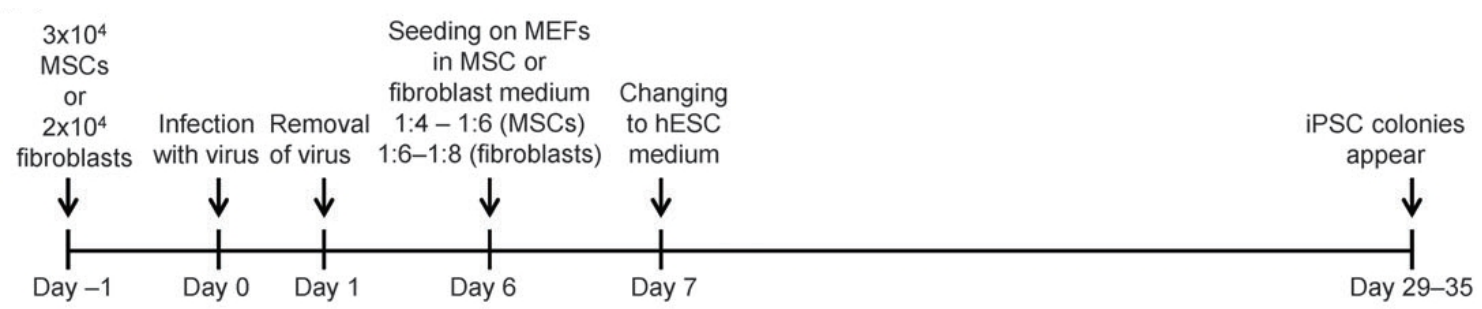

Figure 5.3: Generation and characteristics of human induced pluripotent stem cells derived from mesenchymal stem cells and fibroblasts. Cells are infected with a lentivirus containing reprogramming factors. After the infection process is completed, they are cultured on mouse embryonic fibroblasts (MEFs) in human embryonic stem cell medium. Adapted from [111]

gelatin-coated dishes and further cultured in differentiation medium for 30 days. After the differentiation process, beating clusters of cardiomyocytes are picked and used to generate EHM. A detailed protocol of the reprogramming and differentiation process can be found in [111]. Figure 5.4 shows a schematic of the general process to differentiate stem cells into cardiomyocytes.

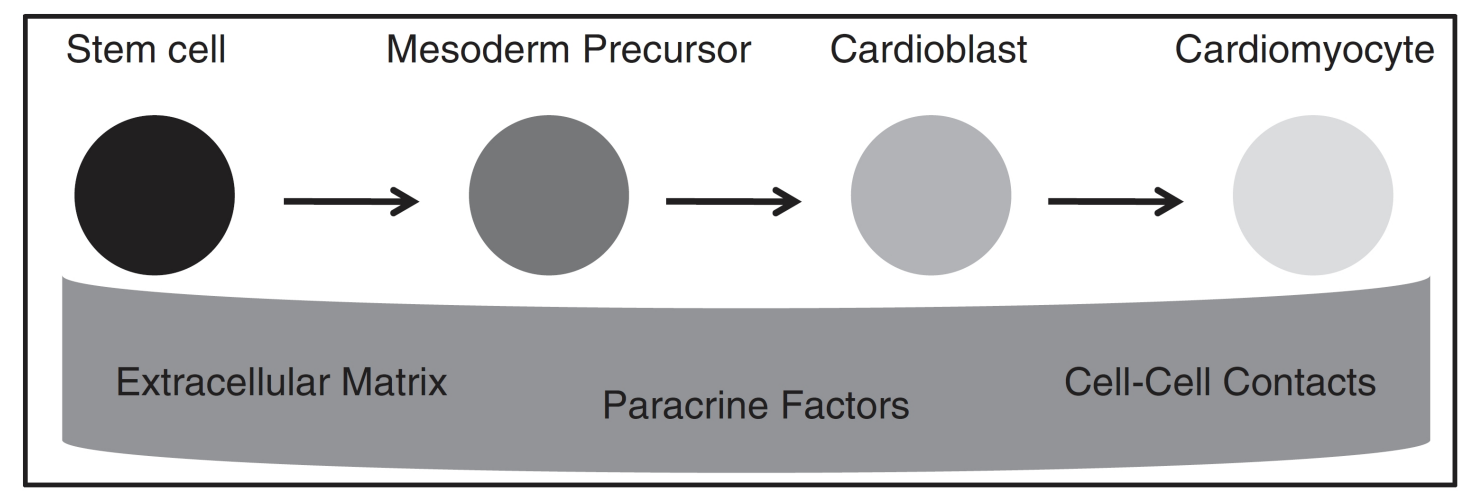

Figure 5.4: Schematic of the general process to differentiate stem cells into cardiomyocytes. Undifferentiated stem cells need distinct paracrine, stromal and cellular stimuli to differentiate into cardiomyocytes. Adapted from [113]

\section{Non-myocyte cells in EHM}

A crucial factor in the development of EHM is the presence of non-myocyte cells (fibroblasts, smooth-muscle cells and endothelial cells). This is not surprising as the human heart tissue is composed only $20 \%-30 \%$ of myocytes and $70 \%$ of non-myocytes [114]. Non-myocytes play a crucial role in heart- and cardiac myocyte development, function and support the growth of myocytes in culture. They have high impact on the function of engineered constructs $[115,116]$. EHMs made from an unpurified native heart mix develop threefold higher forces than those made from purified cardiac myocytes [117]. Furthermore, pure, genetically selected cardiac myocytes from human PSCs did not form engineered 3D tissue until the addition of fibroblast cells [118], and addition of endothelial and stromal cells to hESC derived cardiac myocytes improved cardiac tissue structure and function [119, 120]. Interestingly, the high content of $50-80 \%$ of cardiomyocytes in the starting conditions of the EHM 
reconstitution mixture drops to the natural content of living hearts of $\approx 30 \%$ within only 3 days due to apoptosis [121], whereas fibroblasts were unaffected [122].

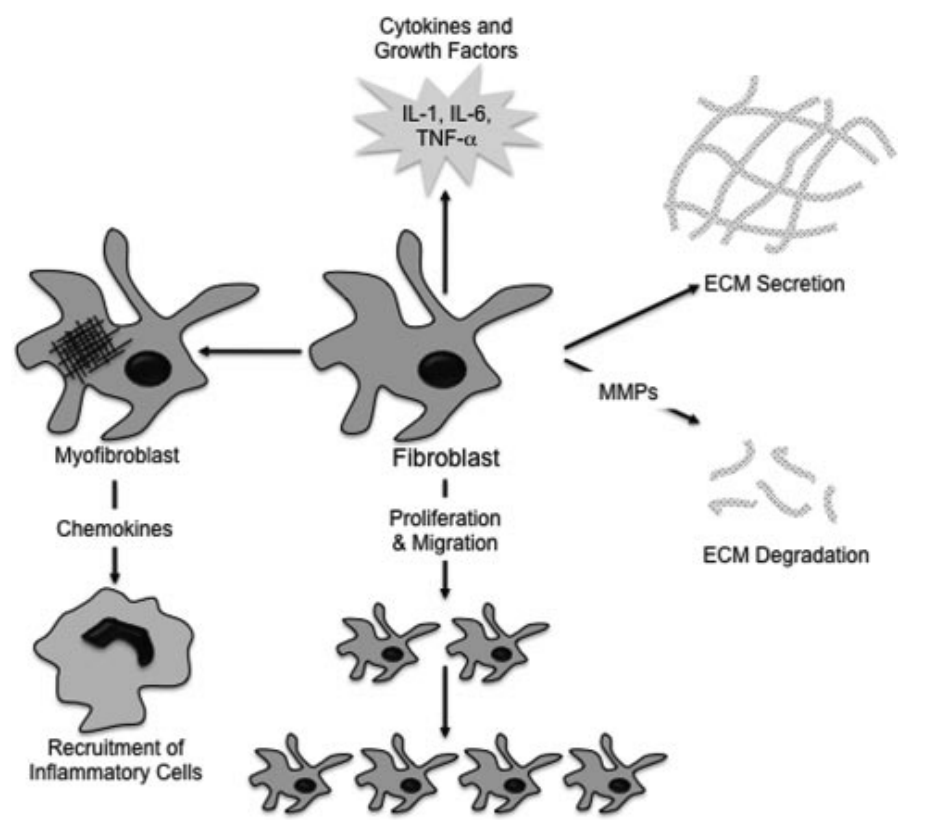

Figure 5.5: Overview over the roles of cardiac fibroblasts. It generates and alters the extracellular matrix, secretes different growth factors, provides proliferation and can differentiate into myofibroblasts. Adapted from [123]

There are many ways how cardiac fibroblasts could influence the development and function of heart tissue. A general overview of the functions is shown in figure 5.5. Fibroblasts may communicate with cardiomyocytes already at early stages, assisting them during development. They are located within the developing cardiac muscle and proliferate. They express various components of the extracellular matrix (ECM) including fibronectin, collagens, and hyaluronan and proteoglycan link proteins. Interestingly it was shown that embryonic cardiomyocytes grown on platelets with a composition of those matrix proteins showed an increase in proliferation whereas embryonic mouse cardiomyocytes showed rather a hyperthrophic than a proliferative phenotype when cultured together with adult cardiac fibroblasts [124] (figure 5.6). This suggests that cardiac fibroblasts take also part in steering of the development of cardiomyocytes.

Cardiac fibroblasts are also constantly subject to mechanical stress during cardiac function. It has been shown that mechanical stimuli play a critical role in the function of these fibroblasts. They can upregulate the expression of ECM components, ECM receptors and different cytokines and growth factors [126, 127, 128].

\section{\begin{tabular}{l|l} 
Force fluctuations and elasticity of primary \\
fibroblasts
\end{tabular}}

Nonmyocyte cells like fibroblasts are crucial for the development of hiPSC into functional bioartificial cardiac tissue [118]. It was found that engineered heart tissues that were developed with different primary fibroblasts have different rheological behavior. Experiments using a rheometer showed that EHM with cardiac fibroblasts are stiffer that EHM with skin fibroblasts (personal communication 2015, Susanne 

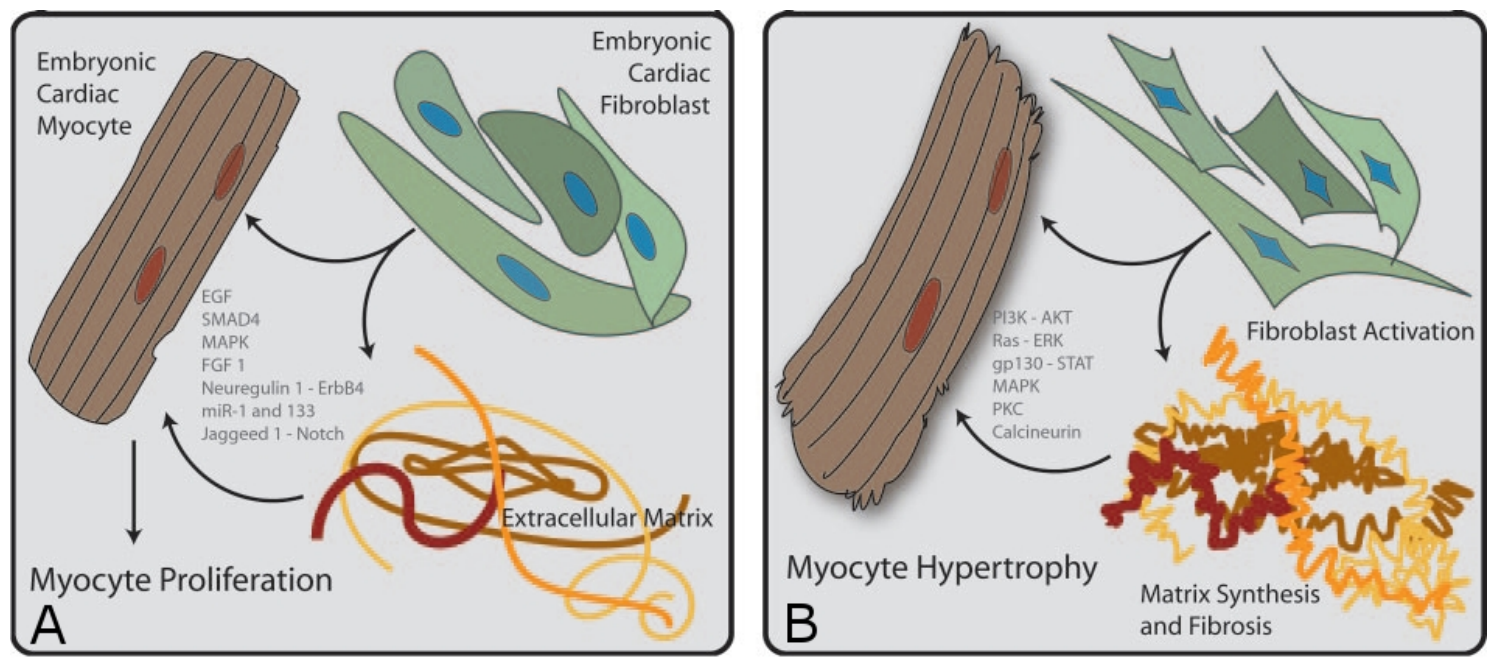

Figure 5.6: Interactions of cardiac fibroblasts with their environment at different developmental stages. A) In the developing heart, fibroblasts express extracellular matrix (ECM) proteins and growth factors that promote cardiomyocyte proliferation. B) In adult hearts, under mechanical stress, cardiomyocytes grow in a hyperthrophic manner stimulated by signals from the ECM and adult cardiac fibroblasts. Adapted from [125].)

Schlick, Pharmacology, UMG). As mechanical stimuli play an important role in the function of cardiac fibroblasts, it is interesting to investigate if fibroblasts from other parts of the body or ihPSC-derived have other mechanical properties than their native counterpart from the heart, cardiac fibroblasts. Therefore we use the optical trap setup, to measure mechanical properties of different primary fibroblast cells. We measured the active and passive mechanical properties of cardiac fibroblasts (CFB), human foreskin fibroblasts (HFF), gingival fibroblasts (GFF) and two cells that were hiPSC-derived, NM WT cells and NM 257 cells (figure 5.7). All cells are primary human cells and were used according to the guidelines of the university medical center Göttingen. Due to the limited quantity of the primary cells, the sample size of some cell types is rather small. Therefore we can only make careful statements for these cell types.

\section{Elasticity}

We obtain the effective spring constant from force-elongation plots of each individual cell as described in sections 2.4 and 3.2. To elucidate, if fibroblasts from different parts of the body have different elastic properties, we performed active deformation experiments on all different primary cell types. Figure 5.8 and table 5.1 show the results of the experiments. We find no significant difference for the spring constants of all cells. However, non-muscle myosin II inhibition of CFB with blebbistatin reduced the spring constant significantly to half of that of the untreated ones ( $\mathrm{p}>95 \%)$. Although the reduction is not as strong compared to the NIH 3T3 fibroblasts, this shows that also in primary fibroblasts the acto-myosin network plays a large role in the rigidity of the cells. 

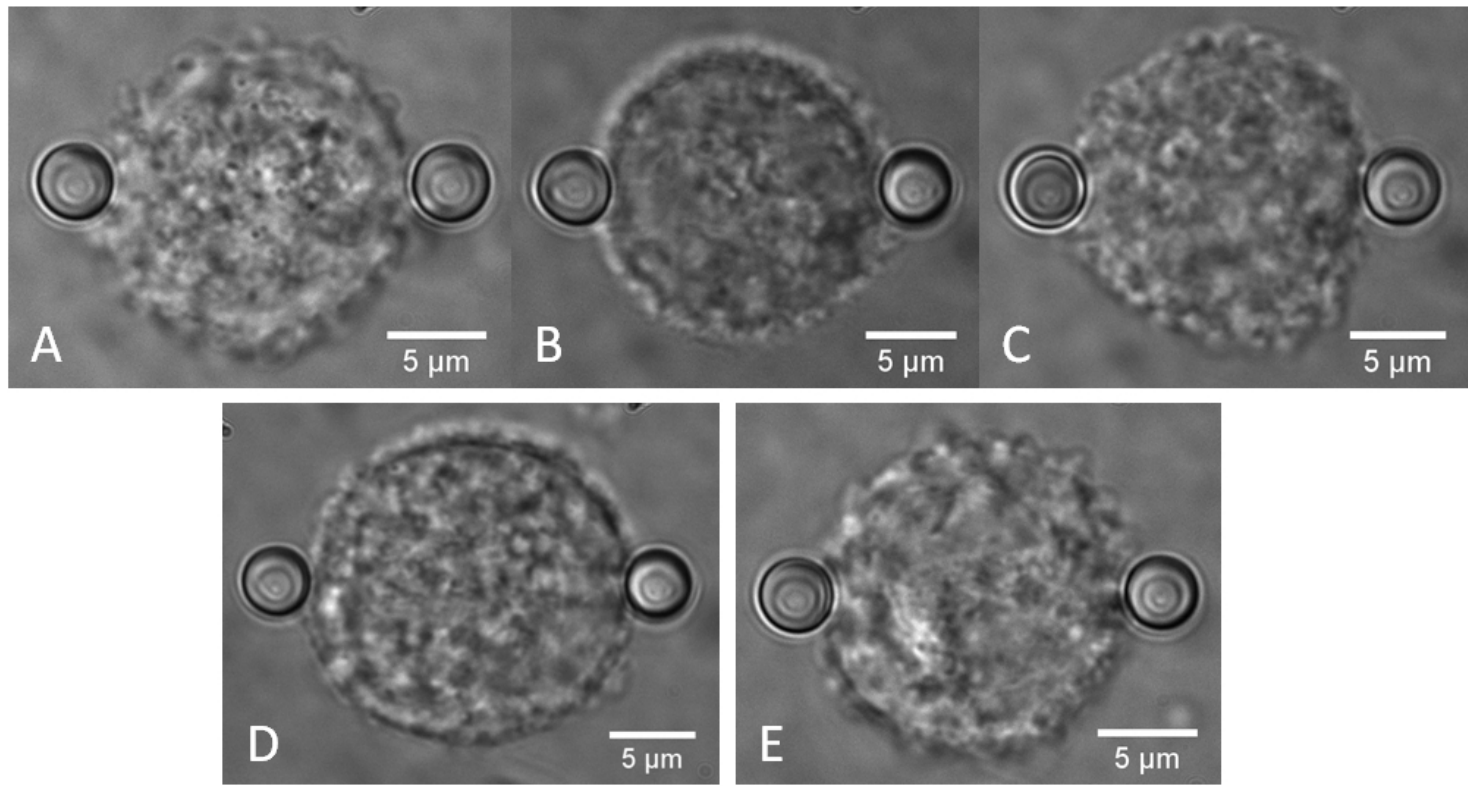

Figure 5.7: Microscope images of primary fibroblasts suspended between two optically trapped beads. A) cardiac fibroblast, B) human foreskin fibroblast, C) gingival fibroblast, D) and E) hiPSC-derived fibroblast cells NM 257 and NM WT.

\begin{tabular}{|c|c|c|}
\hline cell type & $k_{\text {cell }} \times 10^{-5} \mathrm{Nm}^{-1}$ & sample size $(\mathrm{N})$ \\
\hline \hline CFB & $4.63 \pm 3.07$ & 17 \\
\hline $\mathrm{CFB}+100 \mu \mathrm{M}$ blebbistatin & $2.04 \pm 1.08$ & 10 \\
\hline $\mathrm{HFF}$ & $4.60 \pm 1.63$ & 11 \\
\hline $\mathrm{GFB}$ & $2.64 \pm 1.26$ & 3 \\
\hline $\mathrm{NM} 257$ & $4.04 \pm 1.73$ & 5 \\
\hline $\mathrm{NM} \mathrm{WT}$ & $3.02 \pm 0.78$ & 8 \\
\hline NIH 3T3 & $9.51 \pm 2.56$ & 10 \\
\hline
\end{tabular}

Table 5.1: Effective spring constants for human primary cells. Cardiac fibroblasts (CFB), human foreskin fibroblasts (HFF), gingival fibroblasts (GFB) and hiPSC derived fibroblasts (NM WT and NM257)

The mean spring constant of gingival fibroblasts is only roughly half of that of the CFB but this difference is not statistically significant at a level of $95 \%$. This is probably mainly due to the low N-value of GFB cells of only 3 measurements. More measurements of this cell type need to be done in the future to see if the cells are really softer than CFB and HFF cells. hiPSC-derived fibroblasts have an effective spring constant that is comparable to the primary cells, showing that the differentiation of hiPSCs leads to same results as the development in vivo. Compared to the cell line NIH 3T3, all primary cells are significantly ( $\mathrm{p}>95 \%$ ) roughly two times softer. It is not clear if the difference is due to the different origin of the cells (human and mouse) or if this is due to the fact that cells of the cell line are immortal. 


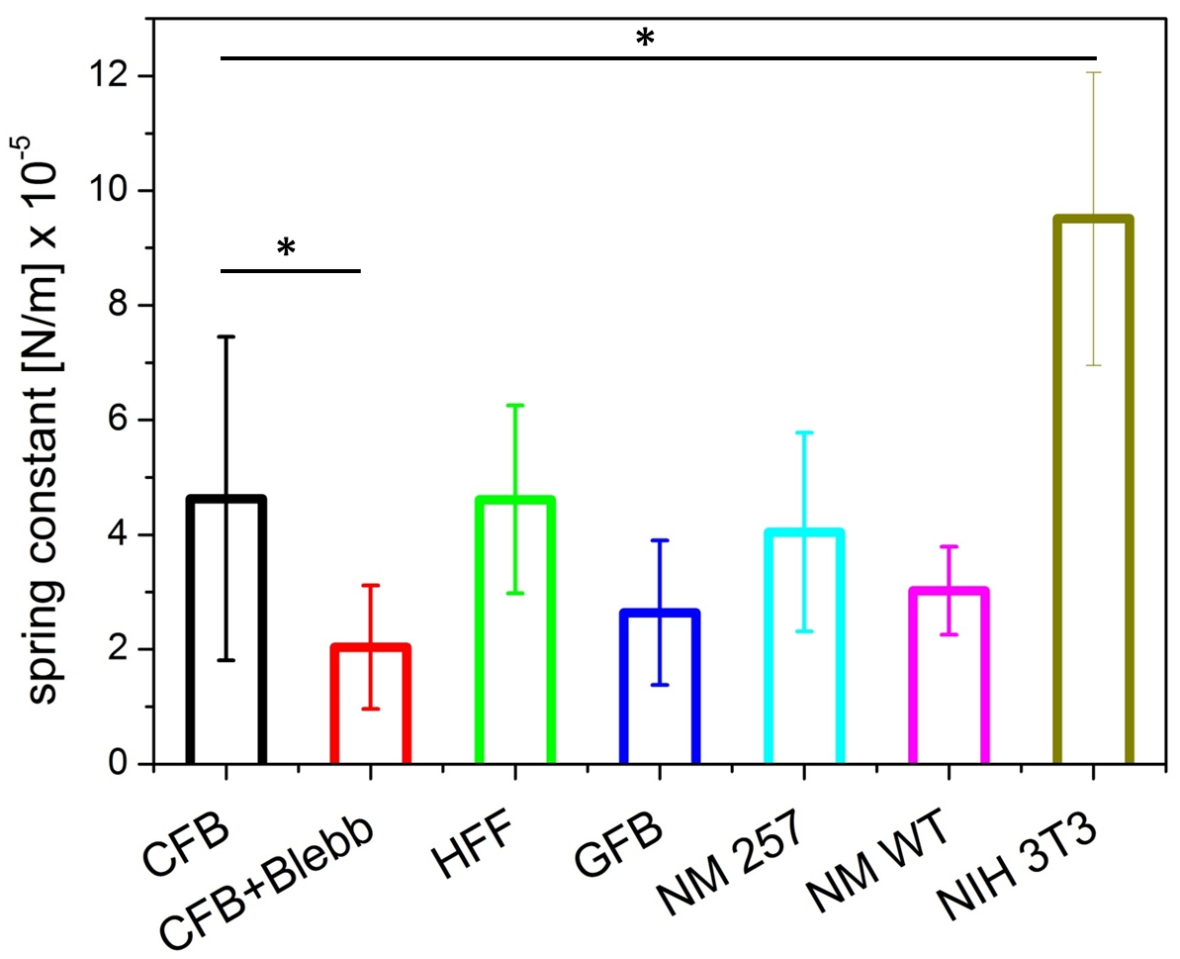

Figure 5.8: Effective spring constants of primary fibroblasts. Cardiac fibroblasts in control conditions (CFF, black) and after treatment with $100 \mu \mathrm{M}$ blebbistatin (CFB+Blebb, red). Human foreskin fibroblasts (HFF, green), gingival fibroblasts (GFB, blue), hiPSC-derived fibroblasts (NM257 and NM WT, light blue and pink), and NIH 3T3 FB (dark yellow).

\section{Contractile force fluctuations}

To quantitatively analyze the force fluctuations that the primary fibroblasts generate, we used the passive mode of our trapping setup to detect the low frequency position fluctuations of the beads that are attached to the suspended cells. We follow the analysis described in sections 2.3 and 3.3 to get a quantitative estimate of the total force production $\sum\left\langle F F^{*}\right\rangle$. Figure 5.9 shows mean values of integrated cellular force fluctuations and table 5.2 presents the corresponding numbers. For cardiac fibroblasts, we find that treatment with blebbistatin significaltly $(\mathrm{p}>95 \%)$ reduced the fluctuations more than fifty-fold. This shows the huge influence of the acto-myosin network to the stress fluctuations. Force fluctuations of human foreskin fibroblasts are also significantly reduced by roughly one order of magnitude compared to CFB, although the scatter in both samples is quite high. Due to the limited sample size of the other primary cells, statistical analysis was not possible. Although the mean value of force fluctuations of GFB and NM WT were on the same level as the HFF, they were not statistically significantly different from CFB. Interestingly the mean value of force fluctuations of NM 257 is on the same level as the cardiac fibroblasts but due to a large scatter in the dataset and low sample size no statistical significant conclusions can be drawn. 


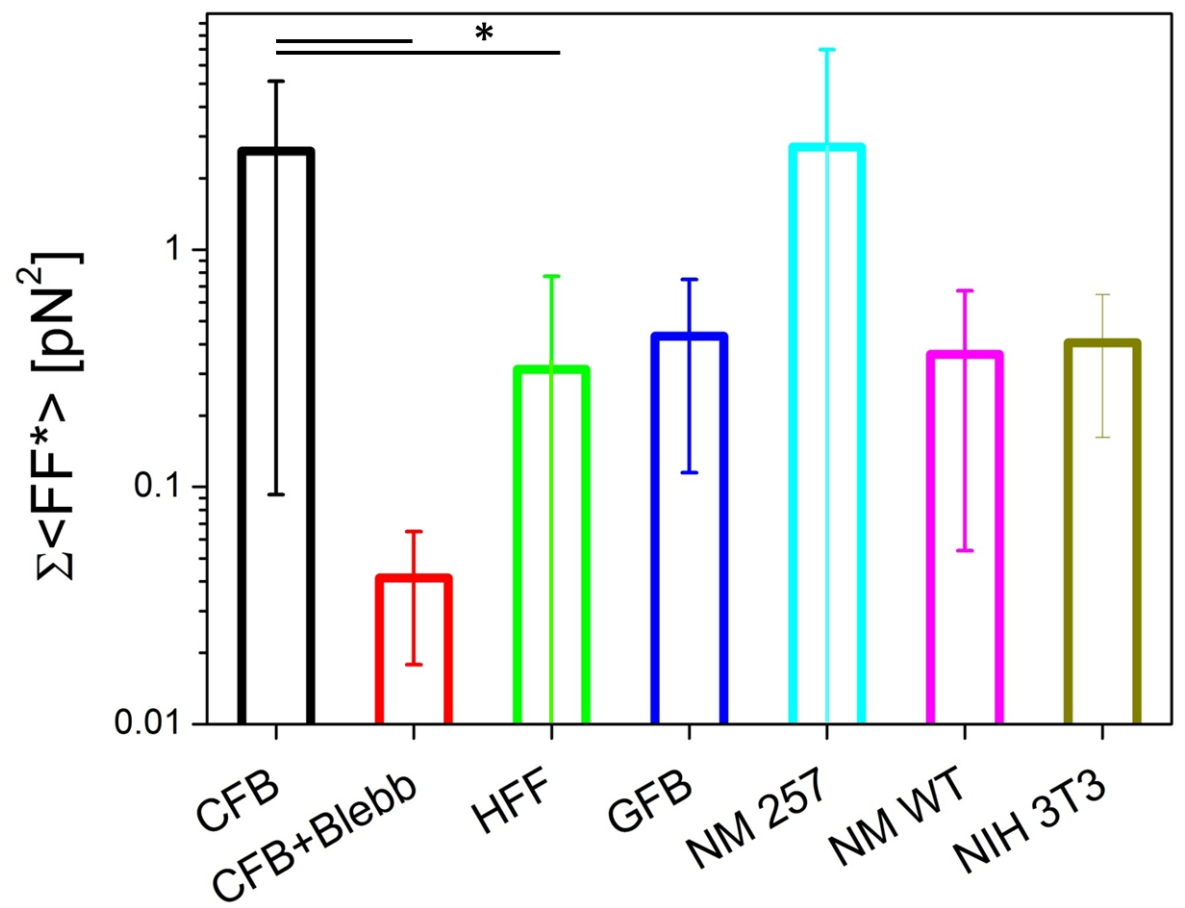

Figure 5.9: Integrated cellular force fluctuations $\sum\left\langle F F^{*}\right\rangle$ of human primary cells. Cardiac fibroblasts in control conditions (CFF, black) and after treatment with $100 \mu \mathrm{M}$ blebbistatin (CFB+Blebb, red). Human foreskin fibroblasts (HFF, green), gingival fibroblasts (GFB, blue), hiPSC-derived fibroblasts (NM257 and NM WT, light blue and pink), and NIH 3T3 FB (dark yellow).

\begin{tabular}{|c|c|c|}
\hline cell type & $\sum\left\langle F F^{*}\right\rangle \mathrm{pN}^{2}$ & sample size $(\mathrm{N})$ \\
\hline \hline CFB & $2.61 \pm 2.52$ & 17 \\
\hline CFB+100 $\mu$ M blebbistatin & $0.041 \pm 0.024$ & 10 \\
\hline HFF & $0.31 \pm 0.46$ & 11 \\
\hline GFB & $0.43 \pm 0.32$ & 2 \\
\hline NM257 & $2.71 \pm 4.26$ & 5 \\
\hline NM WT & $0.36 \pm 0.31$ & 4 \\
\hline NIH 3T3 & $0.41 \pm 0.24$ & 30 \\
\hline
\end{tabular}

Table 5.2: Integrated cellular force fluctuations $\sum\left\langle F F^{*}\right\rangle$ of human primary cells. Cardiac fibroblasts (CFB), human foreskin fibroblasts (HFF), gingival fibroblasts (GFB) and hiPSC derived fibroblasts (NM 257 and NM WT) 


\section{Cardiomyocytes}

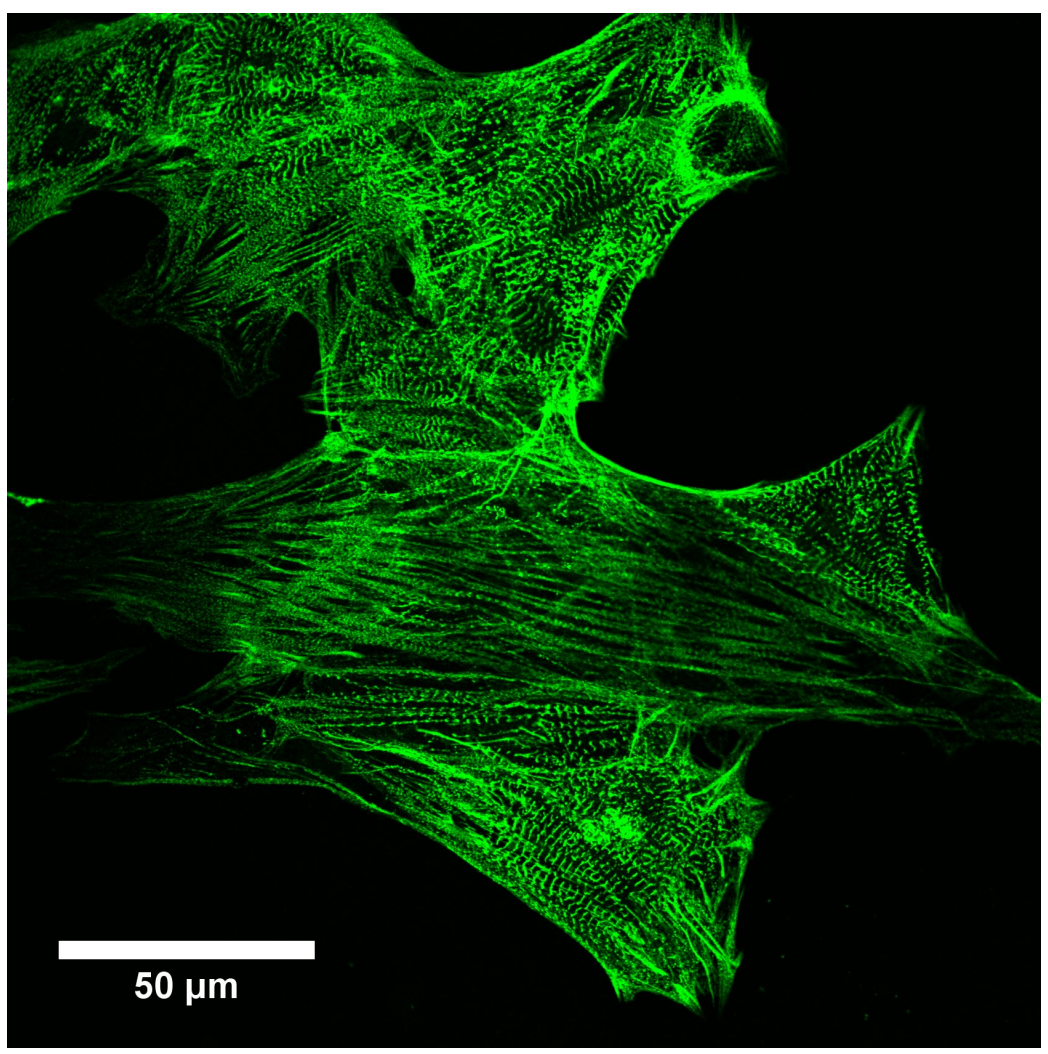

Figure 5.10: Confocal image of human induced pluripotent stem cell derived cardiomyocytes. Staining for $\alpha$ actinin. Image taken by Til Driehorst.

Cardiomyocytes are relevant for the generation of high contractile forces and relevant for the collective beating motion of the whole tissue. For this purpose, they developed sarcomeres, strong acto-myosin bundles that can exert high contractile forces (figure 5.10). It is interesting to compare force generation of cardiomyocytes of different origins (rat, mouse, human, iPSC-derived) that are used for EHT. The force generation of cardiomyocytes at different development stages during their differentiation process or during EHT formation is also of interest. In a proof of principal study, we used our confocal trapping setup to measure the force production of car-

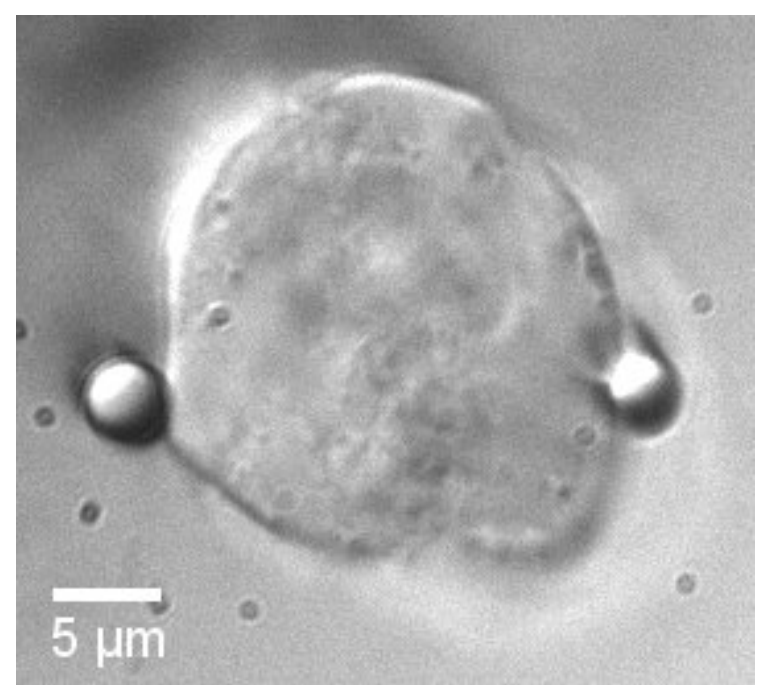

Figure 5.11: Cardiomyocyte cell suspended between two optically trapped $4 \mu \mathrm{m}$ fibronectin coated polystyrene beads. This cell was beating while it was trapped. 
diomyocytes. Cardiomyocytes were differentiated from ihPSCs and cultured in the Zimmermann lab. They were transported to our lab in the same conditions as the primary fibroblast cells. We detached the cells from the surface using an accutase based protocol from the Zimmermann lab (see A.5) and transferred them to the sample solution used for fibroblast experiments: $\mathrm{CO}_{2}$-independent medium supplemented with $10 \%$ FBS. The experiments were done at room temperature. Figure 5.11 shows a cardiomyocyte suspended between two optically trapped beads.
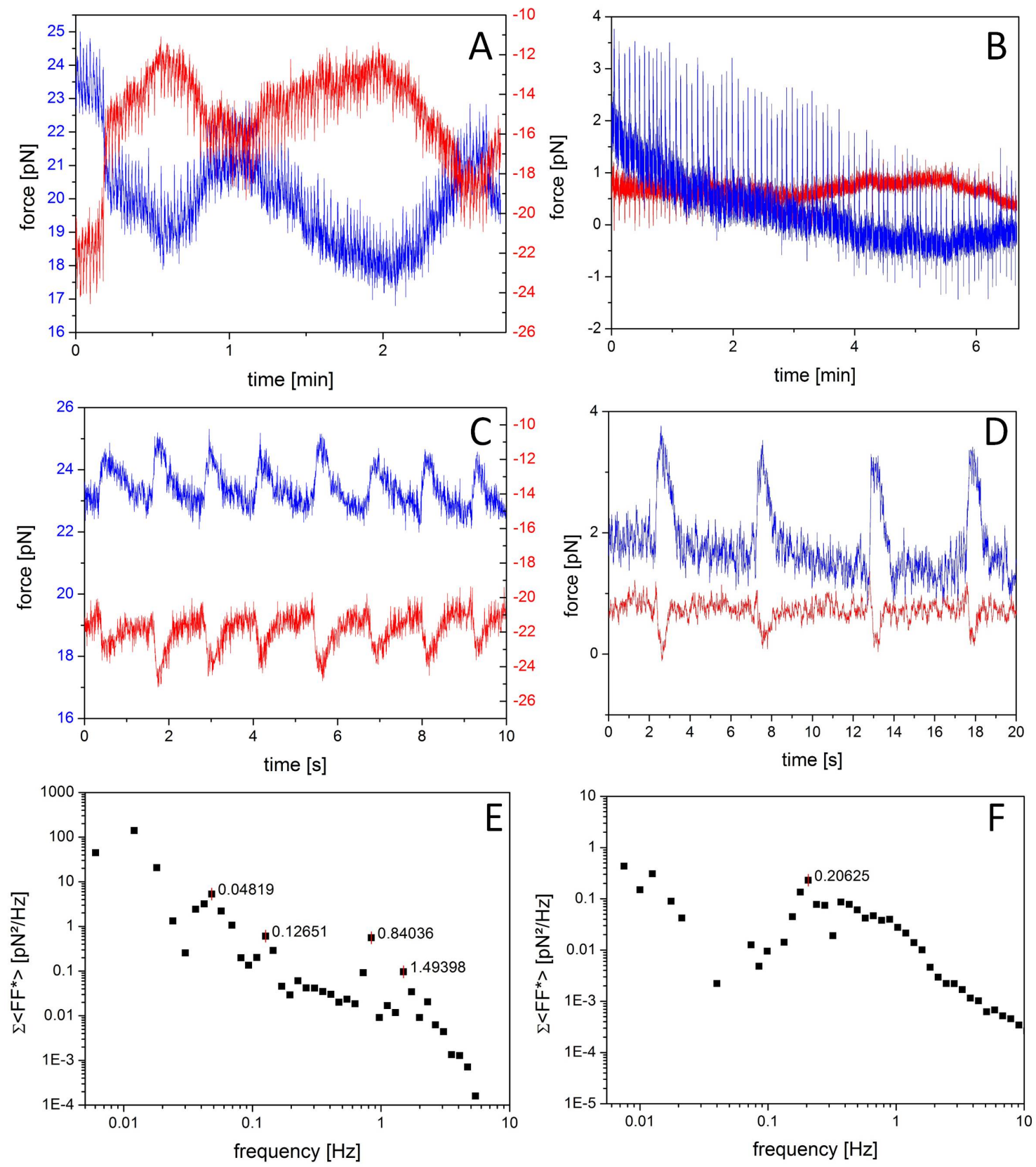

Figure 5.12: Force curves of two attached beads of two beating cardiomyocytes ( $A$ and $B$ ). Zoom-in from $A$ and $B$ to visualize the beating motion of the cells ( $C$ and $D)$. Cross spectra of the two full force measurements with peaks in $\mathrm{Hz}$ that are due to the beating motion ( $\mathrm{E}$ and $\mathrm{F}$ ). Left, blue axis for blue force curve and right, red axis for red force curve (A and $C$ ).

We were able to record the beating motion of two cells in our experimental setup. Figure 5.12 shows force traces of the two attached beads, a zoom-in to visualize the 
beating motion and the correlated force spectrum of the long term measurement. The force production of the cardiomyocytes during their beating motion is around $2 \mathrm{pN}$. Beating frequency of the first cell is around $1 \mathrm{~Hz}$ which is close to their physiological frequency in tissue, whereas that the second cell is around $0.2 \mathrm{~Hz}$ which might be a temperature effect. In the long term measurement of cell one, slow fluctuations similar to the ones that we saw in fibroblasts are visible while the force curve of cell two is rather flat besides the beating motion.

\section{\begin{tabular}{l|l} 
Discussion & 5.4
\end{tabular}}

Engineered heart muscle is composed of different cell types and extracellular matrix, all of them are mechanically connected. One key player besides the cardiomyocytes that generate the beating motion are cardiac fibroblasts. Our experiments showed that also in human cardiac fibroblasts the acto-myosin network plays a major role in the active and passive mechanical properties of the cells. The effect is even more pronounced as in the NIH 3T3 fibroblast cell line with a huge drop in stress fluctuations in CFB after myosin II inhibition (10x lower in NIH 3T3 FB compared to 60x lower in CFB). However, the response of the spring constant to blebbistatin treatment was comparable. Human foreskin fibroblasts and both hiPSC-derived fibroblasts had comparable spring constants to the CFB. The mean spring constant of GFB showed that gingival fibroblasts are roughly half as stiff as cardiac or skin fibroblasts although the difference was not significant due to the limited sample size. If it turns out in future experiments that gingival fibroblasts are significantly softer than other fibroblast cells, together with the fact that they do not adhere as strongly as skin fibroblasts [129] this might be a reason why gingival tissue does not scar in comparison to other tissue types. Our results showed that fibroblasts cells that were differentiated from hiPSC have comparable spring constants to other primary fibroblasts.

Force fluctuations measurements showed that cardiac fibroblasts generate much higher contractile forces than the other cell types. Although there was no significant difference compared to gingival and hiPSC-derived cells due to the limit sample size, their force production was roughly threefold higher compared to HFFs. An effect that had been seen only after myosin inhibition in NIH 3T3 FB. The reduction of force production after blebbistatin treatment of CFB pronounces the major contribution of NMM II to contractile force generation in CFB. The difference in force generation of $\mathrm{CFB}$ compared to other fibroblast types might be due to adaptation to their native environment being constantly under the influence of high mechanical stresses with forces up to the millinewton regime. If it proves that NM 257 fibroblasts have similar high force production, this could also be an effect of their co-culturing with neighboring cardiomyocytes, making them slowly adapting to higher forces.

We showed that it is possible, to measure the beating motion of cardiomyocytes with our optical trap setup. The force production that we saw is almost two orders of magnitude lower compared to other measurements using traction force microscopy 
where rat myocytes sit on silicone rubber gels [130]. Compared to another study measuring contractile forces of hiPSC-CM on elastic pillars, these difference drops to one order of magnitude [131]. Both of this measurements use a very different geometry and experiments were done at $37^{\circ} \mathrm{C}$. However, it is interesting to explore, differences in the force generation of cardiomyocytes at different stages of the differentiation process. 


\section{Conclusions and outlook}

A great variety of cellular processes are influenced by mechanical signals. Cells use forces to probe their environment mechanically and transmit forces through direct contact to neighboring cells or by exerting forces on the elastic fibers in the extracellular matrix. A lot of research has been done on force generation and force transmission of cells on $2 \mathrm{D}$ substrates. In this rather unphysiological situation for most cell types, cells spread out widely and have a well structured acto-myosin stress fiber network which they use to generate high contractile forces. However, it is unclear if cells form a network of stress fibers in their native 3D environment and if this network would be confined to the actin cortex or could span across the cytoplasm. It is also not clear which structures generate shape changes and force fluctuations of cells in a 3D matrix.

Here, we presented a system, which allows us to measure force generation and elastic response of spherical cells that are suspended between two optically trapped beads. We used a dual optical trap to detect force fluctuations with high spatial and temporal resolution. Our experiments, mimicking a 3D environment for cells, showed that both total cellular force generation and effective cell elasticity of fibroblasts are dominated by the acto-myosin cortical network. The microtubule network did not contribute significantly to both properties as revealed by biochemical perturbation experiments using nocodazole. An explanation for this could be that the depolymerization of microtubules enhances traction forces through other signaling pathways [132]. The third prominent member of the cellular cytoskeleton, intermediate filaments, were not be tested here, but in the future we will use whithaferine A, a chemical that disassembles vimentin intermediate filaments [133]. Although it was shown that vimentin plays not a crucial role in the viscoelastic properties of substrate adherent fibroblasts [134] it might be interesting to check if this holds true for rounded cells. It is interesting to investigate the role of other intermediate filaments, as it was shown recently that keratin has a significant impact on cellular stiffness $[135,136]$. One option to study the impact of other cytoskeletal elements, besides the acto-myosin cortex, on cell elasticity is the use of osmotic pressure to collapse the cortex. In preliminary experiments, we showed that with an increase of external osmotic pressure, rounded up cells got softer compared to control conditions, which is in contrast to findings on adherent cells [78]. Using osmotic pressure is another possibility to study the influence of cellular volume on cell mechanics. Recently, it was found that cells use osmotic pressure to adapt their shape and use cell volume as a regulatory mechanism [16]. 
We built a novel instrument, combining a dual optical trap with confocal microscopy. We showed that we are able to use the optical trap to hold cells suspended between two beads and do confocal recordings of fluorescent molecules at the same time for up to one hour. Preliminary results revealed that the actin cortex is thicker when the cell generates higher force fluctuations. However, further markers need to be found to test the interplay between cellular force generation and morphogenesis of the acto-myosin network. There is no answer yet if stress fibers are able to span through the cytosol of suspended cells. Although most of the cells that we have imaged have no actin structures besides the cortex, some cells had also actin fibers sticking out of the cortex to the cytoplasm or wrapping around the nucleus. Improving the trapping strength using core-shell particles [137] will help to see if formation of actin fibers can be induced at a certain force threshold. In the future, the trapping- and confocal imaging laser have to be decoupled to allow for z-scans of trapped objects. This will allow us to investigate the formation of adhesion clusters of suspended cells. Our preliminary results showed that clusters of vinculin form at adhesion sites close to the bead. In order to image such details with even greater resolution, the setup is being equipped with a STED system at the moment.

We explored the mechanical properties of primary fibroblasts and induced pluripotent stem cell derived fibroblasts that are used in engineered heart muscle. We showed that cardiac fibroblasts generate much higher force fluctuations than skin or gingival fibroblasts. This might be explained by the fact that cardiac fibroblasts need to withstand high contractile forces up to millinewtons in their native environment in comparison to other cell types. We showed that we are able to measure force generation of beating cardiomyocytes with our setup. In the future, it should be interesting to investigate how sarcomere structures evolve in cardiomyocytes. For this we need to transfect the cells which is challenging but new technologies using silicon-rhodamine derivatives bound to small molecules might improve the labeling [138]. Furthermore, it should be possible to investigate mechanical properties of cells from engineered heart muscle at different stages of their development process.

In summary, we demonstrate that manipulation of cells in suspended threedimensional geometry provides an alternative technique to probe cell mechanics, complementary to common methods probing adherent cells and amenable to quantitative modeling. 
Appendices 



\section{Biochemical protocols}

\section{Fibronectin coating}

1. wash $1 \mathrm{ml}(50 \mathrm{mg} / \mathrm{ml})$ carboxylated beads $2 \mathrm{x}$ in $10 \mathrm{ml}$ MilliQ water in a centrifuge tube.

2. after second wash resuspend pellet in $10 \mathrm{ml}$ PBS and transfer it to a beaker. Continuously stir the liquid to ensure that the beads are well suspended.

3. while mixing, add $100 \mathrm{mg}$ EDC with constant stirring. Let it react for $15 \mathrm{~min}$ at room temperature.

4. wash $2 \mathrm{x}$ in distilled water and resuspend in $5 \mathrm{ml}$ PBS. Add the calculated amount of fibronectin (saturation at $\mathrm{mg} / \mathrm{ml}, 240 \mu \mathrm{l}$ for $4 \mu \mathrm{m}$ beads). Let it react for 2-4 hours at room temperature with constant stirring.

5. wash and resuspend in $4 \mathrm{ml}$ of quenching solution ( $0.15 \mathrm{~g}$ glycine/50 ml MilliQ $+1 \mathrm{ml}$ of $10 \% \mathrm{BSA}$ ) and mix gently for 30 minutes.

6. wash and resuspend in storage buffer $(0.1 \% \mathrm{BSA}$ in PBS) to desired ( $5 \mathrm{ml})$ storage concentration (often $10 \mathrm{mg} / \mathrm{ml}$ )

7. store at $4^{\circ} \mathrm{C}$ until used.

\section{Neutravidin coating}

Biotinylation of beads

1. wash $50 \mu \mathrm{l}$ bead solution in $950 \mu$ l phosphate buffer

2. add $100 \mu \mathrm{l} \mathrm{EDC}(75 \mathrm{mg} / \mathrm{ml})$

3. add $100 \mu \mathrm{l}$ Biotin-XX, SE $(1 \mathrm{mg} / \mathrm{ml})$

4. incubate for $1 \mathrm{~h}$ at room temperature while shaking 
5. wash $2 \mathrm{x}$ in $1 \mathrm{ml}$ phosphate buffer

6. store at $4^{\circ} \mathrm{C}$ until use

Coat with neutravidin freshly before every use

1. dilute $25 \mu$ l biotinylated beads in $55 \mu$ l phosphate buffer

2. add $10 \mu$ l glycine $(1 \mathrm{M})$

3. add $10 \mu \mathrm{l}$ neutravidin $(5 \mathrm{mg} / \mathrm{ml})$

4. incubate for $1 \mathrm{~h}$ at room temperature while shaking

5. wash $2 \mathrm{x}$ in phosphate buffer

6. store at $4^{\circ} \mathrm{C}$ until use

\section{A.2 Preparation of vesicles}

GUV preparationn by electroswelling

1. prepare lipid solution of 95\% DOPC and 5\% DOPE-Biotinyl in chloroforme

2. put $25 \mu \mathrm{l}$ of lipid solution on two ITO-coated glas slides $(0.5-1.5 \mathrm{mg} / \mathrm{ml})$

3. dry glasslides in exikkator over $\mathrm{NaCl}$-powder over night

4. put a copper strip on each of the glasslides

5. combine the glasslides with a silicon spacer and clammbs to a chamber

6. add desired buffer (Succrose) into chamber without any air bubbles

7. apply a sinusoidal voltage to the copper bands $(12 \mathrm{~Hz}$, increase from $0.05 \mathrm{~V}$ to $1.6 \mathrm{~V}$ within $30 \mathrm{~min}$ )

8. let it react for $3 \mathrm{~h}$

9. apply a square voltage signal $(5 \mathrm{~Hz}, 1.6 \mathrm{~V})$

10. store the vesicles in storage buffer (glucose) for up to one week at $4^{\circ} \mathrm{C}$

11. diameter of vesicles roughly between 10-60 $\mu \mathrm{m}$ 


\section{DDS coating of coverslips}

$\mathrm{KOH}$ cleaning of coverslips

1. put coverslips in a teflon holder in a glass box.

2. add $6 \mathrm{~g}$ of $\mathrm{KOH}$ pellet and dissolve it with a few $\mathrm{ml}$ of MilliQ

3. fill the glass box with EtOH so that the coverslips are fully covered

4. sonicate for $5 \mathrm{~min}$

5. discard KOH solution and sonicate the coverslips 2x in MilliQ

DDS cotaing

1. put the $\mathrm{KOH}$ cleaned coverslips in a glass petri dish with glass beads

2. cover the coverslips with silanization solution I (DDS in heptane)

3. incubate for $10-20 \mathrm{~min}$

4. rinse coverslips in heptane

5. sonicate the coverslips in MilliQ

6. check if coverslips are hydrophobic and store them until usage

1. wash with PBS

2. add $2 \mathrm{ml}$ trypsin $(0.25 \%$ trypsin $+0.1 \%$ EDTA in PBS)

3. incubate for $5 \mathrm{~min}$ at $37^{\circ} \mathrm{C}$

4. add $10 \mathrm{ml}$ culture medium (DMEM+10\% FBS $+1 \%$ penicillin/streptomycin)

5. spin down

6. aspirate the supernatant and resuspend cells in appropriate medium

7. usually 1:10 split for new culture flask 


\section{A.4.2 freeze cells}

1. trypsinize as described

2. resuspend in freezing medium (DMEM $+20 \% \mathrm{FBS}+10 \% \mathrm{DMSO})$

3. fill solution in cryo tubes and store in isopropanol container over night

4. transfer cryotubes to liquid nitrogen

\section{A.4.3 thaw cells}

1. warm cryotube in water bath

2. add $10 \mathrm{ml}$ of culture medium

3. spin down

4. aspirate the supernatant and resuspend cells in appropriate medium

\section{A.5 Detachment of cardiomyocytes}

1. wash cells twice with room temperature PBS

2. add $0.1 \mathrm{ml} / \mathrm{cm}^{2}$ room temperature digestion medium

3. incubate for 10-12 min at room temperature

4. the digestion with treefold amount of serum free medium (SFM) with $5 \mu \mathrm{M}$ Rock inhibitor (not for force measurements), carefully titurate to make single cell suspension and transfer the cells to falcon tube

5. count

6. spin down

7. aspirate the supernatant and resuspend the cells in appropriate medium for downstream application 
store in $10 \mathrm{ml}$ aliquots at $-20^{\circ} \mathrm{C}$, warm to room temperature before use for $100 \mathrm{ml}$ :

1. $97 \mathrm{ml}$ accutase solution (SCR005, Millipore or A11105-01, Gibco)

2. $1 \mathrm{ml} \mathrm{2.5 \%}$ trypsin (final concentration $0.025 \%$ trypsin) (2.5\%, 15090-046, Gibco)

3. 2 ml DNase1 stock (final $20 \mu \mathrm{g} / \mathrm{ml}$ DNase1) (260913, Calbiochem)

\section{serum free medium with Rock inhibitor}

store at $4{ }^{\circ} \mathrm{C}$, warm to room temperature before use

1. RPMI 1640 with glutamax (61870-010, Invitrogen)

2. $1 \%$ of $100 \mathrm{x}$ sodium pyruvate (11360, Invitrogen)

3. $1 \%$ of $100 \mathrm{x}$ penicillin/streptomycin (15140, Invitrogen)

4. 2\% B27 supplement (17504-044, Invitrogen)

5. $200 \mu \mathrm{M}$ ASC (30 mg/500 ml) (A8960-5g, Sigma)

6. 5 $\mu \mathrm{M}$ Rock inhibitor (Y27632, 04-0012-10, Sigma)

\section{Procedure for extracellular microrheology}

Check the setup before preparation and heat the stage to $37^{\circ} \mathrm{C}$ if necessary

1. Create a flow chamber by putting a DDS coated coverslip with doublestick tape on a glasslide

2. infuse $1 \%$ pluronic solution into chamber and let it sit for at least $5 \mathrm{~min}$

3. replace the pluronic solution with $10 \mathrm{x}$ diluted pluronic solution in $\mathrm{CO}_{2}$ independent medium

4. take $900 \mu \mathrm{l} \mathrm{CO}_{2}$ independent medium and add $5 \mu$ bead solution

5. add $100 \mu \mathrm{l}$ of cell solution and $50 \mu \mathrm{l}$ pluronic solution 
6. replace diluted pluronic solution in chamber with the measurement solution

7. check population of beads and cells and adjust accordingly if necessary

8. if desired, seal the chamber with vacuum grease (it will dry out quickly at $37^{\circ}$ C) 


\section{List of references}

[1] C.-M. Lo, H.-B. Wang, M. Dembo, and Y.-l. Wang, "Cell movement is guided by the rigidity of the substrate," Biophysical journal, vol. 79, no. 1, pp. 144$152,2000$.

[2] P. C. Georges, W. J. Miller, D. F. Meaney, E. S. Sawyer, and P. A. Janmey, "Matrices with compliance comparable to that of brain tissue select neuronal over glial growth in mixed cortical cultures," Biophysical journal, vol. 90, no. 8, pp. 3012-3018, 2006.

[3] A. J. Engler, S. Sen, H. L. Sweeney, and D. E. Discher, "Matrix elasticity directs stem cell lineage specification.," Cell, vol. 126, pp. 677-689, Aug 2006.

[4] P. A. Janmey and D. A. Weitz, "Dealing with mechanics: mechanisms of force transduction in cells," Trends in biochemical sciences, vol. 29, no. 7, pp. 364$370,2004$.

[5] A. Bausch and K. Kroy, "A bottom-up approach to cell mechanics," Nature physics, vol. 2, no. 4, pp. 231-238, 2006.

[6] A. W. Orr, B. P. Helmke, B. R. Blackman, and M. A. Schwartz, "Mechanisms of mechanotransduction," Developmental cell, vol. 10, no. 1, pp. 11-20, 2006.

[7] B. Geiger and A. Bershadsky, "Exploring the neighborhood: adhesion-coupled cell mechanosensors," Cell, vol. 110, no. 2, pp. 139-142, 2002.

[8] R. Levayer and T. Lecuit, "Biomechanical regulation of contractility: spatial control and dynamics.," Trends Cell Biol, vol. 22, pp. 61-81, Feb 2012.

[9] S. Pellegrin and H. Mellor, "Actin stress fibres," Journal of cell science, vol. 120, no. 20, pp. 3491-3499, 2007.

[10] M. Vicente-Manzanares, X. Ma, R. S. Adelstein, and A. R. Horwitz, "Nonmuscle myosin ii takes centre stage in cell adhesion and migration," Nature reviews Molecular cell biology, vol. 10, no. 11, pp. 778-790, 2009.

[11] B. D. Hoffman and J. C. Crocker, "Cell mechanics: dissecting the physical responses of cells to force.," Annu Rev Biomed Eng, vol. 11, pp. 259-288, 2009. 
[12] M. Dembo and Y.-L. Wang, "Stresses at the cell-to-substrate interface during locomotion of fibroblasts," Biophysical journal, vol. 76, no. 4, pp. 2307-2316, 1999.

[13] N. Q. Balaban, U. S. Schwarz, D. Riveline, P. Goichberg, G. Tzur, I. Sabanay, D. Mahalu, S. Safran, A. Bershadsky, L. Addadi, et al., "Force and focal adhesion assembly: a close relationship studied using elastic micropatterned substrates," Nature cell biology, vol. 3, no. 5, pp. 466-472, 2001.

[14] B. Sabass, M. L. Gardel, C. M. Waterman, and U. S. Schwarz, "High resolution traction force microscopy based on experimental and computational advances," Biophysical Journal, vol. 94, no. 1, pp. 207-220, 2008.

[15] J. L. Tan, J. Tien, D. M. Pirone, D. S. Gray, K. Bhadriraju, and C. S. Chen, "Cells lying on a bed of microneedles: an approach to isolate mechanical force," Proceedings of the National Academy of Sciences, vol. 100, no. 4, pp. 14841489, 2003.

[16] M. P. Stewart, J. Helenius, Y. Toyoda, S. P. Ramanathan, D. J. Muller, and A. A. Hyman, "Hydrostatic pressure and the actomyosin cortex drive mitotic cell rounding.," Nature, vol. 469, pp. 226-230, Jan 2011.

[17] G. Salbreux, G. Charras, and E. Paluch, "Actin cortex mechanics and cellular morphogenesis," Trends in cell biology, vol. 22, no. 10, pp. 536-545, 2012.

[18] L. Chang and R. D. Goldman, "Intermediate filaments mediate cytoskeletal crosstalk," Nature Reviews Molecular Cell Biology, vol. 5, no. 8, pp. 601-613, 2004 .

[19] O. C. Rodriguez, A. W. Schaefer, C. A. Mandato, P. Forscher, W. M. Bement, and C. M. Waterman-Storer, "Conserved microtubule-actin interactions in cell movement and morphogenesis," Nature cell biology, vol. 5, no. 7, pp. 599-609, 2003 .

[20] A. Ashkin, "Acceleration and trapping of particles by radiation pressure," Physical Review Letters, vol. 24, pp. 156-159, 1970.

[21] A. Ashkin and J. M. Dziedzic, "Optical levitation by radiation pressure," Applied Physics Letters, vol. 19, no. 8, pp. 283-285, 1971.

[22] A. Ashkin, J. M. Dziedzic, J. E. Bjorkholm, and S. Chu, "Observation of a single-beam gradient force optical trap for dielectric particles.," Opt Lett, vol. 11, p. 288, May 1986.

[23] A. Ashkin, "Forces of a single-beam gradient laser trap on a dielectric sphere in the ray optics regime.," Biophys J, vol. 61, pp. 569-582, Feb 1992.

[24] N. Simpson, D. McGloin, K. Dholakia, L. Allen, and M. Padgett, "Optical tweezers with increased axial trapping efficiency," Journal of Modern Optics, vol. 45, no. 9, pp. 1943-1949, 1998. 
[25] A. Sischka, C. Kleimann, W. Hachmann, M. M. Schäfer, I. Seuffert, K. Tönsing, and D. Anselmetti, "Single beam optical tweezers setup with backscattered light detection for three-dimensional measurements on dna and nanopores," Review of Scientific Instruments, vol. 79, no. 6, p. 063702, 2008.

[26] P. M. Neto and H. Nussenzveig, "Theory of optical tweezers," EPL (Europhysics Letters), vol. 50, no. 5, p. 702, 2000.

[27] A. Rohrbach and E. H. Stelzer, "Three-dimensional position detection of optically trapped dielectric particles," Journal of applied physics, vol. 91, no. 8, pp. 5474-5488, 2002.

[28] A. Mazolli, P. M. Neto, and H. Nussenzveig, "Theory of trapping forces in optical tweezers," Proceedings of the Royal Society of London. Series A: Mathematical, Physical and Engineering Sciences, vol. 459, no. 2040, pp. 3021-3041, 2003.

[29] M. Mahamdeh, C. Pérez Campos, and E. Schäffer, "Under-filling trapping objectives optimizes the use of the available laser power in optical tweezers," Optics express, vol. 19, no. 12, pp. 11759-11768, 2011.

[30] F. Gittes and C. F. Schmidt, "Interference model for back-focal-plane displacement detection in optical tweezers.," Opt Lett, vol. 23, pp. 7-9, Jan 1998.

[31] A. Pralle, M. Prummer, E. L. Florin, E. H. Stelzer, and J. K. Hörber, "Threedimensional high-resolution particle tracking for optical tweezers by forward scattered light.," Microsc Res Tech, vol. 44, pp. 378-386, Mar 1999.

[32] W. H. Press, Numerical recipes 3rd edition: The art of scientific computing. Cambridge university press, 2007.

[33] F. Gittes and C. F. Schmidt, "Signals and noise in micromechanical measurements.," Methods Cell Biol, vol. 55, pp. 129-156, 1998.

[34] J. van Mameren, "Single molecule mechanics of biopolymers: An optical tweezers study," Master's thesis, Vrije Universiteit, Amsterdam, 2002.

[35] M. Atakhorrami, K. M. Addas, and C. F. Schmidt, "Twin optical traps for two-particle cross-correlation measurements: eliminating cross-talk.," Rev Sci Instrum, vol. 79, p. 043103, Apr 2008.

[36] C. Pieper, "Techniken zur untersuchung einzelner nicht prozessiver motormoleküle mit optischen fallen," diplomarbeit, Georg-August-Universität Göttingen, 2009.

[37] J. Bechhoefer, "Feedback for physicists: A tutorial essay on control," Reviews of Modern Physics, vol. 77, no. 3, p. 783, 2005.

[38] M. J. Lang, C. L. Asbury, J. W. Shaevitz, and S. M. Block, "An automated two-dimensional optical force clamp for single molecule studies," Biophysical journal, vol. 83, no. 1, pp. 491-501, 2002. 
[39] A. E. Wallin, H. Ojala, G. Ziedaite, and E. Hæggström, "Dual-trap optical tweezers with real-time force clamp control," Review of Scientific Instruments, vol. 82, no. 8, p. 083102, 2011.

[40] D. Mizuno, R. Bacabac, C. Tardin, D. Head, and C. F. Schmidt, "Highresolution probing of cellular force transmission.," Phys Rev Lett, vol. 102, p. 168102, Apr 2009.

[41] D. Mizuno, D. A. Head, F. C. MacKintosh, and C. F. Schmidt, "Active and passive microrheology in equilibrium and nonequilibrium systems," Macromolecules, vol. 41, no. 19, pp. 7194-7202, 2008.

[42] F. Zörgiebel, "Viscoelasticity of bioploymer solutions and the mechanical function of topoisomerases investigated with micromechanical trapping techniques," diplomarbeit, Georg-August-Universität Göttingen, 2009.

[43] J. Riedl, A. H. Crevenna, K. Kessenbrock, J. H. Yu, D. Neukirchen, M. Bista, F. Bradke, D. Jenne, T. A. Holak, Z. Werb, et al., "Lifeact: a versatile marker to visualize f-actin," Nature methods, vol. 5, no. 7, pp. 605-607, 2008.

[44] Q. Wei and R. S. Adelstein, "Conditional expression of a truncated fragment of nonmuscle myosin ii-a alters cell shape but not cytokinesis in hela cells.," Mol Biol Cell, vol. 11, pp. 3617-3627, Oct 2000.

[45] E. Helfer, S. Harlepp, L. Bourdieu, J. Robert, F. C. MacKintosh, and D. Chatenay, "Viscoelastic properties of actin-coated membranes.," Phys Rev E Stat Nonlin Soft Matter Phys, vol. 63, p. 021904, Feb 2001.

[46] V. Vogel and M. Sheetz, "Local force and geometry sensing regulate cell functions.," Nat Rev Mol Cell Biol, vol. 7, pp. 265-275, Apr 2006.

[47] D. A. Fletcher and P. L. Geissler, "Active biological materials.," Annu Rev Phys Chem, vol. 60, pp. 469-486, 2009.

[48] A. Buxboim, I. L. Ivanovska, and D. E. Discher, "Matrix elasticity, cytoskeletal forces and physics of the nucleus: how deeply do cells 'feel' outside and in?," J Cell Sci, vol. 123, pp. 297-308, Feb 2010.

[49] D. E. Discher, P. Janmey, and Y.-L. Wang, "Tissue cells feel and respond to the stiffness of their substrate.," Science, vol. 310, pp. 1139-1143, Nov 2005.

[50] T. Yeung, P. C. Georges, L. A. Flanagan, B. Marg, M. Ortiz, M. Funaki, N. Zahir, W. Ming, V. Weaver, and P. A. Janmey, "Effects of substrate stiffness on cell morphology, cytoskeletal structure, and adhesion.," Cell Motil Cytoskeleton, vol. 60, pp. 24-34, Jan 2005.

[51] A. Zemel, F. Rehfeldt, A. E. X. Brown, D. E. Discher, and S. A. Safran, "Optimal matrix rigidity for stress fiber polarization in stem cells.," Nat Phys, vol. 6, pp. 468-473, Jun 2010.

[52] A. W. C. Lau, B. D. Hoffman, A. Davies, J. C. Crocker, and T. C. Lubensky, "Microrheology, stress fluctuations, and active behavior of living cells.," Phys Rev Lett, vol. 91, p. 198101, Nov 2003. 
[53] A. Mogilner and G. Oster, "Polymer motors: pushing out the front and pulling up the back.," Curr Biol, vol. 13, pp. R721-R733, Sep 2003.

[54] L.-L. Pontani, J. van der Gucht, G. Salbreux, J. Heuvingh, J.-F. Joanny, and C. Sykes, "Reconstitution of an actin cortex inside a liposome.," Biophys J, vol. 96, pp. 192-198, Jan 2009.

[55] F. C. Mackintosh and C. F. Schmidt, "Active cellular materials.," Curr Opin Cell Biol, vol. 22, pp. 29-35, Feb 2010.

[56] D. Mizuno, C. Tardin, C. F. Schmidt, and F. C. Mackintosh, "Nonequilibrium mechanics of active cytoskeletal networks.," Science, vol. 315, pp. 370-373, Jan 2007.

[57] U. S. Schwarz and M. L. Gardel, "United we stand: integrating the actin cytoskeleton and cell-matrix adhesions in cellular mechanotransduction.," $J$ Cell Sci, vol. 125, pp. 3051-3060, Jul 2012.

[58] J. Guck, R. Ananthakrishnan, H. Mahmood, T. J. Moon, C. C. Cunningham, and J. Käs, "The optical stretcher: a novel laser tool to micromanipulate cells.," Biophys J, vol. 81, pp. 767-784, Aug 2001.

[59] J. Guck, S. Schinkinger, B. Lincoln, F. Wottawah, S. Ebert, M. Romeyke, D. Lenz, H. M. Erickson, R. Ananthakrishnan, D. Mitchell, J. Käs, S. Ulvick, and C. Bilby, "Optical deformability as an inherent cell marker for testing malignant transformation and metastatic competence.," Biophys J, vol. 88, pp. 3689-3698, May 2005.

[60] F. Rehfeldt, A. E. Brown, M. Raab, S. Cai, A. L. Zajac, A. Zemel, and D. E. Discher, "Hyaluronic acid matrices show matrix stiffness in $2 \mathrm{~d}$ and $3 \mathrm{~d}$ dictates cytoskeletal order and myosin-ii phosphorylation within stem cells," Integrative biology, vol. 4, no. 4, pp. 422-430, 2012.

[61] G. T. Charras, J. C. Yarrow, M. A. Horton, L. Mahadevan, and T. J. Mitchison, "Non-equilibration of hydrostatic pressure in blebbing cells.," Nature, vol. 435, pp. 365-369, May 2005.

[62] R. Ananthakrishnan, J. Guck, F. Wottawah, S. Schinkinger, B. Lincoln, M. Romeyke, T. Moon, and J. Käs, "Quantifying the contribution of actin networks to the elastic strength of fibroblasts.," J Theor Biol, vol. 242, pp. 502516, Sep 2006.

[63] J. Dai, M. P. Sheetz, X. Wan, and C. E. Morris, "Membrane tension in swelling and shrinking molluscan neurons.," J Neurosci, vol. 18, pp. 6681-6692, Sep 1998.

[64] H. Jiang and S. X. Sun, "Cellular pressure and volume regulation and implications for cell mechanics.," Biophys J, vol. 105, pp. 609-619, Aug 2013.

[65] D. Raucher and M. P. Sheetz, "Characteristics of a membrane reservoir buffering membrane tension.," Biophys J, vol. 77, pp. 1992-2002, Oct 1999. 
[66] L. L. Norman, J. Brugués, J. Brugés, K. Sengupta, P. Sens, and H. ArandaEspinoza, "Cell blebbing and membrane area homeostasis in spreading and retracting cells.," Biophys J, vol. 99, pp. 1726-1733, Sep 2010.

[67] M. P. Murrell and M. L. Gardel, "F-actin buckling coordinates contractility and severing in a biomimetic actomyosin cortex.," Proc Natl Acad Sci U S A, vol. 109, pp. 20820-20825, Dec 2012.

[68] S. K. Vogel, Z. Petrasek, F. Heinemann, and P. Schwille, "Myosin motors fragment and compact membrane-bound actin filaments.," Elife, vol. 2, p. e00116, 2013.

[69] F. Schlosser, F. Rehfeldt, and C. F. Schmidt, "Force fluctuations in threedimensional suspended fibroblasts.," Philos Trans $R$ Soc Lond B Biol Sci, vol. 370, p. 20140028, Feb 2015.

[70] F. Gittes, B. Mickey, J. Nettleton, and J. Howard, "Flexural rigidity of microtubules and actin filaments measured from thermal fluctuations in shape.," $J$ Cell Biol, vol. 120, pp. 923-934, Feb 1993.

[71] M. Abe, C.-H. Ho, K. E. Kamm, and F. Grinnell, "Different molecular motors mediate platelet-derived growth factor and lysophosphatidic acid-stimulated floating collagen matrix contraction.," J Biol Chem, vol. 278, pp. 47707-47712, Nov 2003.

[72] T. Wakatsuki, R. B. Wysolmerski, and E. L. Elson, "Mechanics of cell spreading: role of myosin ii.," J Cell Sci, vol. 116, pp. 1617-1625, Apr 2003.

[73] A. J. Levine and F. C. MacKintosh, "The mechanics and fluctuation spectrum of active gels.," J Phys Chem B, vol. 113, pp. 3820-3830, Mar 2009.

[74] G. Salbreux, J. F. Joanny, J. Prost, and P. Pullarkat, "Shape oscillations of non-adhering fibroblast cells.," Phys Biol, vol. 4, pp. 268-284, Dec 2007.

[75] S. M. Mijailovich, M. Kojic, M. Zivkovic, B. Fabry, and J. J. Fredberg, "A finite element model of cell deformation during magnetic bead twisting," Journal of Applied Physiology, vol. 93, no. 4, pp. 1429-1436, 2002.

[76] B. Fabry, G. N. Maksym, J. P. Butler, M. Glogauer, D. Navajas, and J. J. Fredberg, "Scaling the microrheology of living cells," Physical review letters, vol. 87 , no. 14, p. 148102, 2001.

[77] C. G. Galbraith, K. M. Yamada, and M. P. Sheetz, "The relationship between force and focal complex development.," J Cell Biol, vol. 159, pp. 695-705, Nov 2002 .

[78] E. Zhou, X. Trepat, C. Park, G. Lenormand, M. Oliver, S. Mijailovich, C. Hardin, D. Weitz, J. Butler, and J. Fredberg, "Universal behavior of the osmotically compressed cell and its analogy to the colloidal glass transition," Proceedings of the National Academy of Sciences, vol. 106, no. 26, pp. 1063210637, 2009. 
[79] S. Nawaz, P. Sánchez, K. Bodensiek, S. Li, M. Simons, and I. A. T. Schaap, "Cell visco-elasticity measured with afm and optical trapping at submicrometer deformations.," PLoS One, vol. 7, no. 9, p. e45297, 2012.

[80] C. Rotsch and M. Radmacher, "Drug-induced changes of cytoskeletal structure and mechanics in fibroblasts: an atomic force microscopy study.," Biophys J, vol. 78, pp. 520-535, Jan 2000.

[81] C. P. Brangwynne, F. C. MacKintosh, S. Kumar, N. A. Geisse, J. Talbot, L. Mahadevan, K. K. Parker, D. E. Ingber, and D. A. Weitz, "Microtubules can bear enhanced compressive loads in living cells because of lateral reinforcement.," J Cell Biol, vol. 173, pp. 733-741, Jun 2006.

[82] J. Dai, H. P. Ting-Beall, R. M. Hochmuth, M. P. Sheetz, and M. A. Titus, "Myosin i contributes to the generation of resting cortical tension.," Biophys J, vol. 77, pp. 1168-1176, Aug 1999.

[83] J. Limouze, A. F. Straight, T. Mitchison, and J. R. Sellers, "Specificity of blebbistatin, an inhibitor of myosin ii.," J Muscle Res Cell Motil, vol. 25, no. 4-5, pp. 337-341, 2004.

[84] F. C. MacKintosh and A. J. Levine, "Nonequilibrium mechanics and dynamics of motor-activated gels," Phys. Rev. Lett., vol. 100, p. 018104, Jan 2008.

[85] C. J. Chan, A. E. Ekpenyong, S. Golfier, W. Li, K. J. Chalut, O. Otto, J. Elgeti, J. Guck, and F. Lautenschläger, "Myosin ii activity softens cells in suspension," Biophysical journal, vol. 108, no. 8, pp. 1856-1869, 2015.

[86] N. Fakhri, A. D. Wessel, C. Willms, M. Pasquali, D. R. Klopfenstein, F. C. MacKintosh, and C. F. Schmidt, "High-resolution mapping of intracellular fluctuations using carbon nanotubes.," Science, vol. 344, pp. 1031-1035, May 2014.

[87] R. Sunyer, X. Trepat, J. J. Fredberg, R. Farré, and D. Navajas, "The temperature dependence of cell mechanics measured by atomic force microscopy.," Phys Biol, vol. 6, no. 2, p. 025009, 2009.

[88] T. Wakatsuki and E. L. Elson, "Reciprocal interactions between cells and extracellular matrix during remodeling of tissue constructs.," Biophys Chem, vol. 100, no. 1-3, pp. 593-605, 2003.

[89] A. Düselder, "Einzelmolekülmessungen des motorproteins eg5kin mit einer optischen falle," diplomarbeit, Georg-August-Universität Göttingen, 2009.

[90] M. Bremerich, High-bandwidth microrheology of cytoskeletal networks. PhD thesis, Georg-August-Universität Göttingen, 2011.

[91] L. Barros, T. Kanaseki, R. Sabirov, S. Morishima, J. Castro, C. Bittner, E. Maeno, Y. Ando-Akatsuka, and Y. Okada, "Apoptotic and necrotic blebs in epithelial cells display similar neck diameters but different kinase dependency," Cell Death ES Differentiation, vol. 10, no. 6, pp. 687-697, 2003. 
[92] M. Sixt, "Cell migration: fibroblasts find a new way to get ahead," The Journal of cell biology, vol. 197, no. 3, pp. 347-349, 2012.

[93] A. G. Clark, K. Dierkes, and E. K. Paluch, "Monitoring actin cortex thickness in live cells," Biophysical journal, vol. 105, no. 3, pp. 570-580, 2013.

[94] R. Zaidel-Bar, S. Itzkovitz, A. Ma'ayan, R. Iyengar, and B. Geiger, "Functional atlas of the integrin adhesome," Nature cell biology, vol. 9, no. 8, pp. 858-867, 2007.

[95] C. Grashoff, B. D. Hoffman, M. D. Brenner, R. Zhou, M. Parsons, M. T. Yang, M. A. McLean, S. G. Sligar, C. S. Chen, T. Ha, et al., "Measuring mechanical tension across vinculin reveals regulation of focal adhesion dynamics," Nature, vol. 466, no. 7303, pp. 263-266, 2010.

[96] I. Heller, G. Sitters, O. D. Broekmans, G. Farge, C. Menges, W. Wende, S. W. Hell, E. J. Peterman, and G. J. Wuite, "Sted nanoscopy combined with optical tweezers reveals protein dynamics on densely covered dna," Nature methods, vol. 10, no. 9, pp. 910-916, 2013.

[97] J. van Mameren, K. C. Vermeulen, F. Gittes, and C. F. Schmidt, "Leveraging single protein polymers to measure flexural rigidity†," The Journal of Physical Chemistry B, vol. 113, no. 12, pp. 3837-3844, 2009.

[98] S. I. Fraley, Y. Feng, R. Krishnamurthy, D.-H. Kim, A. Celedon, G. D. Longmore, and D. Wirtz, "A distinctive role for focal adhesion proteins in threedimensional cell motility," Nature cell biology, vol. 12, no. 6, pp. 598-604, 2010 .

[99] V. L. Roger, A. S. Go, D. M. Lloyd-Jones, E. J. Benjamin, J. D. Berry, W. B. Borden, D. M. Bravata, S. Dai, E. S. Ford, C. S. Fox, et al., "Heart disease and stroke statistics - 2012 update a report from the american heart association," Circulation, vol. 125, no. 1, pp. e2-e220, 2012.

[100] B. G. Bruneau, "The developmental genetics of congenital heart disease," Nature, vol. 451, no. 7181, pp. 943-948, 2008.

[101] S. E. Lipshultz, L. A. Sleeper, J. A. Towbin, A. M. Lowe, E. J. Orav, G. F. Cox, P. R. Lurie, K. L. McCoy, M. A. McDonald, J. E. Messere, et al., "The incidence of pediatric cardiomyopathy in two regions of the united states," New England Journal of Medicine, vol. 348, no. 17, pp. 1647-1655, 2003.

[102] J. Lexchin, "Drug withdrawals from the canadian market for safety reasons, 1963-2004," Canadian Medical Association Journal, vol. 172, no. 6, pp. 765$767,2005$.

[103] M. Didié, P. Christalla, M. Rubart, V. Muppala, S. Döker, B. Unsöld, A. ElArmouche, T. Rau, T. Eschenhagen, A. P. Schwoerer, et al., "Parthenogenetic stem cells for tissue-engineered heart repair," The Journal of clinical investigation, vol. 123, no. 3, p. 1285, 2013. 
[104] W. H. Zimmermann, C. Fink, D. Kralisch, U. Remmers, J. Weil, and T. Eschenhagen, "Three-dimensional engineered heart tissue from neonatal rat cardiac myocytes," Biotechnology and bioengineering, vol. 68, no. 1, pp. 106-114, 2000.

[105] W.-H. Zimmermann and T. Eschenhagen, "Cardiac tissue engineering for replacement therapy," Heart failure reviews, vol. 8, no. 3, pp. 259-269, 2003.

[106] M. S. Kolodney and E. L. Elson, "Correlation of myosin light chain phosphorylation with isometric contraction of fibroblasts.," J Biol Chem, vol. 268, pp. 23850-23855, Nov 1993.

[107] W.-H. Zimmermann, K. Schneiderbanger, P. Schubert, M. Didie, F. Münzel, J. Heubach, S. Kostin, W. Neuhuber, and T. Eschenhagen, "Tissue engineering of a differentiated cardiac muscle construct," Circulation research, vol. 90, no. 2, pp. 223-230, 2002.

[108] B. Chandapillai Karikkineth and W.-H. Zimmermann, "Myocardial tissue engineering and heart muscle repair," Current pharmaceutical biotechnology, vol. 14, no. 1, pp. 4-11, 2013.

[109] J. Yu, M. A. Vodyanik, K. Smuga-Otto, J. Antosiewicz-Bourget, J. L. Frane, S. Tian, J. Nie, G. A. Jonsdottir, V. Ruotti, R. Stewart, et al., "Induced pluripotent stem cell lines derived from human somatic cells," Science, vol. 318, no. 5858, pp. 1917-1920, 2007.

[110] K. Takahashi, K. Tanabe, M. Ohnuki, M. Narita, T. Ichisaka, K. Tomoda, and S. Yamanaka, "Induction of pluripotent stem cells from adult human fibroblasts by defined factors," cell, vol. 131, no. 5, pp. 861-872, 2007.

[111] K. Streckfuss-Bömeke, F. Wolf, A. Azizian, M. Stauske, M. Tiburcy, S. Wagner, D. Hübscher, R. Dressel, S. Chen, J. Jende, et al., "Comparative study of human-induced pluripotent stem cells derived from bone marrow cells, hair keratinocytes, and skin fibroblasts," European heart journal, vol. 34, no. 33, pp. 2618-2629, 2013.

[112] A. Somers, J.-C. Jean, C. A. Sommer, A. Omari, C. C. Ford, J. A. Mills, L. Ying, A. G. Sommer, J. M. Jean, B. W. Smith, et al., "Generation of transgene-free lung disease-specific human induced pluripotent stem cells using a single excisable lentiviral stem cell cassette," Stem cells, vol. 28, no. 10, pp. 1728-1740, 2010.

[113] W.-H. Zimmermann, "Embryonic and embryonic-like stem cells in heart muscle engineering," Journal of molecular and cellular cardiology, vol. 50, no. 2, pp. 320-326, 2011.

[114] B. I. Jugdutt, "Ventricular remodeling after infarction and the extracellular collagen matrix when is enough enough?," Circulation, vol. 108, no. 11, pp. 1395-1403, 2003. 
[115] D. L. Brutsaert, G. W. De Keulenaer, P. Fransen, P. Mohan, G. L. Kaluza, L. J. Andries, J.-L. Rouleau, and S. U. Sys, "The cardiac endothelium: functional morphology, development, and physiology," Progress in cardiovascular diseases, vol. 39, no. 3, pp. 239-262, 1996.

[116] M. Harada, H. Itoh, O. Nakagawa, Y. Ogawa, Y. Miyamoto, K. Kuwahara, E. Ogawa, T. Igaki, J. Yamashita, I. Masuda, et al., "Significance of ventricular myocytes and nonmyocytes interaction during cardiocyte hypertrophy evidence for endothelin-1 as a paracrine hypertrophic factor from cardiac nonmyocytes," Circulation, vol. 96, no. 10, pp. 3737-3744, 1997.

[117] H. Naito, I. Melnychenko, M. Didié, K. Schneiderbanger, P. Schubert, S. Rosenkranz, T. Eschenhagen, and W.-H. Zimmermann, "Optimizing engineered heart tissue for therapeutic applications as surrogate heart muscle," Circulation, vol. 114, no. 1 suppl, pp. I-72, 2006.

[118] G. Kensah, A. R. Lara, J. Dahlmann, R. Zweigerdt, K. Schwanke, J. Hegermann, D. Skvorc, A. Gawol, A. Azizian, S. Wagner, et al., "Murine and human pluripotent stem cell-derived cardiac bodies form contractile myocardial tissue in vitro," European heart journal, p. ehs349, 2012.

[119] A. Lesman, M. Habib, O. Caspi, A. Gepstein, G. Arbel, S. Levenberg, and L. Gepstein, "Transplantation of a tissue-engineered human vascularized cardiac muscle," Tissue Engineering Part A, vol. 16, pp. 115-125, July 2009.

[120] K. Stevens, K. Kreutziger, S. Dupras, F. Korte, M. Regnier, V. Muskheli, M. Nourse, K. Bendixen, H. Reinecke, and C. Murry, "Physiological function and transplantation of scaffold-free and vascularized human cardiac muscle tissue," Proceedings of the National Academy of Sciences, vol. 106, no. 39, pp. 16568-16573, 2009.

[121] I. Banerjee, J. W. Fuseler, R. L. Price, T. K. Borg, and T. A. Baudino, "Determination of cell types and numbers during cardiac development in the neonatal and adult rat and mouse," American Journal of Physiology-Heart and Circulatory Physiology, vol. 293, no. 3, pp. H1883-H1891, 2007.

[122] M. Tiburcy, M. Didié, O. Boy, P. Christalla, S. Döker, H. Naito, B. C. Karikkineth, A. El-Armouche, M. Grimm, M. Nose, et al., "Terminal differentiation, advanced organotypic maturation, and modeling of hypertrophic growth in engineered heart tissue," Circulation research, vol. 109, no. 10, pp. 1105-1114, 2011.

[123] C. A. Souders, S. L. Bowers, and T. A. Baudino, "Cardiac fibroblast the renaissance cell," Circulation research, vol. 105, no. 12, pp. 1164-1176, 2009.

[124] M. Ieda, T. Tsuchihashi, K. N. Ivey, R. S. Ross, T.-T. Hong, R. M. Shaw, and D. Srivastava, "Cardiac fibroblasts regulate myocardial proliferation through $\beta 1$ integrin signaling," Developmental cell, vol. 16, no. 2, pp. 233-244, 2009.

[125] R. Kakkar and R. T. Lee, "Intramyocardial fibroblast myocyte communication," Circulation Research, vol. 106, no. 1, pp. 47-57, 2010. 
[126] R. P. Butt and J. E. Bishop, "Mechanical load enhances the stimulatory effect of serum growth factors on cardiac fibroblast procollagen synthesis," Journal of molecular and cellular cardiology, vol. 29, no. 4, pp. 1141-1151, 1997.

[127] W. Carver, M. Nagpal, M. Nachtigal, T. Borg, and L. Terracio, "Collagen expression in mechanically stimulated cardiac fibroblasts.," Circulation research, vol. 69, no. 1, pp. 116-122, 1991.

[128] A. H. Hsieh, C. M.-H. Tsai, Q.-J. Ma, T. Lin, A. J. Banes, F. J. Villarreal, W. H. Akeson, and K.-L. Paul Sung, "Time-dependent increases in type-iii collagen gene expression in medial collateral ligament fibroblasts under cyclic strains," Journal of Orthopaedic Research, vol. 18, no. 2, pp. 220-227, 2000.

[129] F. Guo, D. E. Carter, A. Mukhopadhyay, and A. Leask, "Gingival fibroblasts display reduced adhesion and spreading on extracellular matrix: a possible basis for scarless tissue repair?," PloS one, vol. 6, no. 11, p. e27097, 2011.

[130] N. Hersch, B. Wolters, G. Dreissen, R. Springer, N. Kirchgeßner, R. Merkel, and B. Hoffmann, "The constant beat: cardiomyocytes adapt their forces by equal contraction upon environmental stiffening," Biology open, vol. 2, no. 3, pp. 351-361, 2013.

[131] M. L. Rodriguez, B. T. Graham, L. M. Pabon, S. J. Han, C. E. Murry, and N. J. Sniadecki, "Measuring the contractile forces of human induced pluripotent stem cell-derived cardiomyocytes with arrays of microposts," Journal of biomechanical engineering, vol. 136, no. 5, p. 051005, 2014.

[132] A. Rape, W.-h. Guo, and Y.-l. Wang, "Microtubule depolymerization induces traction force increase through two distinct pathways," Journal of cell science, vol. 124, no. 24, pp. 4233-4240, 2011.

[133] P. Bargagna-Mohan, A. Hamza, Y.-e. Kim, Y. K. A. Ho, N. Mor-Vaknin, N. Wendschlag, J. Liu, R. M. Evans, D. M. Markovitz, C.-G. Zhan, et al., "The tumor inhibitor and antiangiogenic agent withaferin a targets the intermediate filament protein vimentin," Chemistry \& biology, vol. 14, no. 6, pp. 623-634, 2007.

[134] M. Guo, A. J. Ehrlicher, S. Mahammad, H. Fabich, M. H. Jensen, J. R. Moore, J. J. Fredberg, R. D. Goldman, and D. A. Weitz, "The role of vimentin intermediate filaments in cortical and cytoplasmic mechanics," Biophysical journal, vol. 105, no. 7, pp. 1562-1568, 2013.

[135] J. Kayser, M. Haslbeck, L. Dempfle, M. Krause, C. Grashoff, J. Buchner, H. Herrmann, and A. R. Bausch, "The small heat shock protein hsp27 affects assembly dynamics and structure of keratin intermediate filament networks," Biophysical journal, vol. 105, no. 8, pp. 1778-1785, 2013.

[136] K. Seltmann, A. W. Fritsch, J. A. Käs, and T. M. Magin, "Keratins significantly contribute to cell stiffness and impact invasive behavior," Proceedings of the National Academy of Sciences, vol. 110, no. 46, pp. 18507-18512, 2013. 
[137] A. Jannasch, A. F. Demirörs, P. D. van Oostrum, A. van Blaaderen, and E. Schäffer, "Nanonewton optical force trap employing anti-reflection coated, high-refractive-index titania microspheres," Nature Photonics, vol. 6, no. 7, pp. 469-473, 2012.

[138] G. Lukinavičius, L. Reymond, E. D'Este, A. Masharina, F. Göttfert, H. Ta, A. Güther, M. Fournier, S. Rizzo, H. Waldmann, et al., "Fluorogenic probes for live-cell imaging of the cytoskeleton," Nature methods, vol. 11, no. 7, pp. 731733, 2014. 


\section{Danksagung/Acknowledgments}

$\mathrm{Zu}$ allererst möchte ich meinen beiden Betreuern Dr. Florian Rehfeldt und Prof. Christoph Schmidt danken. Sie haben es mir ermöglicht auf einem für mich außerordentlich spannenden Gebiet meine Arbeit anzufertigen. Darüber hinaus haben Sie sich stets Zeit genommen um mir bei meinen Fragen und Problemen zu Helfen und mit neuen Ideen einen anderen Blickwinkel auf die Probleme zu geben.

Bedanken möchte ich mich auch bei den weiteren Mitgliedern meines Betreuungsausschusses, Prof. Sarah Köster, insbesondere auch für die Übernahme der Zweitkorrektur, und bei Prof. Andreas Janshoff für die Begleitung während der Arbeit und guten Hinweisen in den Kommitteetreffen.

Danke auch an Susanne Schlick, die die Kardiomyocyten und primären Fibroblasten zur Verfügung gestellt und alle meine Fragen dazu geduldig beantwortet hat.

Ein großer Dank auch an die "TAs"Tanja Gall, Charlotte Willms, Ulrike Schulz und Kerstin von Roden für Hilfe und Unterstützung bei der "Betreuung"der Zellen, zahlreichen Bestellungen und alles andere was mit den Laboren zu tun hatte.

I also like to thank Prof. Daisuke Mizuno who introduced me into the big trap and microrheology and helped me with questions during his stays in Germany. Special thanks go also to André Düselder, Marcel Bremerich, Christoph Pieper und Felix Zörgiebel who worked with me on the trap and helped to solve various big and small problems throughout the time.

A big thank, of course, to all of my former and present collegues at our group in the biophysics institute: Alok, André, Carina, Charlotte, Chris, Christina, Daniel, Dieter, Eugenia, Fabian, Florian, Frederike, Galina, Iwan, Jannes, Kerstin, Marcel, Meenakshi, Miquel, Mitja, Nikta, Paula, Phillip, Samaneh, Susanne, Tanja, Theresa, Til, Ullrich, Ulrike and all I forgot. I enjoyed the motivational support, all the nice lunch time talks and activities in and outside of the lab.

Ein großes Dankeschön auch an alle Leute, die mich durch die gesamte Studienzeit begleitet haben und mit denen ich jede Menge Spaß hatte und habe: André Düselder, Marie Schmidt, Stefan Rudas, Tobias Reusch, Friederike Reusch, Katharina Burmeister, HaJo Helms, Max Gulde, Robert Gröbel, Brita Rohrbeck, Stefan Küchemann und Anke Bludau.

Vielen Dank an Armin Kockel, für unseren gemeinsamen Weg seit Kindestagen an und eine besondere Freundschaft. 
Besonderen Dank an meine Eltern und meine Familie für die ganze Unterstützung in allen erdenklichen Formen über all die Jahre. Ohne Euch wäre das alles nicht möglich gewesen.

Am Ende danke ich Karina, dass Sie immer für mich da ist und ich freue mich auf unsere gemeinsame Zukunft.

\section{Thank you!}




\section{Curriculum Vitae}

\section{Personal data}

Name:

Florian Schlosser

Address:

Kaakweg 3

37077 Göttingen

Germany

E-Mail: $\quad$ florian.schlosser@phys.uni-goettingen.de

Date of birth: $\quad 12 / 17 / 1983$

Nationality: German

\section{Education}

06/2011 - Present

PhD studies in Physics

Georg-August University Göttingen, Germany

Third Institute of Physics - Biophysics

$\mathrm{PhD}$ thesis: "Morphogenesis of the Force-Generating Machinery in Cells"

Supervisors: Prof. Christoph F. Schmidt, and Dr. Florian Rehfeldt

$06 / 2011$

Accepted at the Göttingen Graduate School for Neurosciences, Biophysics, and Molecular Biosciences

within the doctoral program "Physics of Biological and Complex Systems"

10/2004 - 05/2011 Diploma in Physics (equivalent to M.Sc.)

Georg-August University Göttingen, Germany

Third Institute of Physics - Biophysics

Diploma thesis: "Force-Generation and Elasticity of 3T3 Fibroblasts - An Optical Tweezer Study"

Supervisor: Prof. Christoph F. Schmidt

08/2003 - 05/2004 Military service 
08/1996 - 06/2003 Abitur

Hannah-Arendt Gymnasium, Barsinghausen, Germany

\section{Publications}

Phil. Trans. R. Soc. B F. Schlosser, F. Rehfeldt and C.F. Schmidt, "Force fluctuations in three-dimensional suspended fibroblasts", 370, 20140028, Dec 2014.

June 12, 2015 